\title{
Physical Activity and its Association with White Matter Integrity and Cognitive Efficiency in Pediatric-Onset Multiple Sclerosis
}

\author{
Elisea De Somma
}

A Thesis Submitted to the Faculty of Graduate Studies in Partial Fulfillment of the Requirements for the Degree of Master of Arts

\author{
Graduate Program in Clinical Developmental Psychology \\ York University \\ Toronto, Ontario
}

July 2017

CElisea De Somma, 2017 


\begin{abstract}
In healthy children and adolescents, increased white matter integrity is highly correlated with cognitive and language development. Moreover, higher level of physical activity has been shown to confer positive effects on cognition and is associated with increased white matter microstructure in children. Decreased white matter integrity and impaired cognitive efficiency (i.e., speed of information processing) are consistently observed in pediatric-onset multiple sclerosis (MS) compared to healthy controls. However, the association between physical activity, white matter integrity, and cognition has yet to be fully described in this population. To address this gap in the literature, this study aimed to determine whether level of self-reported strenuous physical activity is associated with white matter integrity and cognitive efficiency in pediatriconset MS patients. We examined white matter integrity in 24 pediatric-onset MS patients and 34 age- and sex-matched healthy controls via diffusion tensor imaging (DTI) using tract-based spatial statistics (TBSS). Participants also completed a neuropsychological test battery (from which a cognitive efficiency composite score was derived), and a questionnaire examining selfreported strenuous physical activity. Findings demonstrated that patients had significantly lower white matter integrity relative to healthy controls in the entire white matter skeleton (i.e., across the whole brain) and in 21 of 26 interrogated white matter tracts. Cognitive outcomes did not associate with indices of white matter integrity. Self-reported strenuous physical activity also did not associate with white matter integrity; however, it was positively associated with white matter volume in healthy controls, and trended towards a positive association in patients. The results of this study confirm that DTI parameters are sensitive to disease pathology in pediatric-onset MS. While we did not confirm that white matter integrity was associated with cognitive efficiency, this cohort of patients was relatively cognitively preserved, likely masking any relation. No association with self-reported strenuous physical activity was noted; however further investigation of the positive effects of physical activity on white matter in pediatric-onset MS is warranted, as its role in moderating cognitive functioning in this population remains to be elucidated.
\end{abstract}




\section{Acknowledgements}

To my supervisor, Dr. Christine Till, thank you for your unwavering support throughout my MA study. Thank you for challenging me to think critically about my research, and for encouraging me to venture down new avenues of thought. Our conversations make me more excited about the research I do. Thank you for your continued enthusiasm regarding my work, and for creating a positive work environment that stimulates thoughtful discussion.

To Dr. Jennifer Steeves, thank you for your positive and constructive feedback regarding this thesis. Your input has greatly contributed to how I have conceptualized my research.

To my labmates, thank you for creating a workspace that is fun, supportive, and collaborative. To Rivky, thank you for being a constant source of positive encouragement. To Emily, thank you for being a dear friend, confidant, and for providing me with your insights and guidance.

To Dr. Ritobrato Datta, thank you for your continuous support with my DTI analyses. Without your guidance, I would not have been able to complete this work. Thank you for being a thoughtful teacher, for welcoming my questions, and for the time you spent helping me troubleshoot my analyses.

To the members of my lab who came before me, Nadine and Magdalena, thank you for your major contributions in recruiting and running participants for this study. Your time and effort in designing and facilitating the completion of this study is invaluable.

Thank you to our collaborators at the Montreal Neurological Institute for conducting the structural MRI analyses for this project. To Drs. Brenda Banwell and Ann Yeh, thank you for the time you have taken to provide your feedback regarding my work. Your direction is greatly appreciated and has challenged me to think about my research with new perspectives.

Finally, to my family, thank you for your continued support in my academic endeavours. Thank you for teaching me the value of hard work, but most importantly, thank you for teaching me to have fun along the way, and to enjoy the journey. 
Table of Contents

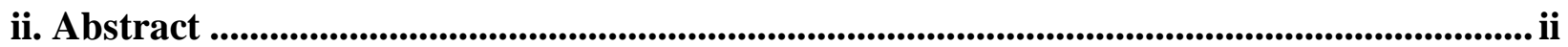

iii. Acknowledgements ...................................................................................................................... iii

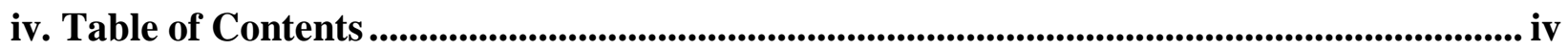

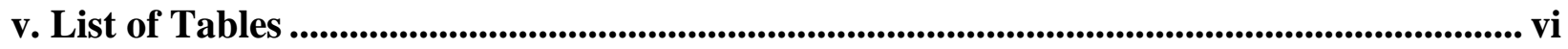

vi. List of Figures..................................................................................................................................... vii

vii. List of Abbreviations .................................................................................................................. viii

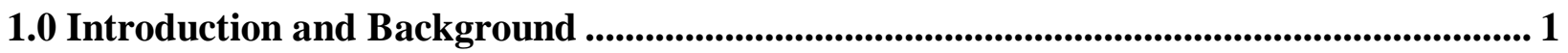

1.1 Overview of Multiple Sclerosis ................................................................................... 1

1.1.1 Diagnosis and Causes .................................................................................................................... 1

1.1.2 Pediatric-Onset Multiple Sclerosis ................................................................................. 2

1.2 Multiple Sclerosis and the Developing Brain ........................................................................ 3

1.3 Cognitive Impairment in Pediatric-Onset MS ............................................................... 5

1.3.1 Progression of Cognitive Deficits ............................................................................................ 5

1.3.2 Cognitive Efficiency in Pediatric-Onset $M S$............................................................... 6

1.4 White Matter Integrity in Pediatric-Onset MS................................................................ 7

1.4.1 Measuring White Matter Integrity: Diffusion Tensor Imaging ................................ 7

1.4.2 White Matter Changes in Pediatric-Onset MS ........................................................... 9

1.4.3 White Matter Damage and Cognitive Efficiency in Pediatric-Onset MS .................. 9

1.5 Physical Activity and Brain Structure and Function .......................................................... 12

1.5.1 Benefits of Physical Activity in Healthy Children and Adolescents ........................ 12

1.5.2 Evidence from Adult MS Research ........................................................................... 13

1.5.3 Preliminary Evidence in Pediatric Onset MS ....................................................... 14

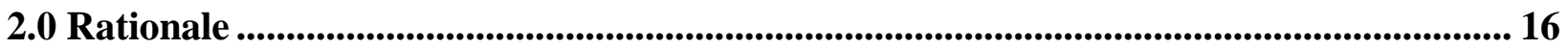

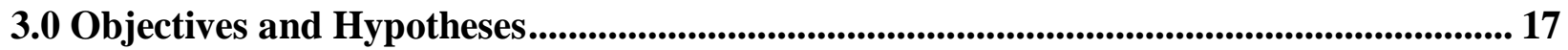

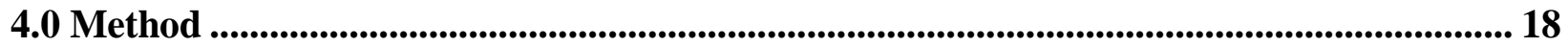

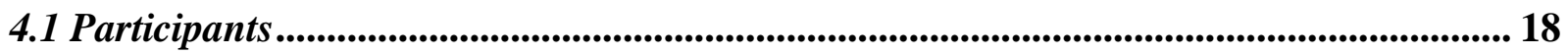

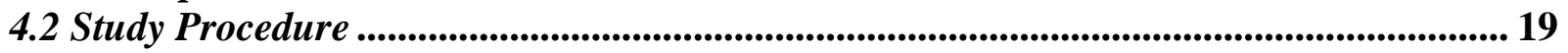

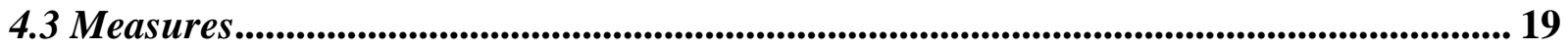

4.3.1 Clinical-Demographic Information .................................................................................. 19

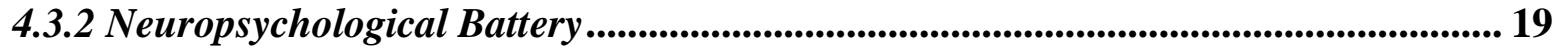

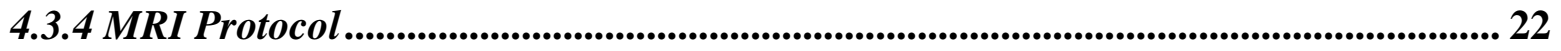

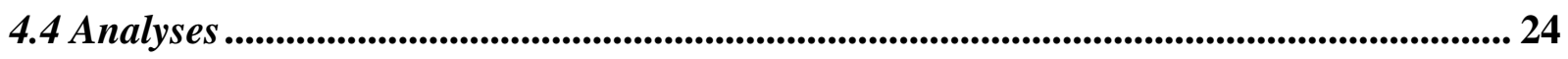

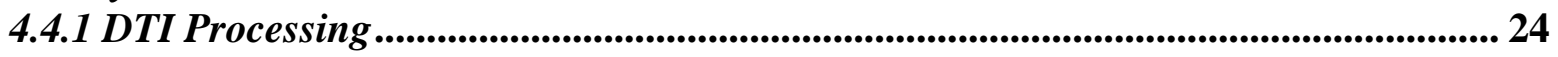

4.4.2 Statistical Analyses .............................................................................................................. 25

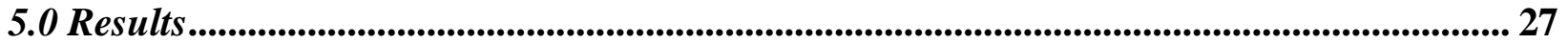

5.1 Sample Characteristics ..................................................................................................... 27

5.2 Diffusion Tensor Imaging (DTI) Results ................................................................... 30

5.3 DTI Correlation Analyses .................................................................................. 33 


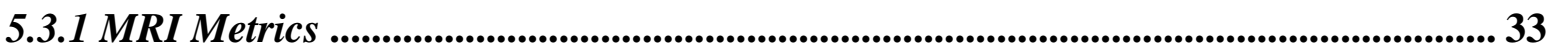

5.3.2 Cognitive Measures .......................................................................................................... 37

5.3.3 Physical Activity ................................................................................................................ 37

5.4 Exploratory Correlations with Normalized White Matter Volume .................................... 45

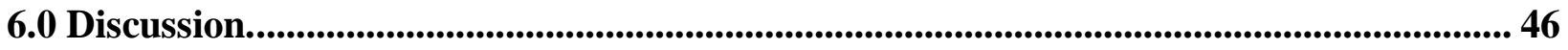

6.1 Sample Characteristics .................................................................................................................... 46

6.2 Group differences in diffusion parameters .............................................................................. 47

6.2.1 White matter integrity and lesion volume ............................................................. 49

6.3 Indices of white matter integrity not associated with cognitive measures, MRI metrics 50

6.4 Self-reported physical activity is not associated with diffusion parameters, but is

correlated to white matter volume ......................................................................................... 54

6.5 Methodological considerations ............................................................................................ 56

6.5.1 Is DTI an accurate measure of white matter integrity? ........................................... 56

6.5.2 Are diffusion parameters an accurate index of MS pathology? ................................. 57

6.5.3 Limitations of TBSS ........................................................................................................... 58

6.5.4 White matter volume

6.5.5 Complementary MRI techniques for measuring white matter ................................ 61

6.6 Limitations and Future Directions ............................................................................................ 62

6.7 Conclusions ............................................................................................................................... 62

7.0 References ............................................................................................................................64

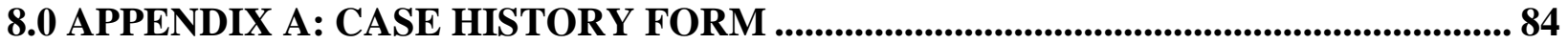

9.0 APPENDIX B. Supplementary Tables.................................................................................. 88 


\section{List of Tables}

Table 1. List of neuropsychological domains and measures .................................................... 20

Table 2. Demographic, MRI, and MS-related characteristics ........................................... 28

Table 3. Neuropsychological measures in MS patients and healthy controls ........................ 29

Table 4. Structural MRI Metrics in MS patients and healthy controls ............................... 30

Table 5. Whole Brain differences between groups in Fractional Anisotropy (FA) ${ }^{\mathrm{a}}$............. 32

Table 6. Correlations ${ }^{\mathrm{a}}$ of DTI measures and structural MRI metrics in MS patients .......... 34

Table 7. Correlations of DTI measures and lesion volume in MS patients ............................ 36

Table 8. Correlations ${ }^{\mathrm{a}}$ of DTI measures and composite cognitive scores in MS patients and

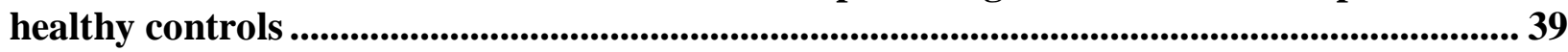

Table 9. Correlations ${ }^{\text {a }}$ of DTI measures and level of strenuous physical activity in MS patients and healthy controls .......................................................................................................... 41

Table 10. Correlations of DTI measures and composite cognitive scores in MS patients, controlling for total T1- and T2-lesion volume

Table 11. Correlations ${ }^{\mathrm{a}}$ of normalized white matter volume with strenuous physical activity, cognitive outcomes, and average DTI measures in MS patients and healthy controls

Supplementary Table 1. Correlations ${ }^{\text {a }}$ of average diffusion parameters (i.e., entire white matter skeleton) and disease characteristics in MS patients $(\mathrm{N}=23)$

Supplementary Table 2. Correlations ${ }^{\text {a }}$ of diffusion parameters and select cognitive measures in MS 


\section{List of Figures}

Figure 1. Schematic of DTI-derived diffusion parameters and how they are calculated....... 8 Figure 2. Differences in white matter FA between pediatric-onset MS patients and healthy

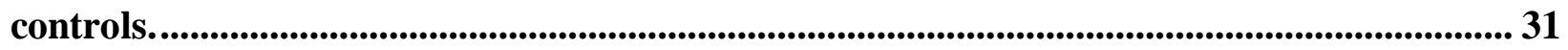

Figure 3. Hypothesis for the development of cognitive impairment in MS ........................ 52 


\section{List of Abbreviations}

9HPT: Nine Hole Peg Test

AD: Axial Diffusivity

BET: Brain Extraction Tool

BSMSS: Barratt Simplified Measure of Social Status

CES-D: Center for Epidemiological Studies Depression Scale for Children

CNS: Central Nervous System

DMT: Disease Modifying Therapy

DTI: Diffusion Tensor Imaging

EDSS: Expanded Disability Status Scale

EPI: Echo-Planar Imaging

FA: Fractional Anisotropy

fMRI: Functional Magnetic Resonance Imaging

FMRIB: Oxford Centre for Functional MRI of the Brain

FOV: Field of View

FSIQ: Full Scale IQ

FSL: FMRIB Software Library

GLTPAQ: Godin-Shepard Leisure Time Physical Activity Questionnaire

MD: Mean Diffusivity

MNI: Montreal Neurological Institute

MRI: Magnetic Resonance Imaging

MS: Multiple Sclerosis

NAWM: Normal-Appearing White Matter

NODDI: Neurite Orientation Dispersion and Density Imaging

PASAT: Paced Auditory Serial Addition Test

PD: Proton Density

PEDs-QL: Pediatric Quality of Life Inventory Multidimensional Fatigue Scale

RAVLT: Rey Auditory Verbal Learning Test

RCT: Randomized Controlled Trial

RD: Radial Diffusivity

ROI: Region of Interest 
RRMS: Relapsing Remitting Multiple Sclerosis

SES: Socioeconomic Status

SDMT: Symbol Digit Modalities Test

TBSS: Tract-Based Spatial Statistics

TFCE: Threshold-Free Cluster Enhancement

TMT-A: Trail Making Test part A

TMT-B: Trail Making Test part B

TE: Echo Time

TI: Inversion Time

TR: Repetition Time

VBM: Voxel-Based Morphometry

VO2max: Maximum Volume of Oxygen

WASI: Weschler Abbreviated Scale of Intelligence

WJ-III: Woodcock Johnson-II Battery 


\subsection{Introduction and Background}

\subsection{Overview of Multiple Sclerosis}

\subsubsection{Diagnosis and Causes}

Multiple Sclerosis (MS) is a chronic autoimmune disorder of the central nervous system (CNS) that results in neurodegeneration in the brain and spinal cord (Compston \& Coles, 2008). More specifically, MS manifests as an autoimmune attack against neurons of the CNS, and is characterized by focal and diffuse lesions (i.e., tissue damage), resulting in demyelination of these cells (Compston \& Coles, 2008). Myelin forms an insulating sheath around the axon of the neuron, and helps prevent electric current from leaving the axon (Hartline, 2008). As such, myelin is responsible for increasing the rate at which neural impulses propagate along the axon, resulting in faster and more efficient signals (Hartline, 2008). Damage to myelinated axons (i.e. white matter) results in disrupted neuronal transmission. Recent studies have noted, however, that MS is no longer characterized solely as a disease affecting white matter, but is also one that affects CNS grey matter (i.e. the cell bodies and dendrites of the neuron, from which neural impulses originate; Geurts \& Barkhof, 2008); grey matter atrophy may be due to the inflammatory response of the disease, or secondary to the effects of unstimulated axons (Chard et al., 2002).

The cause of MS is largely unknown, but may be attributed to a combination of geographic location, exposure to infection, and genetics (Marrie, 2004). Interestingly, the prevalence of MS appears to vary according to geographic latitude, with nations furthest North from the equator (i.e. North America and Northern Europe) reporting higher rates of MS than equatorial nations (i.e. Central and South America, and Asia; Koch-Henriksen \& Sørensen, 2010). Some research has postulated that level of Vitamin D (i.e., level of sun exposure) may be associated with the geographically-attributed prevalence rates, as adults with low levels of Vitamin D (e.g. individuals living further from the equator with less sun exposure) have been shown to be more susceptible to MS (Yeh et al., 2009). Other research has hypothesized genetics as a contributing factor to the impact of geographic location and MS (Milo \& Kahana, 2010). Additionally, exposure to infection, particularly Epstein-Barr Virus, has been associated with higher MS susceptibility (Yeh et al., 2009). Finally, having a family member with MS significantly increases one's risk of developing the disorder, further supporting a genetic 
association with MS (Koch-Henriksen \& Sørensen, 2010). Although identical twin studies have demonstrated genetic susceptibility in MS, with a concordance rate of approximately $25 \%$, specific genes implicated in the familial forms of the disease have not been confirmed (KochHenriksen \& Sørensen, 2010; Milo \& Kahana, 2010), although a recent study identified a genetic link to MS in a small cohort of patients (Wang et al., 2016). Taken together, it is evident that a variety of both genetic and environmental factors may influence the prevalence and development of MS.

As mentioned, MS manifests as an autoimmune attack affecting myelinated axons, resulting in lesions in the brain and spinal cord (Vishwas, Chitnis, Pienaar, Healy, \& Grant, 2010). Consequently, depending on the location of neural damage, this disorder can present in a variety of symptoms, from sensory impairment to visual and muscular impairment, as well as cognitive decline (Compston \& Coles, 2008). Unfortunately, there is no cure for this illness, warranting endeavours to establish feasible rehabilitation strategies and treatments for this patient population.

\subsubsection{Pediatric-Onset Multiple Sclerosis}

MS is typically a diagnosis given to adults, with disease onset occurring between the ages of 20 and 40 years (Chiaravalloti \& DeLuca, 2008). However, MS can be diagnosed in children and adolescents, and pediatric-onset MS (i.e., with onset under 18 years of age) represents approximately 3-4\% of all cases of MS world-wide (Yeh et al., 2009). In children, MS presents largely as a relapsing-remitting disease (such that, remission of neurological symptoms is followed by a relapse, rather than progressive neurological disability; Yeh et al., 2009), with some research demonstrating a higher relapse rate in pediatric-onset MS compared to adult MS (Banwell, Ghezzi, Bar-Or, Mikaeloff, \& Tardieu, 2007; Gorman, Healy, Polgar-Turcsanyi, \& Chitnis, 2009a). A relapse is unpredictable, and is characterized by the emergence of new symptoms and the worsening of existing ones, followed by a period of recovery (Gorman, Healy, Polgar-Turcsanyi, \& Chitnis, 2009b). Longitudinal research has noted that after a 6-year followup, children with MS continue to demonstrate elevated relapse rates in comparison to adults (Chitnis, 2013). In adults, progressive subtypes of MS also exist, with symptoms accumulating over time. In primary-progressive MS, symptoms accumulate from disease onset, whereas in the secondary-progressive subtype, symptoms accumulate 10-20 years following an initial relapsing 
disease course (Lublin et al., 2014). In children, however, progressive subtypes are very rare, with fewer than $3 \%$ of patients with pediatric-onset showing this presentation.

Along with disease progression, there are also noteworthy differences between adult and pediatric-onset MS with regards to brain morphology characteristics, as measured by magnetic resonance imaging (MRI). In contrast to adults with similar disease duration, pediatric-onset MS patients demonstrate higher lesion volume on T2 images (i.e., a type of MRI scan that is more sensitive to MS pathology, given that lesions have a higher water content than normal tissue, causing them to appear brighter on T2-weighted images; Bitar et al., 2006)), and greater tissue injury (Yeh et al., 2009). However, in spite of their higher lesion accrual, pediatric onset patients show slower disability accrual, as measured by the Expanded Disability Status Scale (EDSS; Chitnis, 2013). Moreover, the median time to reach severe disability (as indexed by a score of 4 on the EDSS), is 20 years in pediatric-onset MS, compared to 10 years in adult MS (Renoux et al., 2007).

Previous research has noted sufficient differences between pediatric-onset and adult MS. As such, further investigation of the mechanisms and symptoms that manifest in the pediatric population has been an aim of current research in this field.

\subsection{Multiple Sclerosis and the Developing Brain}

Given that the brain continues to undergo development well into young adulthood (Lebel $\&$ Beaulieu, 2011), pediatric-onset MS poses a significant risk to normal brain maturation. In contrast to adults with MS, who already have mature and established neural networks, inflammation and demyelination resulting from MS occur during the critical time when neural networks are developing in children (Portaccio et al., 2010). In typical development, a “critical period" identifies a maturational stage when the nervous system is particularly sensitive to environmental stimuli that contribute to the development of specific functions (Singleton, 2005). For example, the critical period for language acquisition in humans is believed to be before puberty (Hurford, 1991). If this period is disrupted (e.g., by a lesion to the brain), it may impact the development of these functions, resulting in impairment (such as cognitive impairment). Consequently, neurodegeneration may have a dramatic impact on cognitive functioning in pediatric-onset MS patients.

In healthy children and adolescents, increases in white matter integrity (i.e., thickening of myelin around the axons of the neurons, increases axon diameter, and better organization of 
white matter tracts) are observed with age (Barnea-Goraly, 2005). Such increases have been observed in prefrontal regions of the brain, as well as the basal ganglia, the thalamus, and projections from the corpus callosum; regions that are important for attention, motor functioning, relaying sensory information, and cognitive ability, respectively (Barnea-Goraly, 2005).

Moreover, increases in white matter integrity, particularly in frontal regions of the brain, have been observed in longitudinal studies, suggesting altered neural plasticity in different regions of the brain as one progresses through childhood and adolescence (Krogsrud et al., 2016). In adolescents, increased white matter integrity is highly correlated with cognitive and language development (Schmithorst \& Yuan, 2010), highlighting the impact of white matter integrity on cognition. Some studies have assessed neural connectivity between children and adults, and have noted greater white matter integrity in the frontal lobes of adults (aged 20-31) compared to children (aged 8-12), further reinforcing the notion that, well into adulthood, neural development continues in regions of the brain responsible for higher-order cognitive processes (Klingberg, Vaidya, Gabrieli, Moseley, \& Hedehus, 1999).

Given the continuous maturation of the brain during childhood and adolescence, there has been an increase in the application of advanced MRI techniques to better understand the impact of pediatric-onset MS on developing neural networks. MRI studies have noted declines in ageexpected brain growth among pediatric-onset MS patients (Aubert-Broche et al., 2014), along with whole brain atrophy (Kerbrat et al., 2012). Lower thalamic volumes have also been noted in pediatric-onset MS patients, compared to healthy controls (Kerbrat et al., 2012; Mesaros et al., 2008). A study conducted by Aubert-Broche et al. (2014) noted a loss of thalamic volume with disease progression (mean follow-up time: 3.55 years), as well as a failure of age-expected thalamic growth; a finding that was even more prominent in patients with higher total brain lesion load (as indexed by T2-weighted images). Voxel-based morphometry (VBM) techniques have been used to assess damage to normal appearing white matter, whereas diffusion tensor imaging (DTI) has been used to identify disruption to white matter microstructure (Blaschek et al., 2013; Tillema, Leach, \& Pirko, 2012; Vishwas et al., 2013). Through the combination of these techniques, decreased white matter integrity is consistently observed in pediatric-onset MS patients compared to healthy controls (Akbar et al., 2016; Blaschek et al., 2013). Moreover, reduced white matter integrity has been correlated with longer disease duration (Blaschek et al., 2013), and earlier age of onset (trend, Bethune et al., 2011) in pediatric-onset MS patients, 
stressing the negative impact that the illness poses on brain development, particularly for children diagnosed at a young age. Causes of these changes have been attributed to the direct negative effects that the illness has on nerve cells, considering that MS may interfere with myelination that typically continues well into adolescence, in addition to causing demyelination of mature cells (Blaschek et al., 2013). Indirect effects of the illness, such as delayed or inhibited neural maturation, have also been attributed to the observed decrease in white matter integrity (Blaschek et al., 2013).

Based on the aforementioned research, it is clear that MS diagnosed in childhood/adolescence has a detrimental impact on regional and global brain growth, with a particular impact to the development of white matter tracts. Ultimately, the harmful effects of MS on maturing neural networks have the potential to impair many brain functions, particularly cognition, as myelination of appropriate brain regions has been associated with the development of related cognitive functions (Amato et al., 2010).

\subsection{Cognitive Impairment in Pediatric-Onset MS}

\subsubsection{Progression of Cognitive Deficits}

Cognitive impairment occurs in one-third of pediatric onset MS patients, with both the prevalence and severity of impairment increasing as the disease progresses (Amato et al., 2010; Banwell \& Anderson, 2005; MacAllister et al., 2005). Cognitive impairment is one of the most debilitating aspects of the disease because of its impact on academic performance and quality of life, making prevention or remediation of cognitive dysfunction a critical goal for research in this field. Deficits are observed in a number of cognitive domains, including attention, processing speed, episodic memory, language, and problem solving (Amato et al., 2009; Banwell \& Anderson, 2005; Cardoso, Olmo, \& Fragoso, 2015; MacAllister et al., 2005).

In addition, up to $50 \%$ of patients with pediatric-onset MS report severe fatigue (as measured by the PedsQL Multidimensional Fatigue Scale; MacAllister et al., 2009). Notably, an association between both self- and parent-reported fatigue and perceived cognitive difficulties has been observed in patients (MacAllister et al., 2009).

Less longitudinal research has been conducted to examine the long-term impact that pediatric-onset MS poses on cognitive functioning. Among the longitudinal studies that have been conducted (Amato et al., 2010; Hosseini, Flora, Banwell, \& Till, 2014; MacAllister, Christodoulou, Milazzo, \& Krupp, 2007; Till et al., 2013b), loss in cognitive functioning over 
time or a failure to acquire age-expected developmental gains have been noted. For instance, declines in verbal memory, complex attention, verbal fluency, and receptive language after a 2year follow-up of pediatric-onset MS patients have been noted (Amato et al., 2010).

Additionally, greater cognitive decline over a five year period has been noted in patients with a younger age of disease onset on tests of processing speed, working memory, and visual scanning (Hosseini et al., 2014). Patients with an earlier age of onset not only demonstrated a loss of developmental gain in these cognitive domains, but also a progressive loss of cognitive function, highlighting the vulnerability of the immature brain. Similar longitudinal studies examining cognitive functioning over a one year period have noted that patients with pediatric-onset MS fail to show age-related improvements in cognition when compared to healthy controls (Till et al., 2013).

\subsubsection{Cognitive Efficiency in Pediatric-Onset MS}

Processing speed is a measure of cognitive efficiency and is defined as the ability to perform fundamental cognitive operations in an efficient manner (Kail, 2000). It is marked by pronounced age differences until adulthood and has been argued as one of the more meaningful ways to conceptualize one's mental capacity (Kail \& Salthouse, 1994). Moreover, processing speed has been postulated as a fundamental aspect of cognitive development, as it is consistently related to measures of higher-order cognition, such as fluid intelligence and short-term memory (Kail \& Salthouse, 1994). Measures of processing speed have been consistently incorporated in neuropsychological batteries evaluating cognitive functioning in pediatric-onset MS patients, as they have been shown to be sensitive to MS-related pathology (Denney, Lynch, Parmenter, \& Horne, 2004).

Age-related improvements in processing speed appear to be associated with prefrontal cortex (PFC) maturation (Rypma et al., 2006). Specifically, white matter integrity of the PFC is considerably higher in older, compared to younger, children (Asato, Terwilliger, Woo, \& Luna, 2010; Bunge, Dudukovic, Thomason, Vaidya, \& Gabrieli, 2002; Paus et al., 1999; Rypma et al., 2006). Moreover, increased frontal connectivity has been noted as a predictor of faster reaction times in children, further highlighting the importance of frontal network maturation on cognitive efficiency (Rypma et al., 2006).

Reduced information processing speed is commonly observed and manifests early in the disease as a result of diffuse damage to white matter in both pediatric-onset and adult MS 
(Banwell \& Anderson, 2005; Bergendal, Fredrikson, \& Almkvist, 2007; DeLuca, Chelune, Tulsky, Lengenfelder, \& Chiaravalloti, 2004; Denney, Lynch, Parmenter, \& Horne, 2004; Yeh et al., 2009). Bergendal et al. (2007) conducted an 8-year longitudinal study, and noted that, in adults with MS, processing speed was one of the strongest predictors of long-term cognitive decline. Additionally, DeLuca et al. (2004) noted that, compared to working memory performance, processing speed was the greatest cognitive deficit in adults with MS; it should be noted, however, that working memory performance can be exacerbated by processing speed difficulties in MS patients (Demaree, DeLuca, Gaudino, \& Diamond, 1999). In children with MS, deficits on tasks that rely upon efficient information processing have also been noted (Banwell \& Anderson, 2005). Given that white matter has a well-known role in learning and information processing (Fields, 2008), the investigation of the implications that MS has on white matter integrity in children and adolescents is warranted.

\subsection{White Matter Integrity in Pediatric-Onset MS}

\subsubsection{Measuring White Matter Integrity: Diffusion Tensor Imaging}

Measuring neural network integrity, specifically white matter integrity, is conducted by Diffusion Tensor Imaging (DTI); an MRI-based neuroimaging technique that evaluates the diffusion of water molecules within axons as an indirect measure of axonal structure (BarneaGoraly, 2005). Water diffusion in the white matter of the brain is highly sensitive to the axons' cellular membrane integrity, and as such, is able to detect differences in tissue properties that may be related to neuroinflammation (Blaschek et al., 2013). DTI is widely employed to investigate the integrity of white matter tracts, which are especially vulnerable to the pathology of MS.

There are three factors that contribute to increased white matter integrity and neural conduction. These are: dense axonal packing, large axonal diameters, and high levels of myelination (Feldman, Yeatman, Lee, Barde, \& Gaman-Bean, 2010). Through DTI, a mathematical representation of white matter integrity (as measured via water diffusion along neural axons) is produced, and is known as the "diffusion ellipsoid." The diffusion ellipsoid quantifies the magnitude and direction of hindrance to water diffusion by axons, myelin membranes, and surrounding tissue structures. Three measures of water diffusion in neural tissue are commonly reported: axial diffusivity $(\mathrm{AD})$, radial diffusivity $(\mathrm{RD})$, and fractional anisotropy (FA; Feldman et al., 2010). AD represents diffusion that is parallel to the main axis of white 
matter tracts; high $\mathrm{AD}$ is indicative of axonal loss. $\mathrm{RD}$ indicates diffusion that is perpendicular to the main axis; high RD is indicative of myelin injury. $\mathrm{AD}$ and $\mathrm{RD}$ can be combined to produce mean diffusivity (MD); a measure of the average magnitude of water diffusion. Finally, FA is the primary measure of white matter integrity and myelination. It integrates both $\mathrm{AD}$ and RD, and represents the preferential direction of diffusion. High FA values are indicative of anisotropic diffusion, as found in highly organized tissues, and low FA reflect regions of more isotropic diffusion, as would be found in cerebrospinal fluid (Feldman et al., 2010). A schematic of the DTI-derived diffusion parameters (i.e., FA, AD, RD, and MD) is outlined in Figure 1.

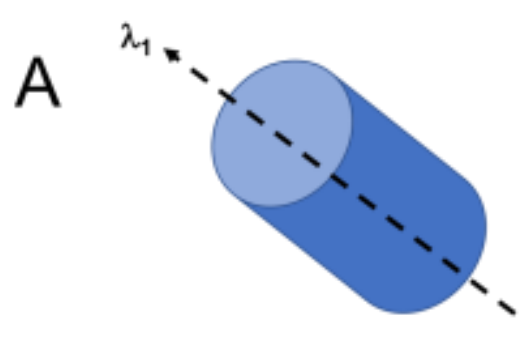

$A D=\lambda_{1}$

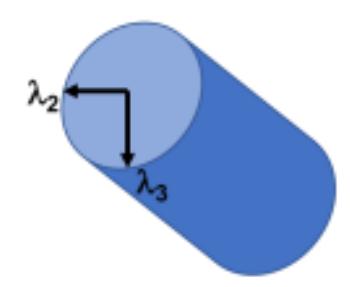

$R D=\frac{\lambda_{2}+\lambda_{3}}{2}$

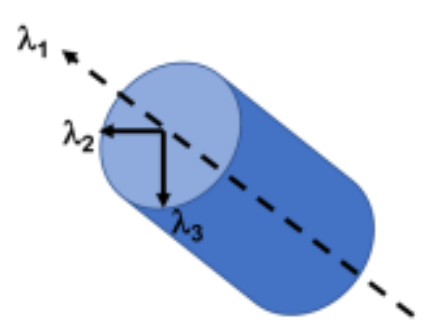

$M D=\frac{\lambda_{1}+\lambda_{2}+\lambda_{3}}{3}$

B

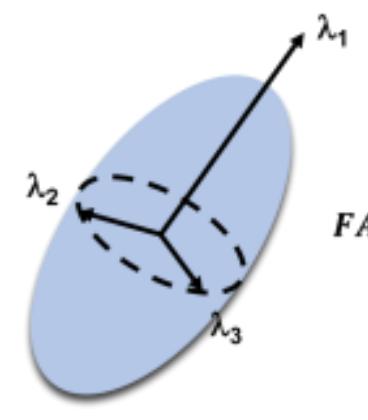

$F A=\sqrt{\frac{1}{2}} \times \frac{\sqrt{\left(\lambda_{1}-\lambda_{2}\right)^{2}+\left(\lambda_{2}-\lambda_{3}\right)^{2}+\left(\lambda_{3}-\lambda_{1}\right)^{2}}}{\left(\lambda_{1}\right)^{2}+\left(\lambda_{2}\right)^{2}+\left(\lambda_{3}\right)^{2}}$

Figure 1. Schematic of DTI-derived diffusion parameters and how they are calculated. AD represents diffusion along the length of the axon; RD is diffusion perpendicular to the length of the axon; MD is the magnitude of diffusion regardless of direction (A). The diffusion ellipsoid is shown with formula for deriving FA (i.e., indicating the principal direction of diffusion; B). Schematic derived with reference to Desouza, Hodaie, \& Davis (2014).

Measuring whole-brain differences in white matter microstructure can be facilitated by two analytic strategies: voxel-based morphometry (VBM), or tract-based spatial statistics (TBSS; Feldman et al., 2010). In voxel-based morphometry, images are registered to a standard space, and whole-brain voxelwise statistics are carried out in terms of a diffusion metric, such as FA (Feldman et al., 2010). This technique is limited by the need for spatial normalization and smoothing that may distort the brain and decrease anatomical specificity. TBSS aims to resolve 
this technical variability by aligning the FA images from multiple subjects to create a "mean FA skeleton" containing only those voxels that are assumed to be at the centre of the white matter tracts (Smith et al., 2006). Statistics are then computed at each voxel to identify regions with significant differences between groups.

\subsubsection{White Matter Changes in Pediatric-Onset MS}

Whole brain reduction in FA is consistently noted in pediatric-onset MS patients, compared to healthy controls (Akbar et al., 2016; Blaschek et al., 2013; Vishwas et al., 2010b). Regionally, loss of white matter integrity is observed in the corpus callosum (a region responsible for information-transfer between the cerebral hemispheres), suggesting that it may be an area of the brain particularly vulnerable to early-onset MS (Akbar et al., 2016; Blaschek et al., 2013; Tillema et al., 2012; Vishwas et al., 2010b). Indeed, these findings are in accordance with DTI research in adult onset MS, which have noted that the earliest changes in white matter appear in the corpus callosum (Raz et al., 2010). Rocca et al. (2014) further noted lower FA in posterior regions of the brain, such as the posterior corpus callosum and cingulum, as well as parieto-occipital white matter in cognitively-impaired pediatric MS patients. Loss of white matter integrity has been noted in other tracts, including the internal capsule (Vishwas et al., 2013), the posterior thalamic radiation, the corona radiata, and the sagittal stratum (Akbar et al., 2016). Correlations between diffusion parameters and disease-related variables have been noted in pediatric MS patients. For instance, longer disease duration has been associated with decreased FA values (Blaschek et al., 2013). Additionally, lower FA and higher AD/RD values have been associated with increased $\mathrm{T} 2$ lesion and reduced thalamic volumes (Akbar et al., 2016). Differences in normal appearing white matter are also observed between healthy controls and pediatric MS patients, with lower FA values in major white matter tracts such as the corpus callosum and superior longitudinal fasciculus (Vishwas et al., 2013). This previous research underscores the utility of measuring white matter integrity in pediatric-onset MS.

\subsubsection{White Matter Damage and Cognitive Efficiency in Pediatric-Onset MS}

\subsubsection{The role of lesions}

T1 and T2 lesion volume have been the main MRI metrics used to quantify disease severity in MS (Yeh et al., 2009). However, results show that, in general, the strength of the association between lesion volume and neuropsychological performance is weak to moderate, at best (Rocca et al., 2015; Till et al., 2012; Till et al., 2013b). This is likely attributed to the 
heterogeneity in location of lesions in white matter tracts, which is not always consistent with the clinical manifestation of the disease (Yeh et al., 2009). As previously noted, many studies have examined normally appearing white matter (NAWM) in MS, which is measured via region of interest (ROI) DTI analyses, whereby ROIs that do not contain lesions are segmented prior to extracting AD, RD, and FA values (Ciccarelli et al., 2003; Filippi et al., 2000).

\subsubsection{Damage to normal appearing white matter}

To date, only a few studies have examined the association between white matter integrity and cognitive performance in patients with pediatric-onset MS. Using DTI, microstructural white matter damage in the corpus callosum, particularly the genu and anterior body, was shown to associate strongly with reduced visual-perceptual speed (Bethune et al., 2011) and less efficient inhibition of irrelevant visual information on a global-local task (Todorow et al., 2014). Factoring out processing speed, a robust association was reported between arithmetic score and FA in white matter of the corpus callosum and in right frontal and parietal regions (Till et al., 2011b), consistent with the important role of white matter pathways underlying mathematical competence (Tsang, Dougherty, Deutsch, Wandell, \& Ben-Shachar, 2009; van Eimeren et al., 2010). Regarding white matter in the different hemispheric lobes, FA values were lower in the pediatric MS patients as compared to controls in all regions of the brain, except for the frontal lobes. The well-established caudo-rostral pattern of myelination through childhood (Paus et al., 2001; Klingberg et al., 1999) has been suggested to confer some protection of the late maturing frontal lobes from MS-related white matter insult (Bethune et al., 2011). That is, given that caudal (i.e., posterior) regions of the brain develop first, they may be more vulnerable to white matter injury. Whereas, the rostral (i.e., anterior) regions of the brain, such as the frontal cortex, have ongoing myelin maturation, allowing this region greater oppostunity to adapt to MS pathophysiology.

Evidence from other pediatric populations with white matter injury, such as traumatic brain injury (TBI), have further established the association between cognitive efficiency and white matter integrity. Previous research has noted decreased white matter integrity in pediatric TBI (Levin et al., 1997). Callosal damage has been noted at the acute stage of injury, along with evidence of long-term atrophy, and is generally related to neurocognitive outcome, including processing speed, in children with severe TBI (Verger et al., 2001; Ewing-Cobbs, Hasan, Prasad, Kramer, \& Bachevalier, 2006). In mild to moderate pediatric TBI, decreased white matter 
integrity has also been observed and has been correlated to decreased processing speed and executive functioning (Wozniak et al., 2007). Specifically, decreased frontal white matter integrity has been related to decreased executive functioning. Moreover, recent studies noted the predictive utility of DTI to identify patients with increased risk of persistent neuropsychological deficits in adult TBI (Miles et al., 2008), such that lower white matter integrity at baseline was predictive of impaired performance in planning and organization (i.e., prioritizing tasks according to a given situation) at six months post-injury (with trends for an association between slower processing speed and lower white matter integrity).

A larger body of research examining white matter integrity and cognition has been conducted in adults with MS. A study conducted by Hulst et al. (2013) was able to distinguish between cognitively impaired and cognitively preserved MS patients according to white matter integrity (as measured with DTI). They noted lower integrity of white matter as assessed by FA and $\mathrm{RD}$ in cognitively impaired patients, relative to cognitively preserved patients, in major white matter tracts (i.e., the corpus callosum, the superior and longitudinal fasciculus, the corticospinal tracts, among others). These data confirm the assumption that cognitively impaired MS patients may have more severely damaged white matter tracts.

Associations between reduced scores in tests of information processing speed and diffusion parameters have also been reported in adult MS (Dineen et al., 2009; Van Hecke et al., 2010; Yu et al., 2012). Notably, Van Hecke et al. (2010) found significant correlations between performance on the Paced Auditory Serial Addition Test (PASAT) and FA of the genu of the corpus callosum, the superior longitudinal fasciculus, the corona radiata, among other major white matter tracts. Additionally, Yu et al. (2012) noted significant correlations between performance on the Symbol Digit Modalities Test (SDMT) and widespread reduction in FA across multiple white matter tracts, with the most pronounced associations with FA of the posterior thalamic radiation, corpus callosum, and fornix.

In addition to the DTI findings, further research has noted decreased functional connectivity in resting-state networks involved in cognition, sensorimotor, and executive functions in pediatric-onset MS patients compared to healthy controls (Akbar et al., 2016; Rocca et al., 2014a). Other studies have noted neural connectivity differences between cognitivelyimpaired and cognitively-preserved pediatric-onset MS patients (Rocca et al., 2014), such that cognitively-impaired patients demonstrated decreased functional connectivity of the precuneus 
(a region involved in visuospatial and episodic memory; Rocca et al., 2014), whereas cognitively-preserved patients demonstrated increased functional connectivity of the anterior cingulate cortex (a region involved in rational cognitive functions, such as decision-making; Bush, Luu, \& Posner, 2000). Such findings provide evidence for the functional reorganization of neural networks to delay cognitive decline in pediatric-onset MS patients. Preliminary research has established a relation between white matter integrity and cognitive efficiency in both adult and pediatric-onset MS. While correlates of this relationship have been studied to a lesser degree in the pediatric population, research in adult MS has demonstrated that physical activity may be associated with white matter integrity in this population.

\subsection{Physical Activity and Brain Structure and Function}

\subsubsection{Benefits of Physical Activity in Healthy Children and Adolescents}

The beneficial effects of physical activity are well-described, and both human and animal studies have indicated that exercise is capable of improving learning capacity in both healthy and neurological populations, and assisting in restorative processes post-brain injury (Griesbach, 2011). In healthy adults, higher cardiorespiratory fitness has been associated with greater prefrontal gray matter volume (Erickson, Leckie, \& Weinstein, 2014); such effects of physical activity on the brain have also been observed in children, and have been related to higher performance on cognitive measures of working memory, inhibition, and cognitive flexibility (Chaddock, Pontifex, Hillman, \& Kramer, 2011; Chaddock et al., 2010). Moreover, ChaddockHeyman et al. (2014) noted that higher-fit 9 and 10 year-old children demonstrated greater white matter microstructure in the corpus callosum, corona radiata, and superior longitudinal fasciculus, compared to lower-fit children.

Several mechanisms through which physical activity is associated with cognitive and neurological outcomes have been studied. Brain-derived neurotrophic factor (BDNF; a protein implicated in neural survival, growth, and synaptic plasticity; Szuhany, Bugatti, \& Otto, 2015) has been postulated as one such mechanism. Neeper, Gomez-Pinilla, Choi, \& Cotman (1995) were the first to show a positive association between physical activity and BDNF mRNA levels in the rat brain, following voluntary wheel running. Increased BDNF gene expression has been noted following both voluntary and forced animal exercise protocols in the hippocampus, cerebellum, amygdala, and caudal neocortex (Voss, Vivar, Kramer, \& van Praag, 2013). 
Indeed, physical activity has been shown to elevate cerebrovascular activity and increase the release of BDNF, among other neurotrophic factors, such as nerve growth factor (NGF), growth hormone $(\mathrm{GH})$ and neurotrophin-3; proteins that stimulate neurogenesis (Archer, Svensson, \& Alricsson, 2012). In both healthy and clinical adult populations, studies have noted a relation between BDNF and cognitive processes, such as, episodic (Egan et al., 2003), and verbal memory (Grassi-Oliveira, Stein, Lopes, Teixeira, \& Bauer, 2008).

Increased vasculature (i.e., angiogenesis), and as a result, increased perfusion of blood to the brain, may be another mechanism through which physical activity confers its positive effects on cognition (Voss et al., 2013). Following a 3-month training program in adults aged 21 to 45 years, Pereira et al. (2007) noted increased cerebral blood volume to the dentate gyrus of the hippocampus. Chaddock-Heyman et al. (2016) were the first to investigate this effect in children, and noted greater blood perfusion in the hippocampus (as measured via arterial spin labelling) in higher-fit children (aged 7 to 9) versus lower-fit children.

Engagement in aerobic activity has been associated with both structural and functional brain changes, along with improved cognitive function across the lifespan. Such findings have bolstered research examining the therapeutic effects of exercise in clinical populations.

\subsubsection{Evidence from Adult MS Research}

Physical activity has been associated with greater measures of cognitive processing speed in adult MS patients (Motl, Gappmaier, Nelson \& Benedict, 2011). Briken et al. (2014) conducted a randomized controlled trial (RCT) with adult MS patients investigating three different exercise intervention techniques (arm ergometry, rowing, and bicycle ergometry). They noted improvements in verbal memory and delayed recall after 8-10 weeks of training (2-3 twenty-minute sessions/week), compared to a waitlist control group.

Accumulating research conducted in adults with MS has noted that physical activity is capable of influencing the structural and functional properties of the brain (Dalgas \& Stenager, 2012; Ibrahim et al., 2011; Prakash, Snook, Motl, \& Kramer, 2010). These studies have provided evidence for the plasticity of both gray matter regions and white matter fibers in MS, in response to physical activity. A study conducted by Prakash et al. (2010) noted that aerobic fitness was associated with increased white matter integrity in adults with MS. While this study noted that MS patients had decreased processing speed and white matter integrity than healthy controls, they observed that higher levels of fitness in patients were associated with higher scores on 
measures of processing speed, as well as greater integrity of white matter tracts in the thalamus and corpus callosum; structures that have been previously associated with cognitive impairment in MS (Prakash et al., 2010).

Another study noted changes in white matter microstructure of adult MS patients after a two-month physiotherapy intervention (Ibrahim et al., 2011). This study noted that after patients participated in two hours/week of physiotherapy for two months, increases in white matter integrity (i.e., increased FA and decreased MD from baseline to post-intervention) was observed in the corpus callosum. Furthermore, this study noted improvement in measures of working memory, processing speed, and cognitive flexibility post-intervention. Consistent with these findings, a randomized controlled trial conducted by Prosperini et al. (2014) noted increases in white matter integrity in adults with MS following a 12-week training protocol with a video game balance board (Nintendo Wii).

Together, these studies provide evidence for an association between physical activity, cognitive impairment, and white matter integrity in adult MS, and suggest possible neuroplastic mechanisms that may be responsible for the positive effects of physical activity. Such mechanisms include remyelination of white matter tracts, increases in neural connectivity, or the recruitment of new networks to complete a task (Ibrahim et al., 2011). In light of this research, exercise is becoming increasingly promoted by healthcare providers for the clinical care and management of MS (Motl \& Sandroff, 2015). However, much of the research investigating the benefits of physical activity in the MS population has been conducted on adult MS patients. A gap in the literature exists with respect to defining the relationship between physical activity, white matter integrity, and cognitive function in pediatric-onset MS patients.

\subsubsection{Preliminary Evidence in Pediatric Onset MS}

The effect of physical activity on pediatric-onset MS has yet to be fully described, though preliminary evidence suggests that exercise confers some benefits for this clinical population. Specifically, higher levels of self-reported vigorous physical activity was inversely related to fatigue in pediatric-onset MS patients, but not in controls (Yeh, Kinnett-Hopkins, Grover, \& Motl, 2015). Youth with MS are significantly less likely to be physically active compared to youth with monophasic acquired demyelinating syndrome (mono-ADS; Grover et al., 2015). Mono-ADS is a monophasic illness characterized by a single demyelinating event in the CNS, in contrast to MS, which is characterized by multiple demyelinating events (Verhey et al., 2011) 
Moreover, accelerometer data has shown that pediatric-onset MS patients participate in fewer minutes/day of vigorous physical activity, relative to youth with mono-ADS and healthy controls (Grover et al., 2015). This may be a consequence of MS being a more aggressive disease than mono-ADS. Associations have been observed between markers of disease severity and physical activity, such that increased physical activity was related to smaller T2 lesion volumes and decreased relapse rates (Grover et al., 2015); however, a causal effect of physical activity on these disease metrics cannot be inferred, as these findings were purely correlational. 


\subsection{Rationale}

Cognitive impairment is a debilitating symptom of MS that occurs in roughly one-third of children and adolescents with MS. Currently, there is little known about modifiable factors that may contribute to the preservation of neural networks and underlying cognitive processes in pediatric-onset MS. Research in adult MS has noted that physical activity may be associated with decreased disease pathology, though the extent of this effect has not been studied in pediatriconset MS. Exploring the potential benefits of physical activity in individuals with pediatriconset MS is a worthwhile endeavor, as these patients may be especially vulnerable to the disruption of white matter pathways and cognitive decline. Moreover, investigating the neural mechanisms underlying the benefits of exercise for pediatric-onset MS is important for understanding potential protective factors of the disease process. Research is therefore needed to identify whether physical activity is associated with white matter integrity and cognition in the pediatric-onset MS population. 


\subsection{Objectives and Hypotheses}

Objective: The proposed study aimed to measure the relation between white matter integrity, cognitive efficiency, and self-reported, strenuous physical activity in patients with pediatric-onset MS. A supplementary aim compared the strength of this relationship across patients and healthy controls.

Hypothesis A: Whole brain white matter integrity was expected to be lower in pediatriconset MS patients compared to healthy controls. Lower white matter integrity was also expected to be lower in pediatric-onset MS patients in tracts that have been identified as more vulnerable to MS pathology (i.e., the corpus callosum and projections from the thalamus).

Hypothesis B: Cognitive efficiency was expected to be lower in pediatric-onset MS patients, compared to healthy controls

Hypothesis $C$ : Higher reports of strenuous physical activity were expected to be associated with higher whole brain white matter integrity and greater cognitive efficiency. This association was also expected to be observed in major white matter tracts, including the corpus callosum the superior longitudinal fasciculus, and the posterior thalamic radiation (which have been observed as particularly vulnerable to MS disease pathology). 


\subsection{Method}

This study was approved by the Research Ethics Board at York University and the Hospital for Sick Children. Participants older than 16 years provided informed consent, whereas younger participants provided verbal assent; parents of younger participants provided written informed consent.

\subsection{Participants}

This study examined previously-collected data from a sample of 25 pediatric-onset relapsing remitting MS patients (aged 14-25). Patients were recruited for this study from the Pediatric Demyelinating clinic at the Hospital for Sick Children (Toronto, ON). Patients were informed of the study by letter, followed by a telephone screen by a research assistant. Thirtyeight age- and gender-matched healthy controls were recruited from the Undergraduate Research Participant Pool (URPP) at York University, and from local advertisements (i.e., flyers).

All participants recruited for this study were between 13-25 years of age. All patients were required to meet the revised McDonald 2010 MS diagnostic criteria (Polman et al., 2011) for relapsing-remitting MS, and to be younger than 18 years of age at the time of the first MS attack. MS patients were also required to be at least four weeks from clinical relapse or corticosteroid treatment. These criteria were implemented, as visual/motor disturbances and MRI abnormalities have been noted during cognitive testing and MRI during clinical relapse. Moreover, declines in processing speed have been observed in patients experiencing a relapse (Benedict et al., 2014).

The following exclusion criteria were implemented: 1) history of significant head trauma (i.e., loss of consciousness for more than 5 minutes and a score of less than 13 on the Glasgow Coma Scale); 2) history of alcohol abuse, or illicit drug use (greater than once per week); 3) major medical illness that could affect cognitive functioning; 4) visual or motor difficulties that would preclude testing; 5) lack of proficiency in English. History of a mood-related disorder (e.g., depression) was not exclusionary, as such symptoms may be secondary to the MS disease process. Apart from MS diagnosis, age- and sex-matched healthy controls met the same inclusion/exclusion criteria; however, controls were not recruited if they reported history of neurological illness, psychiatric diagnosis, or known learning disability. To limit practice effects, all participants were required to be more than 6 months from any previous neuropsychological evaluation. 


\subsection{Study Procedure}

Upon receiving a study information letter, and agreeing to be contacted by the research assistant, participants were screened (by telephone, or in clinic) via a screening form to ensure that inclusion and exclusion criteria were met. Participants also completed an MRI screening form prior to testing, to ensure that they were eligible to enter the scanner.

Testing occurred at York University and lasted approximately 3 hours, with breaks. The testing procedure included questionnaires to collect information regarding demographics, mood, and fatigue. Participants then completed a 60-minute neuropsychological test battery. Finally, an MRI scan was conducted using a Siemens MAGNETOM 3T Tim Trio MRI scanner at York University. Scans lasted approximately 90 minutes for patients, and 70 minutes for controls. All participants were reimbursed $\$ 20$ for parking/public transportation.

\subsection{Measures}

\subsubsection{Clinical-Demographic Information}

Clinical information was obtained for patients through chart review or communication with their neurologist, on the following outcomes within 3 months of the study: age at disease onset (i.e., age at first MS attack), total number of relapses, medication use, and physical disability (assessed by a neurologist via the Expanded Disability Status Scale [EDSS]; Kurtzke, 1983).

The following information was collected from all participants via questionnaires: 1) general health and demographic information (Case History Form; Appendix A); 2) handedness (Dutch Handedness Questionnaire; Strien, 1992); 3) mood (Center for Epidemiologic Studies Depression Scale [CES-D]; Faulstich, Carey, Ruggiero, Enyart, \& Gresham, 1986); 4) three dimensions of fatigue - General, Cognitive, and Sleep/Rest (Varni Pediatric Quality of Life [PedsQL] Multidimensional Fatigue Scale; Varni, Seid, \& Rode, 1999); 5) socioeconomic status, as determined by parental education and occupational status (Barratt Simplified Measure of Social Status; Barratt, 2005); and 6) physical activity (Godin-Shephard Leisure-Time Exercise Questionnaire; Godin, 2011).

\subsubsection{Neuropsychological Battery}

A battery of clinical neuropsychological tests was employed to assess the following cognitive domains: general intellectual function (IQ), focused attention and cognitive flexibility, 
cognitive efficiency/speed of processing, learning and memory, and motor function (patients only). A list of the neuropsychological measures is presented in Table $\mathbf{1 .}$

Table 1. List of neuropsychological domains and measures

DOMAIN

INTELLECTUAL FUNCTION

FOCUSED ATTENTION AND

COGNITIVE FLEXIBILITY

SPEED OF PROCESSING

LEARNING \& MEMORY

MOTOR FUNCTION (PATIENTS)
MEASURE

Wechsler Abbreviated Scales of Intelligence (WASI) two subtest estimate of Full Scale IQ (Wechsler, 1999)

- Vocabulary: general word knowledge

- Matrix Reasoning: nonverbal conceptual reasoning

Trail Making Test - Parts A and B (Reitan, 1959)

Woodcock Johnson (WJ-III): Auditory Working Memory (Schrank, 2010)

Symbol Digits Modalities Test (SDMT) - Oral Version (A. Smith, 1968)

WJ-III: Decision speed (Woodcock, McGrew, \& Mather, 2001)

Rey Auditory Verbal Learning Test (RAVLT; Lezak, 1983)

Nine Hole Peg Test (9-HPT) (norms from Mathiowetz, Weber, Kashman, \& Volland, 1985)

\subsubsection{Speed of Processing Measures}

This study focused on the neuropsychological measures that evaluated speed of processing. These included the Symbol Digit Modalities Test (SDMT) (oral version), and the Woodcock Johnson (WJ-III) Decision Speed subtest. The SDMT requires participants to orally match symbols to numbers according to a key within 90 seconds. It measures attention, concentration, and speed of processing (Smith, 1991). This task has demonstrated strong testretest reliability in a cohort of 85 adult MS patients who were tested monthly for 5 sessions (Benedict et al., 2008). It is known to be sensitive in distinguishing MS patients from healthy controls, and for detecting cognitive impairment in adult MS patients (Lopez-Gongora et al., 2015; Parmenter et al., 2007; Schependom et al., 2014). Moreover, scores on the SDMT have 
been shown to correlate with other measures of neuropsychological performance, such as memory and executive function (Lopez-Gongora et al., 2015). In pediatric-onset MS, the SDMT score has been sensitive to noting decline in processing speed over time (Till et al., 2013), and has been associated with age of disease onset (Till et al., 2012). Performance on this test is also associated with indices of disease burden, including global and regional brain MRI metrics, and to a lesser extent, T1- and T2-lesion volumes (Till et al., 2011). Because of its sensitivity to detecting cognitive impairment, the SDMT is one of the core tests in the validated Brief Neuropsychological Battery for Children (BNBC) (Portaccio et al., 2009).

To complement the SDMT, a secondary measure of processing speed, the WJ-III Decision Speed subtest, was assessed. This task assesses semantic processing speed and requires participants to quickly locate two pictures that are most similar conceptually, within a 3-minute time limit (Schrank, 2010). It has demonstrated good test-retest reliability (above 0.70) across age groups (Schrank \& Woodcock, 2001). Although it has not been used extensively in pediatriconset MS, it provides complementary information regarding processing speed within the cohort involved in this study.

A cognitive efficiency composite score was generated for each participant by calculating the mean of the SDMT and the WJ-III Decision Speed scores. A neuropsychological composite score was estimated for each participant by calculating the mean z-score of seven subtests from the test battery (i.e., RAVLT total score, TMT-B, WASI Vocabulary and Matrix Reasoning, SDMT, WJ-III Decision Speed and Auditory Working Memory).

\subsubsection{Assessment of Physical Activity Level}

The Godin-Shephard Leisure-Time Physical Activity Questionnaire (GLTPAQ) was used to assess physical activity level. This questionnaire is a self-report measure that asks subjects to recall the amount of times in the last 7 days that they spent more than 15 minutes doing exercise that is classified as mild (i.e., requiring minimal effort), moderate (i.e., not exhausting), or strenuous (i.e., causing the heart to beat rapidly). For each classification, examples of exercises are provided. From these weekly frequencies, a "weekly leisurely activity", or leisure index score can be derived. The questionnaire also queries the number of times per week that one engages in physical activity long enough to work up a sweat.

The GLTPAQ has shown good reliability and validity in previous studies (Jacobs, Ainsworth, Hartman, \& Leon, 1993; Sallis, Buono, Roby, Micale, \& Nelson, 1993). High test- 
retest reliability has been noted for the leisure index score $(r=0.74$, Godin \& Shephard, 1985; 0.81, Sallis et al., 1993) over a 2-week testing period. Godin \& Shephard (1985) further noted high test-retest reliability for the measure of self-reported strenuous exercise (0.94), with lower reliability for self-reported mild and moderate exercise ( $r=0.48$ and 0.46 , respectively). A similar study was conducted with adults aged 20-59 (Jacobs et al., 1993). Over a one-month interval, they noted high test-retest reliability of the leisure index $(r=0.62)$ and self-reported strenuous exercise $(r=0.84)$. Lower test-retest reliability was noted for self-reported mild or moderate exercise ( $r=0.24$ and 0.36 , respectively). This study also found that the leisure index was positively associated with motion score as assessed using an accelerometer (metabolic equivalent in min/day), and maximum oxygen consumption ( $\left.\mathrm{VO}_{2} \mathrm{max}\right)$. Moreover, the leisure index was negatively correlated with total body fat. Godin \& Shephard (1985) also observed that the measure of strenuous exercise was moderately correlated to $\mathrm{VO}_{2}$ max $(r=0.38)$.

As mentioned previously, the GLTPAQ has been used to evaluate the association between physical activity and disease activity in pediatric-onset MS and monophasic demyelinating syndrome (mono-ADS) patients (Grover et al., 2015). MS patients reported participating in less strenuous physical activity compared to mono-ADS patients; groups did not differ in moderate or mild PA. Another study by the same group observed correlations between accelerometry data and the GLTPAQ measure in children with demyelination (Grover et al., 2015).

Based on this previous work, this study examined self-reported strenuous exercise and as the measure of interest for estimating physical activity among participants.

\subsubsection{MRI Protocol}

All data were acquired on a Siemens MAGNETOM 3T Tim Trio MRI scanner at York University with a 32-channel head coil. The 90-minute scanning protocol included the following: T1-weighted sagittal MPRAGE, resting state functional MRI (fMRI), three task-based fMRI paradigms, DTI, proton-density (PD), T2-weighted, and FLAIR sequences. The T1-weighted three-dimensional MPRAGE sequence [1 mm isotropic voxel size, TR=2300 ms, TE=2.96 ms, field of view $(\mathrm{FOV})=256 \times 240 \times 192 \mathrm{~mm}$, number of slides $=192]$ was used to measure brain volume, register images, and perform anatomical mapping. DTI images were recorded using a 2D echo-planar imaging (EPI) sequence with diffusion weighting in 64 directions and b-value of $1000 \mathrm{~s} / \mathrm{mm}^{2}(\mathrm{TR}=4600 \mathrm{~ms}, \mathrm{TE}=93 \mathrm{~ms}, \mathrm{FOV}=256 \times 256 \times 108 \mathrm{~mm}$, number of slices=36, voxel 
size $=2.0 \times 2.0 \times 3.0 \mathrm{~mm})$. The $\mathrm{PD},(\mathrm{TR}=2200 \mathrm{~ms}, \mathrm{TE}=10 \mathrm{~ms}$, turbo factor=4) and T2-weighted $(\mathrm{TR}=4500 \mathrm{~ms}, \mathrm{TE}=83 \mathrm{~ms}$, turbo factor=11) images were acquired for lesion segmentation using 2D turbo spin-echo sequences with $1 \times 1 \times 3 \mathrm{~mm}^{3}$ voxel size, along with a matching $2 \mathrm{D}$ turbo FLAIR sequence with $\mathrm{TR}=9000 \mathrm{~ms}, \mathrm{TE}=88 \mathrm{~ms}, \mathrm{TI}=2407.5 \mathrm{~ms}$. The resting state and task-based fMRI data were collected as part of other studies conducted in the same population (Akbar et al. 2015; 2016).

\subsubsection{Lesion Segmentation}

Image processing and structural MRI analyses were performed at the Montreal Neurological Institute (MNI; Montreal, QC) by trained staff who were blinded to clinical and behavioural data.

A preprocessing routine was run on all images to correct for intensity non-uniformity (Sled, Zijdenbos, \& Evans, 1998), to remove skull and scalp (Smith, 2002), and to linearly register the T1-weighted images to the PD- and T2-weighted image to provide voxel-wise anatomical alignment across the modalities (Collins, Neelin, Peters, \& Evans, 1994). Second, the intensity range was normalized within each image, using a two-piece linear transformation as described in Nyul and Udupa (1999). Third, using an interactive, mouse-driven visualization software package (DISPLAY, McConnell Brain Imaging Centre, Montreal Neurological Institute), the T2-weighted lesion labels output by an initial automated segmentation procedure were superimposed on the T1-, T2-, and PD-weighted images, carefully reviewed and, if necessary, manually corrected. Given their different MRI intensity characteristics, infratentorial T2-weighted lesions were segmented manually. Hypointense regions on T1-weighted images (T1-weighted lesions) located within the T2-weighted lesions were automatically segmented by applying an intensity threshold of $85 \%$ relative to the mean intensity of surrounding normalappearing WM. Supratentorial and infratentorial lesion volumes were combined as a measure of total brain lesion volume.

\subsubsection{Brain and Thalamus Segmentation}

Three standard preprocessing steps were applied to the native T1-weighted images to delineate the thalamus. These included: (1) removing noise by using the optimized non-local means filter (Coupe, Hellier, Prima, Kervrann, \& Barillot, 2008); (2) reducing the impact of intensity inhomogeneity due to RF coil variations using a non-parametric estimation of the slow varying non-uniformity field (Sled et al., 1998), and (3) normalizing the brain volume intensities 
to the intensities of the target template [i.e., the ICBM152 population template (Fonov et al., 2011)] by doing regression based analyses on image intensity percentiles.

The following steps were then conducted on the preprocessed images: (1) a mixture of linear and non-linear transformation (Collins et al., 1994) of the raw T1-weighted image to ICBM152 population template space (Fonov et al., 2011), (2) brain extraction using a library of tissue priors that are available in the ICBM152 population template (Eskildsen et al., 2012), (3) warping of the thalamus onto the raw T1-weighted image using the inverse of the transformation generated in step (1). Thalamic volumes were normalized for head size by dividing by total intracranial volume.

Normalized whole-brain GM and WM volumes were estimated using SIENAX (Smith 2001, Smith 2002b). First, the brain and skull were extracted from the T1 image (Smith 2002). The brain image was then affine-registered to Montreal Neurological Institute (MNI)152 space, using the skull image to determine the registration scaling (Jenkinson 2001, Jenkinson 2002). Subsequently, tissue-type segmentation with partial volume estimation was carried out (Zhang 2001 ) in order to calculate total volume of brain tissue.

\subsection{Analyses}

\subsubsection{DTI Processing}

DTI processing was conducted at York University using FMRIB Software Library (FSL; www.fmrib.ox.ac.uk/fs1/). DTI images were examined for quality control in fslview prior to preprocessing, and slices which demonstrated noise artifacts were removed (one slice was removed in two participant scans).

The preprocessing protocol was as follows; first, DTI data were corrected for MRI eddy currents and head motion using affine registration to a reference volume (i.e., where $b=0$ ). Brain masks were generated using the Brain Extraction Tool (BET) and entered into the DTIFIT program, which creates a diffusion tensor model at each voxel. As a result of preprocessing, images representing fractional anisotropy (FA), axial diffusivity (AD), and radial diffusivity (RD) were created for each subject on a voxel-wise level.

In order to conduct voxel-wise between-group comparisons of the DTI data, Tract-Based Spatial Statistics (TBSS) was employed (Smith et al., 2006). As mentioned previously, in TBSS, the whole brain is investigated, and prespecification of tracts of interest is not required. This 
method estimates a "group mean FA skeleton" representing the centres of all of the fibre bundles of the subjects included in the analysis.

Individual FA images were aligned to a $1 \times 1 \times 1 \mathrm{~mm}^{3}$ standard space, via nonlinear registration. This was followed by the creation of a mean FA image that was used to create the mean FA white matter skeleton (set at a threshold of 0.2 ). The white matter skeleton includes only the voxels that are assumed to be at the centre of the fiber bundles, and excludes regions that are poorly aligned (Feldman et al., 2010). The FA values for each subject were projected onto the mean FA skeleton. Subsequently, FA differences between patients and healthy controls were compared at a voxel-wise level, controlling for age and sex. Group differences were determined via permutation-based testing in FSL Randomise. Threshold-free cluster enhancement (TFCE) was applied, as it provides a voxel-wise significance that is corrected for multiple comparisons (Blaschek et al., 2013). This procedure was also conducted for AD and RD values.

To examine the probability that a voxel was a member of a specific tract of interest, FSL's atlasquery function was employed. White matter structures were defined by FSL's JHU white matter tractography atlas (Mori \& Zhang, 2006). Only structures which were identified by atlasquery as having a minimum probability of 0.5 or greater were included in further analyses. FA values were subsequently extracted from the identified white matter tracts via FSL's fslmaths and fslmeants commands.

\subsubsection{Statistical Analyses}

The Shapiro-Wilk and Levene's tests were used to detect violations of normality and homogeneity of variance within the data set. Descriptive statistics were performed for the clinical characteristics of our MS sample (i.e., age at disease onset, disease duration, number of relapses, EDSS score, use of disease-modifying therapies, T1 and T2 total lesion volumes). Groups were compared on demographic variables (age, sex, years education, socioeconomic status), as well as on depressive symptom classification, fatigue score, cognitive efficiency composite score, selfreported strenuous physical activity level, and diffusion parameters using independent samples $t$ tests, Mann-Whitney $U$ tests, or Chi-square tests, where appropriate (alpha of 0.05). The cognitive outcomes of interest included the cognitive efficiency and neuropsychological composite scores; exploratory analyses included measures of fine motor dexterity (i.e., dominant and non-dominant hand performance on the 9-hole pegboard task). Outliers (i.e., scores that lie 
above or below three standard deviations from the normative mean) were trimmed using a Winsorizing procedure to three standard deviations above/below the mean. Cognitive impairment was defined as performance 1.5 standard deviations below average on three or more subtests out of the seven measures that were used to derive the neuropsychological composite score. Self-reported strenuous physical activity for each participant was derived from the GLTPAQ (i.e., the number of times/week that they participated in over 15 minutes of strenuous exercise where their heart beats rapidly). The outcome measures of interest for the DTI data included FA, AD, and RD values for the entire white matter skeleton, as well as FA values for tracts identified via atlasquery in FSL.

Pearson partial correlations (two-tailed, adjusted for age; alpha of 0.01) were conducted to examine the association between the outcome measures of interest. Supplementary analyses examining the relation between diffusion parameters and other MRI measures (i.e., brain and thalamic volume, and lesion volume) were also conducted. Exploratory Pearson partial correlations (two-tailed, adjusted for age, T1- and T2 lesion volume; alpha of 0.01) were conducted to further investigate the association between the cognitive composite scores and diffusion parameters. 


\subsection{Results}

\subsection{Sample Characteristics}

In total, 38 healthy controls and $25 \mathrm{MS}$ patients were recruited and participated in the study. Two MS patients (8\%) and two controls (5.3\%) in the study sample met criteria for cognitive impairment. Four MS patients (16\%) and two controls (5.3\%) met criteria for major depression. In order to ensure that the control sample was as representative of a healthy sample as possible, the participants who met criteria for cognitive impairment and major depression were excluded from any further analyses $(\mathrm{N}=4)$, leaving a total of 34 controls. Of note, only 24 of 34 controls completed the GLPTAQ (because the questionnaire was introduced late into the study). MS patients were not excluded from the analyses based on cognitive impairment or selfreported major depression given that these factors are associated with MS disease pathology (Yeh et al., 2009). Upon inspection of T1-weighted MRI scans, two MS patients demonstrated large topographical changes in brain structures due to extremely large ventricles or a massive lesion. These scans were excluded from the DTI analyses, as previous research has demonstrated that pronounced morphological changes in the brain affect the projection step in TBSS and will impact the quality of the subject's alignment to the white matter skeleton (Bach et al., 2014). Thus, the overall sample size for the MS group consisted of 23 MS patients.

Demographic and disease-related characteristics of the sample are reported in Table 2. Groups did not differ significantly on measured demographic variables, including age at scan, sex, years of education, and parental socioeconomic status (SES). Groups also did not differ on measures of fatigue or depression symptom classification. Contrary to the hypothesis, MS patients and controls did not differ in self-reported strenuous physical activity. 
Table 2. Demographic and MS-related characteristics

\begin{tabular}{|c|c|c|c|c|}
\hline & $\begin{array}{l}\text { MS } \\
(n=23)\end{array}$ & $\begin{array}{l}\text { Control } \\
(n=34)\end{array}$ & $\begin{array}{l}\text { Cohen's } \\
\text { d }\end{array}$ & p-value \\
\hline Mean (SD) Age at scan, yrs & $18.45(2.76)$ & $18.55(2.98)$ & 0.03 & 0.89 \\
\hline $\operatorname{Sex}(\mathbf{F} / \mathbf{M})$ & $17 / 6$ & $24 / 10$ & & $0.78^{\mathrm{a}}$ \\
\hline Mean (SD) Years education & $12.48(2.45)$ & $12.71(2.46)$ & 0.09 & 0.73 \\
\hline Mean (SD) SES ${ }^{c}$ & $44.63(13.93)$ & $45.54(15.04)$ & 0.06 & $0.68^{\mathrm{b}}$ \\
\hline Mean (SD) Disease Duration ${ }^{d}$, months & $53.39(32.30)$ & & & \\
\hline Mean (SD) Age at MS Onset, years & $13.43(2.76)$ & & & \\
\hline Mean (SD) Number of Relapses & $4.13(3.18)$ & & & \\
\hline $\begin{array}{l}\text { Median (range) Physical Disability } \\
\text { rating, EDSS }\end{array}$ & $1.5(1.0-6.0)$ & & & \\
\hline DMT use $(\mathbf{Y} / \mathbf{N})$ & $18 / 5$ & & & \\
\hline $\begin{array}{l}\text { Depression Symptom Classification }{ }^{\mathrm{e}} \text { (n: } \\
\text { Normal/Mild/Major) - CES-DC }\end{array}$ & $15 / 4 / 4$ & $25 / 9 / 0$ & & $0.29^{\mathrm{a}}$ \\
\hline Mean (SD) Fatigue F $^{\mathrm{f}}$ & $59.52(18.89)$ & $65.81(11.16)$ & 0.51 & 0.16 \\
\hline $\begin{array}{l}\text { Mean (SD) Level of Strenuous Physical } \\
\text { Activityg }\end{array}$ & $2.66(1.86)$ & $2.96(2.03)$ & 0.15 & $0.76^{\mathrm{b}}$ \\
\hline \multicolumn{5}{|c|}{ 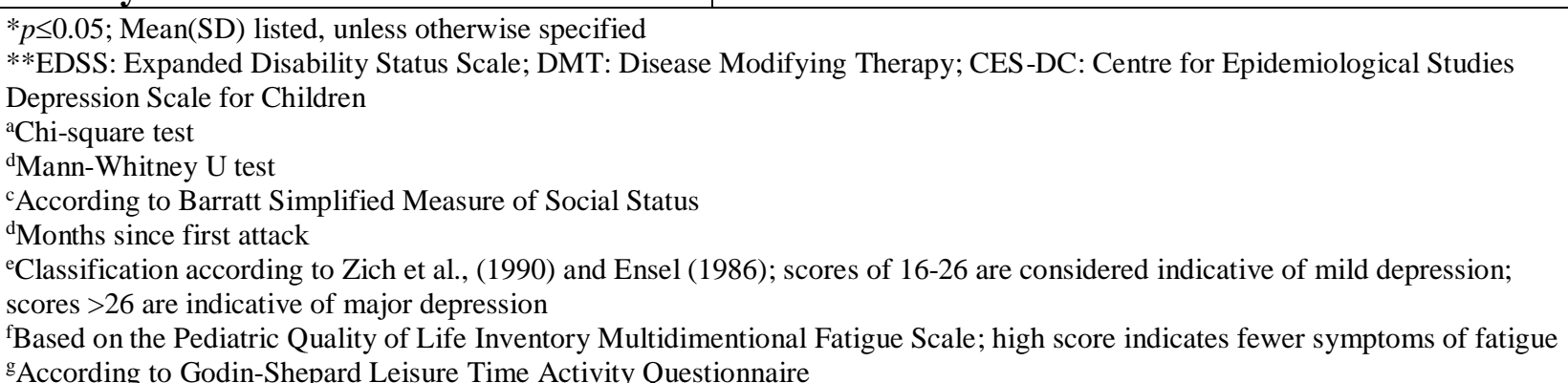 } \\
\hline
\end{tabular}


Performance on the battery of neuropsychological tests is reported in Table 3. MS patients exhibited a significantly lower Full Scale IQ (FSIQ; measured via the WASI) compared to healthy controls $(p=0.039)$; however FSIQ was within normal limits for both groups. Patients also demonstrated poorer performance on the WASI Vocabulary subtest ( $p=0.024)$, as well as poorer fine motor coordination on the 9HPT ( $p=0.018$ ), relative to controls, and only with the dominant hand. Groups did not differ significantly on any other cognitive tasks, nor on the neuropsychological composite or cognitive efficiency composite scores.

Table 3. Neuropsychological measures in MS patients and healthy controls

\begin{tabular}{l|llll} 
& \multicolumn{1}{|c}{$\begin{array}{c}\text { MS } \\
(\mathbf{n = 2 3})\end{array}$} & $\begin{array}{c}\text { Control } \\
(\mathbf{n = 3 4})\end{array}$ & Cohen's d & $\boldsymbol{p}$ value \\
\hline Neuropsychological Composite & $0.04(0.75)$ & $0.24(0.55)$ & 0.36 & 0.26 \\
Cognitive Efficiency Composite & $0.30(0.93)$ & $0.25(1.10)$ & 0.05 & 0.88 \\
SDMT & $0.20(1.00)$ & $0.43(1.42)$ & 0.19 & 0.47 \\
WJ-III Decision Speed & $-0.08(0.83)$ & $0.035(1.05)$ & 0.12 & 0.66 \\
RAVLT - Total Immediate Recall & $-0.20(1.09)$ & $-0.36(1.21)$ & 0.14 & 0.62 \\
RAVLT - Delayed Recall & $-0.19(1.00)$ & $0.051(1.06)$ & 0.23 & $0.28^{\mathrm{a}}$ \\
WASI Matrix Reasoning & $0.09(0.87)$ & $0.37(0.56)$ & 0.38 & $0.27^{\mathrm{a}}$ \\
WASI Vocabulary & $0.23(0.88)$ & $0.72(0.70)$ & 0.62 & $\mathbf{0 . 0 2 4}$ \\
WASI Full Scale IQ & $0.17(0.77)$ & $0.58(0.63)$ & 0.58 & $\mathbf{0 . 0 3 9}$ \\
WJ-III Auditory Working Memory & $0.26(0.64)$ & $0.32(0.67)$ & 0.09 & 0.74 \\
TMT-A & $0.16(1.16)$ & $0.39(0.88)$ & 0.22 & $0.43^{\mathrm{a}}$ \\
TMT-B & $-0.40(1.74)$ & $0.13(0.95)$ & 0.38 & $0.60^{\mathrm{a}}$ \\
Nine Hole Peg Test (Dominant & $-1.17(1.26)$ & $-0.44(0.77)$ & 0.70 & $\mathbf{0 . 0 1 8 ^ { \mathrm { a } }}$ \\
Hand) & & & & \\
Nine Hole Peg Test (Non-Dominant & $-1.10(1.22)$ & $-0.79(0.85)$ & 0.29 & 0.32 \\
Hand) & & & & \\
\hline
\end{tabular}

${ }^{*} p \leq 0.05 ;$ Mean(SD) reported

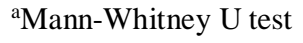

Note: SDMT: Symbol Digits Modalities Test; WJ-III: Woodcok Johnson III Test of Cognitive Abilities $-3^{\text {rd }}$ Edition; RAVLT:

Rey Auditory Verbal Learning Test; WASI: Weschler Abbreviated Scales of Intelligence; TMT-A/B: Trail Making Tests Parts A and $\mathrm{B}$ 
A summary of the MRI characteristics are noted in Table 4. MS patients demonstrated smaller normalized white matter volume $(p=0.009)$, and normalized thalamic volume $(p<0.001)$ than controls. Normalized grey matter volume and total brain volume did not differ between groups. Lesion volume in the MS group was highly variable with T2 volume ranging from 0.006 to $25.13 \mathrm{~cm}^{3}$ (median 4.10 ) and $\mathrm{T} 1$ lesion volume ranging from 0 to $12.1 \mathrm{~cm}^{3}$ (median 2.03 ).

Table 4. Structural MRI Metrics in MS patients and healthy controls

\begin{tabular}{|c|c|c|c|c|}
\hline & $\begin{array}{c}\text { MS } \\
(n=23)\end{array}$ & $\begin{array}{c}\text { Control } \\
(n=34)\end{array}$ & $\begin{array}{c}\text { Cohen's } \\
\text { d }\end{array}$ & $\begin{array}{c}p \\
\text { value }\end{array}$ \\
\hline Brain Volume (z-score) & $-0.61(0.84)$ & $-0.60(1.14)$ & 0.010 & 0.98 \\
\hline $\begin{array}{l}\text { Normalized Grey Matter Volume } \\
\left(\mathrm{cm}^{3}\right)\end{array}$ & $851.40(35.74)$ & $872.70(45.86)$ & 0.052 & 0.066 \\
\hline $\begin{array}{l}\text { Normalized White Matter Volume } \\
\left(\mathrm{cm}^{3}\right)\end{array}$ & $691.83(34.73)$ & $721.16(40.64)$ & 0.78 & $0.009^{a}$ \\
\hline $\begin{array}{l}\text { Normalized Thalamic Volume z- } \\
\text { score (median \& range) }\end{array}$ & $0.03(-3.40-1.83)$ & $1.28(-0.53-2.69)$ & 1.36 & $<.001$ \\
\hline $\begin{array}{l}\text { Total T2 Lesion Volume }\left(\mathrm{cm}^{3} \text {; }\right. \\
\text { median \& range) }\end{array}$ & $4.10(0.006-25.13)$ & -- & & -- \\
\hline $\begin{array}{l}\text { Total } \mathrm{T} 1 \text { Lesion Volume }\left(\mathrm{cm}^{3} \text {; }\right. \\
\text { median \& range) }\end{array}$ & $2.03(0.00-12.1)$ & -- & & -- \\
\hline
\end{tabular}

$* p \leq 0.05 ;$ Mean(SD) listed, unless otherwise specified

aMann-Whitney U test

\subsection{Diffusion Tensor Imaging (DTI) Results}

Results of the TBSS analysis revealed that MS patients demonstrated lower FA compared to the control group within the entire white matter skeleton $(p<0.001$; Figure 2). MS patients exhibited lower FA (all p values < 0.006) in several specific white matter tracts, including the genu, body, and splenium of the corpus callosum, the bilateral cerebral peduncles, the left retrolenticular part of the internal capsule, the left anterior corona radiata, the bilateral superior corona radiata, the bilateral posterior corona radiata, the bilateral posterior thalamic radiation, the bilateral sagittal stratum, the bilateral external capsule, and the bilateral fornix (Table 5). MS patients also demonstrated greater $\mathrm{AD}$ compared to the control group in the entire white matter skeleton $(p<.001)$, as well as greater RD $(p<.001)$. 


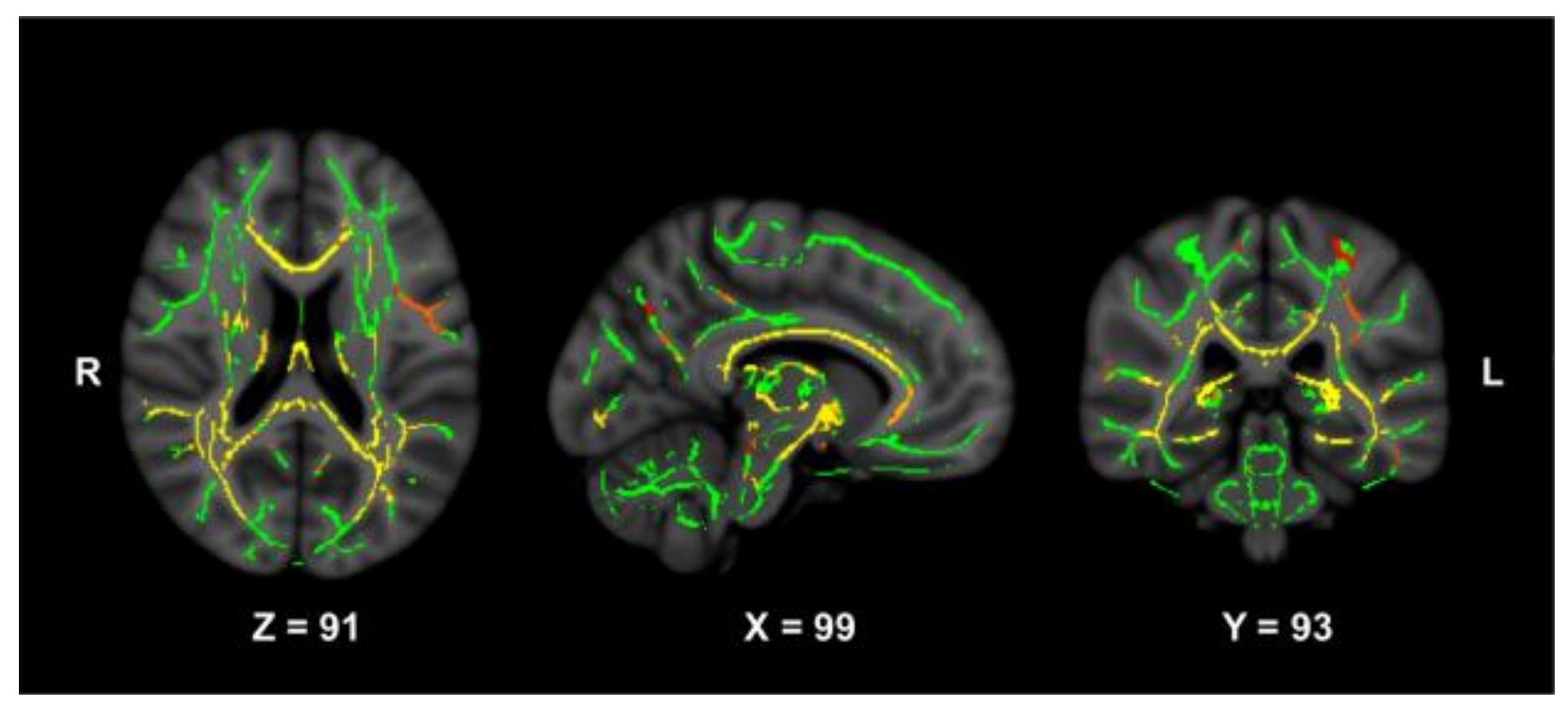

Figure 2. Differences in white matter FA between pediatric-onset MS patients and healthy controls. White matter skeleton depicted in green. Areas in which patients demonstrated lower FA compared to controls are shown in red and yellow ( $p<.05$, TFCE corrected). 
Table 5. Whole Brain differences between groups in diffusion parameters ${ }^{\mathrm{a}}$

\begin{tabular}{|c|c|c|c|c|}
\hline & $\begin{array}{c}\text { MS } \\
(n=23)\end{array}$ & $\begin{array}{c}\text { Control } \\
(n=34)\end{array}$ & $\begin{array}{c}\text { Cohen's } \\
\text { d }\end{array}$ & $\begin{array}{c}p \\
\text { value }\end{array}$ \\
\hline \multicolumn{5}{|l|}{ Fractional Anisotropy } \\
\hline Entire white matter skeleton & $0.510(0.023)$ & $0.552(0.017)$ & 2.08 & $<.001$ \\
\hline Corpus Callosum - Genu & $0.642(0.042)$ & $0.678(0.031)$ & 1.46 & $<.0011^{\mathrm{b}}$ \\
\hline Corpus Callosum - Body & $0.598(0.047)$ & $0.661(0.032)$ & 1.57 & $<.001$ \\
\hline Corpus Callosum - Splenium & $0.729(0.026)$ & $0.764(0.018)$ & 1.57 & $<.001$ \\
\hline Cerebral Peduncle - Right & $0.666(0.031)$ & $0.707(0.026)$ & 1.43 & $<.001^{\mathrm{b}}$ \\
\hline Cerebral Peduncle - Left & $0.708(0.030)$ & $0.748(0.029)$ & 1.36 & $<.001^{\mathrm{b}}$ \\
\hline Internal Capsule - Right Anterior Limb & $0.566(0.026)$ & $0.581(0.028)$ & 0.56 & .030 \\
\hline Internal Capsule - Right Posterior Limb & $0.649(0.025)$ & $0.663(0.029)$ & 0.52 & .030 \\
\hline Internal Capsule - Left Posterior Limb & $0.652(0.024)$ & $0.657(0.028)$ & 0.19 & .22 \\
\hline Internal Capsule - Right Retrolenticular Part & $0.546(0.033)$ & $0.564(0.026)$ & 0.61 & .011 \\
\hline Internal Capsule - Left Retrolenticular Part & $0.561(0.030)$ & $0.582(0.029)$ & 0.71 & .006 \\
\hline Corona Radiata - Right Anterior & $0.442(0.039)$ & $0.459(0.031)$ & 0.48 & .042 \\
\hline Corona Radiata - Left Anterior & $0.420(0.029)$ & $0.444(0.032)$ & 0.79 & .003 \\
\hline Corona Radiata - Right Superior & $0.465(0.026)$ & $0.484(0.019)$ & 0.83 & .002 \\
\hline Corona Radiata - Left Superior & $0.463(0.022)$ & $0.481(0.018)$ & 0.90 & $<.001$ \\
\hline Corona Radiata - Right Posterior & $0.447(0.028)$ & $0.484(0.027)$ & 1.35 & $<.001$ \\
\hline Corona Radiata - Left Posterior & $0.433(0.028)$ & $0.463(0.022)$ & 1.19 & $<.001$ \\
\hline Posterior Thalamic Radiation - Right & $0.557(0.046)$ & $0.622(0.024)$ & 1.77 & $<.001^{\mathrm{b}}$ \\
\hline Posterior Thalamic Radiation - Left & $0.555(0.040)$ & $0.621(0.026)$ & 1.96 & $<.001^{\mathrm{b}}$ \\
\hline Saggital Stratum - Right & $0.503(0.038)$ & $0.550(0.026)$ & 1.44 & $<.001$ \\
\hline Saggital Stratum - Left & $0.514(0.031)$ & $0.553(0.025)$ & 1.38 & $<.001$ \\
\hline External Capsule - Right & $0.449(0.021)$ & $0.466(0.024)$ & 0.75 & .004 \\
\hline External Capsule - Left & $0.434(0.019)$ & $0.451(0.020)$ & 0.87 & .001 \\
\hline Fornix - Right & $0.496(0.032)$ & $0.554(0.041)$ & 1.58 & $<.001$ \\
\hline Fornix - Left & $0.501(0.046)$ & $0.559(0.027)$ & 1.54 & $<.001$ \\
\hline Superior Longitudinal Fasciculus - Right & $0.464(0.026)$ & $0.478(0.020)$ & 0.60 & .025 \\
\hline Superior Longitudinal Fasciculus - Left & $0.464(0.032)$ & $0.482(0.023)$ & 0.65 & $.017^{\mathrm{b}}$ \\
\hline \multicolumn{5}{|l|}{ Axial Diffusivity $\left(1^{-3} \mathbf{m m}^{2}\right)$} \\
\hline Entire White Matter Skeleton & $1.32(0.049)$ & $1.25(0.038)$ & 1.60 & $<.001$ \\
\hline \multicolumn{5}{|l|}{ Radial Diffusivity $\left(10^{-3} \mathrm{~mm}^{2}\right)$} \\
\hline Entire White Matter Skeleton & $0.604(0.036)$ & $0.544(0.026)$ & 1.91 & $<.001^{\mathrm{b}}$ \\
\hline
\end{tabular}

$* p \leq 0.01$; one-tailed: lower FA in MS compared to healthy controls; higher AD and RD in MS compared to controls

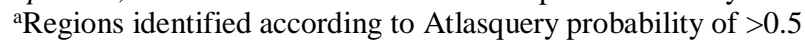

${ }^{\mathrm{b}}$ Mann-Whitney U test 


\subsection{DTI Correlation Analyses}

Diffusion parameters for the entire white matter skeleton (i.e.., average FA, AD, and RD) were not correlated (two-tailed Pearson partial correlations, adjusted for age; alpha of 0.01) with any of the following clinical characteristics: disease duration, age of disease onset, number of relapses, EDSS score, and fatigue (Supplementary Table 1; Appendix B). FA of specific white matter tracts also did not correlate with the above measures.

\subsubsection{MRI Metrics}

The relation between MRI metrics and DTI measures was examined in MS patients (twotailed Pearson partial correlations, adjusted for age; alpha of 0.01; Table 6). Brain volume did not show any associations with FA, AD, or RD values. Normalized thalamic volume was positively associated with FA of the right superior longitudinal fasciculus $(r=0.705, p<.001)$. Normalized white matter volume in the brain was negatively associated with FA of the right posterior limb of the internal capsule $(r=-0.712, p<.001)$. Normalized grey matter volume was negatively associated with FA of the right superior corona radiata $(r=-0.563, p=0.008)$ and with FA of the left posterior corona radiata $(r=-0.560, p=.008)$.

Further associations between lesion volume and DTI measures were examined in MS patients (Table 7). Both T1 and T2 lesion volume showed negative correlations with the entire white matter skeleton FA (T2: $r=-0.613, p=0.003$; T1: $r=-0.655, p=0.001)$. Significant negative correlations with T1 lesion volumes were also noted with FA of the body and splenium of the corpus callosum. The FA of the left superior corona radiata, the bilateral posterior corona radiata, and the left sagittal stratum were associated with both T2 and T1 lesion volume; T1 lesion volume was additionally associated with FA of the right sagittal stratum (Table 7). T1 and T2 lesion volume were positively associated with RD (T2: $r=0.602, p=0.004 ; \mathrm{T} 1: r=0.625$, $p=0.002)$; no associations between lesion volume and AD were noted. 
Table 6. Correlations ${ }^{\mathrm{a}}$ between DTI measures and structural MRI metrics in MS patients

\begin{tabular}{|c|c|c|c|c|}
\hline & $\begin{array}{l}\text { Normalized } \\
\text { Grey Matter } \\
\text { Volume }\left(\mathbf{c m}^{3}\right)\end{array}$ & $\begin{array}{l}\text { Normalized } \\
\text { White Matter } \\
\text { Volume }\left(\mathbf{c m}^{3}\right)\end{array}$ & $\begin{array}{l}\text { Normalized } \\
\text { Thalamic } \\
\text { Volume } \\
\text { (z-score) }\end{array}$ & $\begin{array}{l}\text { Brain Volume } \\
\text { (z-score) }\end{array}$ \\
\hline \multicolumn{5}{|l|}{ Fractional Anisotropy } \\
\hline Entire white matter skeleton & -0.249 & -0.067 & 0.200 & -0.249 \\
\hline Corpus Callosum - Genu ${ }^{\mathrm{b}}$ & 0.014 & 0.276 & -0.097 & 0.179 \\
\hline Corpus Callosum - Body & -0.065 & 0.026 & 0.100 & -0.256 \\
\hline Corpus Callosum - Splenium & $-0.464 *$ & -0.294 & 0.109 & -0.344 \\
\hline Cerebral Peduncle - Right ${ }^{\mathrm{b}}$ & -0.163 & -0.283 & 0.148 & -0.023 \\
\hline Cerebral Peduncle - Left ${ }^{\mathrm{b}}$ & 0.038 & -0.036 & -0.108 & 0.149 \\
\hline Internal Capsule - Right Anterior Limb & -0.050 & -0.063 & -0.064 & -0.152 \\
\hline Internal Capsule - Right Posterior Limb & $-0.484 *$ & $-0.712 * * *$ & $0.395^{*}$ & $-0.520^{*}$ \\
\hline Internal Capsule - Left Posterior Limb & -0.254 & -0.340 & 0.102 & $-0.430 *$ \\
\hline Internal Capsule - Right Retrolenticular Part & -0.070 & -0.134 & $0.397 *$ & $-0.450^{*}$ \\
\hline Internal Capsule - Left Retrolenticular Part & -0.095 & -0.035 & 0.309 & -0.203 \\
\hline Corona Radiata - Right Anterior & -0.047 & 0.200 & -0.227 & 0.045 \\
\hline Corona Radiata - Left Anterior & -0.118 & 0.048 & 0.083 & -0.189 \\
\hline Corona Radiata - Right Superior & $-0.563 * *$ & $-0.432 *$ & 0.262 & $-0.453^{*}$ \\
\hline Corona Radiata - Left Superior & $-0.545^{*}$ & $-0.442 *$ & 0.306 & $-0.518^{*}$ \\
\hline Corona Radiata - Right Posterior & $-0.397 *$ & -0.023 & 0.271 & -0.331 \\
\hline Corona Radiata - Left Posterior & $-0.560 * *$ & -0.163 & 0.020 & -0.273 \\
\hline Posterior Thalamic Radiation - Right ${ }^{\mathrm{b}}$ & -0.267 & -0.238 & $0.394 *$ & -0.236 \\
\hline Posterior Thalamic Radiation - Left ${ }^{\mathrm{b}}$ & -0.290 & -0.040 & -0.126 & -0.037 \\
\hline Saggital Stratum - Right & -0.248 & -0.007 & 0.136 & -0.073 \\
\hline Saggital Stratum - Left & -0.274 & -0.183 & 0.217 & -0.286 \\
\hline External Capsule - Right & -0.278 & -0.033 & 0.088 & -0.286 \\
\hline External Capsule - Left & -0.133 & 0.177 & -0.027 & -0.217 \\
\hline Fornix - Right & 0.015 & 0.153 & 0.334 & -0.103 \\
\hline
\end{tabular}




\begin{tabular}{|c|c|c|c|c|}
\hline Fornix - Left & -0.122 & 0.234 & 0.148 & 0.195 \\
\hline Superior Longitudinal Fasciculus - Right & -0.257 & -0.178 & $0.705 * * *$ & -0.228 \\
\hline Superior Longitudinal Fasciculus - Left ${ }^{\mathrm{b}}$ & -0.114 & 0.333 & $0.525^{*}$ & -0.334 \\
\hline Axial Diffusivity & & & & \\
\hline Entire White Matter Skeleton & -0.041 & 0.196 & -0.210 & $0.403^{*}$ \\
\hline Radial Diffusivity & & & & \\
\hline Entire White Matter Skeleton ${ }^{\mathrm{b}}$ & 0.147 & 0.098 & -0.239 & $0.396 *$ \\
\hline
\end{tabular}

${ }^{\mathrm{a}} \mathrm{r}$-value reported

$* p \leq 0.1$ (trend); $* * p<0.01 ; * * * p<0.001$

bLog-transformed 
Table 7. Correlations between DTI measures and lesion volume in MS patients

\begin{tabular}{|c|c|c|}
\hline & $\begin{array}{l}\text { Total T2 Lesion } \\
\text { Volume }\left(\mathrm{cm}^{3}\right)^{b} \\
\end{array}$ & $\begin{array}{l}\text { Total T1 Lesion } \\
\text { Volume }\left(\mathrm{cm}^{3}\right)^{\mathbf{b}}\end{array}$ \\
\hline \multicolumn{3}{|l|}{ Fractional Anisotropy } \\
\hline Entire white matter skeleton & $-0.613 * *$ & $-0.655 * *$ \\
\hline Corpus Callosum - Genu ${ }^{\mathrm{b}}$ & -0.364 & $-0.400 *$ \\
\hline Corpus Callosum - Body & $0.541 *$ & $-0.585 * *$ \\
\hline Corpus Callosum - Splenium & $-0.532 *$ & $-0.562 * *$ \\
\hline Cerebral Peduncle - Right ${ }^{b}$ & -0.292 & -0.329 \\
\hline Cerebral Peduncle - Left & 0.179 & 0.140 \\
\hline Internal Capsule - Right Anterior Limb & 0.132 & 0.069 \\
\hline Internal Capsule - Right Posterior Limb & -0.055 & -0.009 \\
\hline Internal Capsule - Left Posterior Limb & -0.135 & -0.096 \\
\hline Internal Capsule - Right Retrolenticular Part & -0.297 & -0.311 \\
\hline Internal Capsule - Left Retrolenticular Part & -0.094 & -0.150 \\
\hline Corona Radiata - Right Anterior & -0.203 & -0.238 \\
\hline Corona Radiata - Left Anterior & $-0.418 *$ & $-0.474 *$ \\
\hline Corona Radiata - Right Superior & $-0.507 *$ & $-0.500 *$ \\
\hline Corona Radiata - Left Superior & $-0.596^{*}$ & $-0.571 *$ \\
\hline Corona Radiata - Right Posterior & $-0.707 * * *$ & $-0.720 * * *$ \\
\hline Corona Radiata - Left Posterior & $-0.548 * *$ & $-0.542 * *$ \\
\hline Posterior Thalamic Radiation - Right ${ }^{\mathrm{b}}$ & $-0.417 *$ & $0.423 *$ \\
\hline Posterior Thalamic Radiation - Left ${ }^{b}$ & $-0.497 *$ & $0.505^{*}$ \\
\hline Saggital Stratum - Right & $-0.547 *$ & $-0.583 * *$ \\
\hline Saggital Stratum - Left & $-0.642 * *$ & $-0.667 * *$ \\
\hline External Capsule - Right & 0.080 & 0.092 \\
\hline External Capsule - Left & 0.190 & 0.155 \\
\hline Fornix - Right & -0.327 & -0.362 \\
\hline Fornix - Left & -0.300 & $-0.412 *$ \\
\hline Superior Longitudinal Fasciculus - Right & -0.292 & -0.281 \\
\hline Superior Longitudinal Fasciculus - Left ${ }^{\mathrm{b}}$ & -0.299 & -0.306 \\
\hline \multicolumn{3}{|l|}{ Axial Diffusivity } \\
\hline Entire White Matter Skeleton & $0.469 *$ & $0.459 *$ \\
\hline \multicolumn{3}{|l|}{ Radial Diffusivity } \\
\hline Entire White Matter Skeleton ${ }^{b}$ & $0.602 * *$ & $0.625 * *$ \\
\hline
\end{tabular}

${ }^{a}$ Log-transformed; r-value reported

${ }^{*} p \leq 0.1$ (trend); $* * p<0.01 ; * * * p<0.001$ 


\subsubsection{Cognitive Measures}

Correlations between composite cognitive scores and diffusion parameters were examined in MS patients and controls. The cognitive efficiency and the neuropsychological composite scores did not significantly relate to any diffusion parameters, in either MS patients or controls (Table 8). However, several trends were observed. In the MS group, and contrary to expectation, the neuropsychological composite score tended to negatively correlate with FA of the bilateral external capsule (Right: $r=-0.490, p=0.024$; Left: $r=-0.496, p=0.022$ ), and the right superior longitudinal fasciculus ( $r=-0.377, p=0.092$ ). These negative associations became slightly stronger when controlling for $\mathrm{T} 1$ and $\mathrm{T} 2$ lesion volume, though significance levels remained at trend level (i.e., $p>0.01$; Table 10). In contrast to the neuropsychological composite score, the cognitive efficiency composite tended to positively associate with FA of the body of the corpus callosum ( $r=0.456, p=0.038)$, and negatively associate with the right superior longitudinal fasciculus $(r=-0.380, p=0.090)$. In the control group, only one trend was noted for a positive association between the cognitive efficiency composite and FA of the right external capsule $(r=0.316, p=0.073)$.

As an exploratory analysis, correlations between FA and individual measures of cognitive efficiency (i.e., SDMT and Decision Speed) were conducted in MS patients. No associations were revealed. The association between FA and psychomotor speed (Nine-Hole Pegboard Test; dominant hand) was also examined given that this measure was significantly lower in the MS group relative to controls, and that fine motor dexterity has been previously associated with FA of the corpus callosum and association fibres (i.e., cingulum, inferior longitudinal fasciculus, and uncinated fasciculus; Zahr, Rohlfing, Pfefferbaum, \& Sullivan, 2008). No significant association was found (Supplementary Table 2, Appendix B).

\subsubsection{Physical Activity}

When Pearson partial correlations of the DTI data with self-reported strenuous physical activity were conducted, several trends (in the expected direction) were observed (Table 9). Specifically, in the MS group, physical activity tended to positively associate with FA of the right anterior limb of the internal capsule ( $r=.401, p=.072$ ), and negatively associate with FA of the right posterior limb of the internal capsule $(r=-.496, p=.022)$. In the control group, physical activity tended to positively associate with FA of the genu $(r=.494, p=.017)$, body ( $r=.396$, $p=.062)$, and splenium $(r=.400, p=.059)$ of the corpus callosum, and the left anterior corona 
radiata ( $r=.379, p=.074)$. Additionally, $\mathrm{RD}$ of the entire white matter skeleton tended to negatively associate with physical activity in controls $(r=-.389, p=.066)$. When controlling for T1- and T2-lesion volume in MS patients, positive trends were noted with FA of the genu of the corpus callosum $(r=0.440, p=0.060)$ and the right anterior corona radiata $(r=0.434, p=0.063)$, and a negative trend with FA of the right posterior limb of the internal capsule $(r=-0.398$, $p=0.091 ;$ Table 10). 
Table 8. Correlations ${ }^{\mathrm{a}}$ between DTI measures and composite cognitive scores in MS patients and healthy controls

\begin{tabular}{|c|c|c|c|c|}
\hline & \multicolumn{2}{|c|}{ Neuropsychological Composite } & \multicolumn{2}{|c|}{ Cognitive Efficiency Composite } \\
\hline & MS $(n=23)$ & Control $(n=34)$ & MS & Control \\
\hline \multicolumn{5}{|l|}{ Fractional Anisotropy } \\
\hline Entire white matter skeleton & 0.205 & -0.051 & 0.323 & 0.118 \\
\hline Corpus Callosum - Genu ${ }^{\mathrm{b}}$ & 0.267 & 0.024 & 0.283 & 0.075 \\
\hline Corpus Callosum - Body & 0.317 & -0.110 & $0.456 *$ & 0.074 \\
\hline Corpus Callosum - Splenium & 0.229 & -0.075 & 0.286 & 0.095 \\
\hline Cerebral Peduncle - Right ${ }^{\mathrm{b}}$ & 0.012 & 0.276 & 0.051 & 0.258 \\
\hline Cerebral Peduncle - Left $^{\mathrm{b}}$ & -0.244 & 0.138 & -0.174 & 0.304 \\
\hline Internal Capsule - Right Anterior Limb & 0.153 & -0.018 & 0.380 & 0.067 \\
\hline Internal Capsule - Right Posterior Limb & -0.174 & -0.079 & -0.193 & 0.128 \\
\hline Internal Capsule - Left Posterior Limb & -0.264 & -0.165 & -0.241 & 0.098 \\
\hline Internal Capsule - Right Retrolenticular Part & -0.041 & 0.058 & 0.026 & 0.148 \\
\hline Internal Capsule - Left Retrolenticular Part & -0.225 & -0.219 & -0.274 & -0.026 \\
\hline Corona Radiata - Right Anterior & 0.067 & 0.037 & 0.216 & 0.133 \\
\hline Corona Radiata - Left Anterior & 0.212 & -0.019 & 0.362 & -0.025 \\
\hline Corona Radiata - Right Superior & -0.028 & -0.145 & 0.068 & 0.074 \\
\hline Corona Radiata - Left Superior & 0.042 & -0.141 & 0.115 & 0.025 \\
\hline Corona Radiata - Right Posterior & 0.126 & -0.068 & 0.270 & 0.206 \\
\hline Corona Radiata - Left Posterior & 0.240 & -0.120 & 0.335 & 0.022 \\
\hline Posterior Thalamic Radiation - Right $^{\mathrm{b}}$ & 0.225 & 0.055 & 0.323 & 0.113 \\
\hline Posterior Thalamic Radiation $-\mathrm{Left}^{\mathrm{b}}$ & 0.195 & -0.080 & 0.242 & -0.013 \\
\hline Saggital Stratum - Right & -0.020 & 0.290 & 0.006 & 0.280 \\
\hline Saggital Stratum - Left & 0.065 & -0.160 & 0.190 & 0.062 \\
\hline External Capsule - Right & $-0.490 *$ & 0.252 & -0.301 & $0.316^{*}$ \\
\hline External Capsule - Left & $-0.496^{*}$ & -0.051 & -0.230 & 0.108 \\
\hline Fornix - Right & -0.256 & 0.206 & -0.084 & 0.322 \\
\hline Fornix - Left & -0.009 & -0.073 & -0.033 & 0.118 \\
\hline Superior Longitudinal Fasciculus - Right & $-0.377 *$ & -0.147 & $-0.380 *$ & -0.050 \\
\hline
\end{tabular}




\begin{tabular}{l|llll} 
Superior Longitudinal Fasciculus - Left ${ }^{\mathrm{b}}$ & -0.144 & -0.063 & -0.068 & -0.046 \\
\hline $\begin{array}{l}\text { Axial Diffusivity } \\
\text { Entire White Matter Skeleton }\end{array}$ & -0.210 & -0.078 & -0.307 & -0.101 \\
\hline $\begin{array}{l}\text { Radial Diffusivity } \\
\text { Entire White Matter Skeleton }{ }^{\mathrm{b}}\end{array}$ & -0.197 & 0.034 & -0.321 & -0.097 \\
\hline${ }^{\mathrm{a}}$ r-value reported
\end{tabular}

${ }^{\mathrm{a}} \mathrm{r}$-value reported

${ }^{*} p \leq 0.1$ (trend); $* * p<0.01 ; * * * p<0.001$

${ }^{b}$ Log-transformed 
Table 9. Correlations ${ }^{\text {a }}$ between DTI measures and level of strenuous physical activity in MS patients and healthy controls

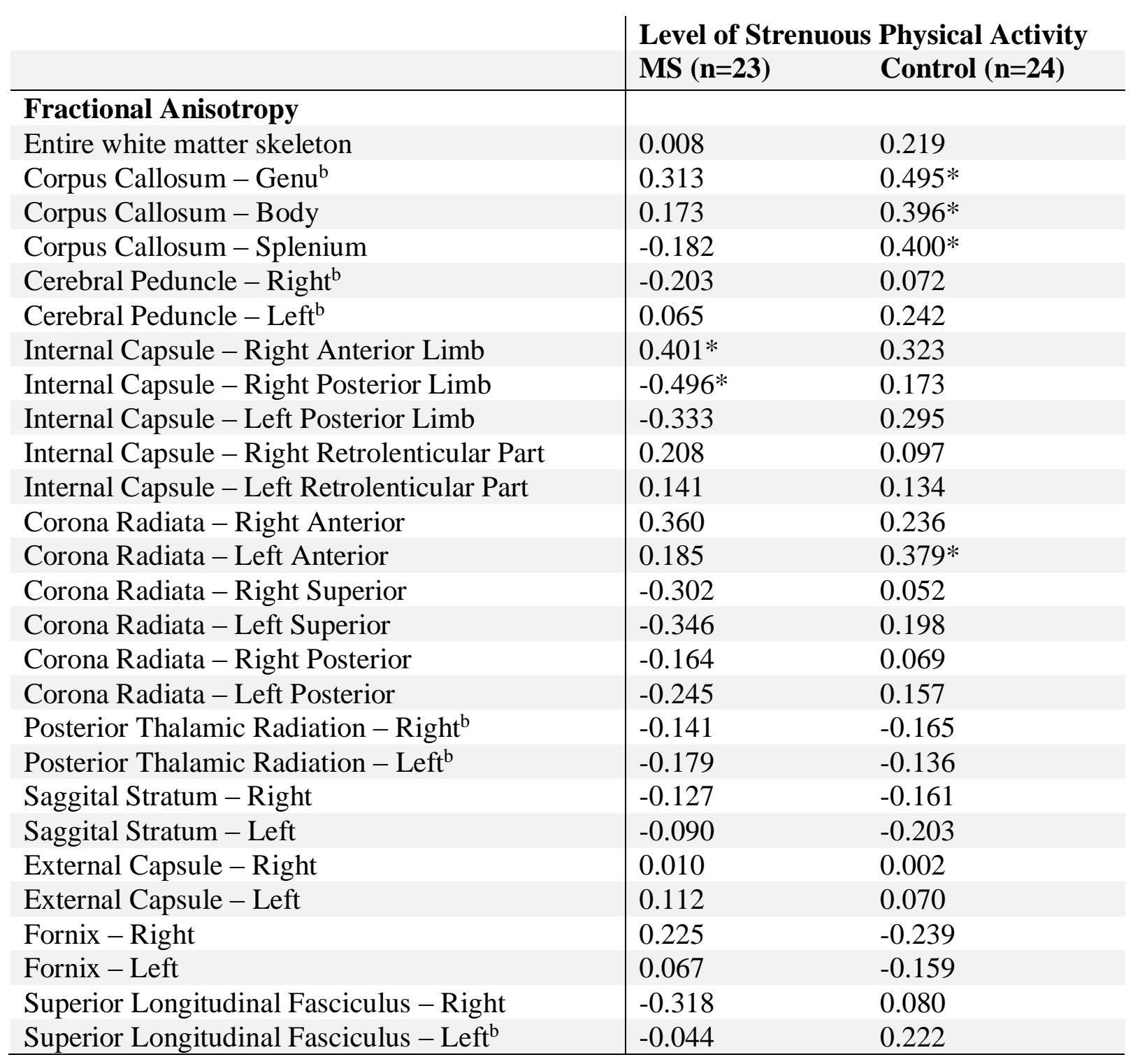




\begin{tabular}{l|ll}
\hline Axial Diffusivity & & \\
Entire White Matter Skeleton & 0.099 & -0.267 \\
\hline Radial Diffusivity & & \\
Entire White Matter Skeleton ${ }^{\mathrm{b}}$ & 0.003 & $-0.389^{*}$ \\
\hline${ }^{\mathrm{a}}$-value reported
\end{tabular}

${ }^{a} \mathrm{r}$-value reported

${ }^{*} p \leq 0.1$ (only trends shown)

${ }^{b}$ Log Transformed 
Table 10. Correlations between DTI measures and composite cognitive scores in MS patients, controlling for total T1- and T2-lesion volume

\begin{tabular}{|c|c|c|c|}
\hline & $\begin{array}{l}\text { Neuropsychological } \\
\text { Composite }\end{array}$ & $\begin{array}{l}\text { Cognitive Efficiency } \\
\text { Composite }\end{array}$ & $\begin{array}{l}\text { Self-Reported } \\
\text { Strenuous Physical } \\
\text { Activity }\end{array}$ \\
\hline \multicolumn{4}{|l|}{ Fractional Anisotropy } \\
\hline Entire white matter skeleton & -0.049 & -0.049 & 0.067 \\
\hline Corpus Callosum - Genu ${ }^{\mathrm{b}}$ & 0.104 & 0.007 & $0.440 *$ \\
\hline Corpus Callosum - Body & 0.115 & 0.173 & 0.210 \\
\hline Corpus Callosum - Splenium & 0.093 & 0.099 & -0.151 \\
\hline Cerebral Peduncle - Right ${ }^{\mathrm{b}}$ & -0.028 & 0.015 & -0.152 \\
\hline Cerebral Peduncle - Left ${ }^{\mathrm{b}}$ & -0.183 & -0.073 & 0.046 \\
\hline Internal Capsule - Right Anterior Limb & 0.090 & 0.355 & 0.330 \\
\hline Internal Capsule - Right Posterior Limb & -0.070 & 0.023 & $-0.398 *$ \\
\hline Internal Capsule - Left Posterior Limb & -0.285 & -0.297 & -0.296 \\
\hline Internal Capsule - Right Retrolenticular Part & -0.129 & -0.081 & 0.367 \\
\hline Internal Capsule - Left Retrolenticular Part & -0.210 & -0.290 & 0.221 \\
\hline Corona Radiata - Right Anterior & -0.242 & -0.271 & $0.434 *$ \\
\hline Corona Radiata - Left Anterior & -0.074 & -0.078 & 0.199 \\
\hline Corona Radiata - Right Superior & -0.231 & -0.182 & -0.226 \\
\hline Corona Radiata - Left Superior & -0.153 & -0.143 & -0.311 \\
\hline Corona Radiata - Right Posterior & -0.055 & -0.082 & -0.081 \\
\hline Corona Radiata - Left Posterior & 0.069 & 0.105 & -0.257 \\
\hline Posterior Thalamic Radiation - Right ${ }^{\mathrm{b}}$ & 0.055 & 0.098 & -0.108 \\
\hline Posterior Thalamic Radiation - Left ${ }^{\mathrm{b}}$ & 0.013 & -0.041 & -0.155 \\
\hline Saggital Stratum - Right & -0.087 & -0.059 & 0.028 \\
\hline Saggital Stratum - Left & -0.198 & -0.156 & 0.012 \\
\hline External Capsule - Right & $-0.521 *$ & -0.360 & 0.062 \\
\hline External Capsule - Left & $-0.560 *$ & -0.337 & 0.018 \\
\hline Fornix - Right & -0.332 & -0.183 & 0.314 \\
\hline Fornix - Left & -0.049 & -0.114 & 0.101 \\
\hline Superior Longitudinal Fasciculus - Right & $-0.392 *$ & -0.319 & -0.085 \\
\hline
\end{tabular}


Superior Longitudinal Fasciculus - Left ${ }^{\mathrm{b}}$ $-0.171$ $-0.096$ 0.015

Axial Diffusivity

Entire White Matter Skeleton

0.040

0.118

0.246

Radial Diffusivity

Entire White Matter Skeleton ${ }^{\mathrm{b}}$

0.095

0.136

0.015

${ }^{*} p \leq 0.1$ (only trends shown)

bog-transformed 


\subsection{Exploratory Correlations with Normalized White Matter Volume}

Exploratory Pearson partial correlations (two-tailed, adjusted for age; alpha of 0.01) revealed that normalized white matter volume was associated with self-reported strenuous physical activity in controls $(r=0.531, p=0.009)$; a trend for a positive association was noted in MS patients ( $r=0.442, p=0.045$; Table 11). No associations were found with normalized white matter and the neuropsychological composite, or cognitive efficiency. Exploratory analyses examining the relation between normalized white matter and FA, $\mathrm{AD}$, and $\mathrm{RD}$ values of the entire white matter skeleton also did not reveal any significant associations.

Table 11. Correlations ${ }^{a}$ between normalized white matter volume and strenuous physical activity, cognitive outcomes, and average DTI measures in MS patients and healthy controls

\begin{tabular}{l|ll} 
& \multicolumn{2}{|l}{ Normalized White Matter Volume } \\
& MS $(\mathbf{n}=\mathbf{2 3})$ & Control $^{\mathbf{b}}(\mathbf{n}=\mathbf{2 4})$ \\
\hline Strenuous Physical Activity & $0.442^{*}$ & $\mathbf{0 . 5 3 1}$ \\
Neuropsychological Composite & 0.061 & -0.032 \\
Cognitive Efficiency & -0.041 & 0.013 \\
Average Fractional Anisotropy & -0.067 & 0.146 \\
Average Axial Diffusivity $^{\mathbf{c}}$ & 0.196 & -0.038 \\
Average Radial Diffusivity $^{\mathbf{c}}$ & 0.098 & -0.156 \\
\hline
\end{tabular}

${ }^{*} p \leq 0.1$ (trend); ${ }^{* *} p<0.01 ; * * * p<0.001$

${ }^{a}$ r-value reported

${ }^{\mathrm{b}}$ Log-transformed

${ }^{c}$ Entire white matter skeleton 


\subsection{Discussion.}

The purpose of this study was to examine the relation between physical activity, white matter integrity and cognitive efficiency in pediatric-onset multiple sclerosis. As expected, differences in diffusion parameters of the white matter skeleton were observed between groups, with patients consistently exhibiting indices of lower white matter integrity. Total T1 and T2 lesion volumes correlated with several white matter tracts. Associations between white matter integrity and cognitive composite scores were not observed. While self-reported strenuous physical activity did not correlate with diffusion parameters of white matter integrity, it was significantly associated with normalized white matter volume in controls, and demonstrated a trend toward a positive association with white matter volume in patients.

\subsection{Sample Characteristics}

Similar to previous studies, our sample of pediatric-onset MS patients was comparable to other study samples in the literature with respect to disease duration, disability, and relapse rate (Amato et al., 2009; MacAllister, Boyd, Holland, Milazzo, \& Krupp, 2007; Till et al., 2012). Patients and controls did not differ in age, sex ratio, years of education, or SES. Inconsistent with previous literature (Amato et al., 2010; Grover et al., 2015), the current sample of patients did not differ from controls with respect to mood, fatigue or level of self-reported strenuous physical activity. Given that increased physical activity is known to impart positive effects on both mood

and fatigue (Babyak et al., 2000; Blumenthal et al., 2009; Jerstad, Boutelle, Ness, \& Stice, 2010), it is possible that higher levels of physical activity contributed to the similarities noted between patients and controls in this study.

Notably, groups differed with respect to vocabulary, fine motor dexterity, and IQ. This is consistent with previous work conducted in independent samples of pediatric-onset MS patients (Till et al., 2011; Till et al., 2013). It should be noted, however, that even though MS patients scored lower on these measures, they still fell within the age-expected range. As such, this sample of MS patients is relatively cognitively intact in comparison to previous studies (Amato et al., 2009; Till et al., 2011).

Finally, while lesion volume was quite variable among patients, they were comparable to what has been observed in other pediatric-onset MS samples (Till et al., 2011, 2012). Moreover, MS patients demonstrated smaller normalized thalamic volume and normalized white matter volume. Previous research has noted that thalamic volume is sensitive to disease pathology in 
pediatric onset MS (Mesaros et al., 2008; Till et al., 2011, 2012), though there is less consensus with regards to the clinicopathological relevance of white matter volume (Till et al., 2011; Tortorella et al., 2006). While it is generally understood that white matter is negatively impacted by inflammation due to MS disease activity, the impact on the thalamus may reflect Wallerian degeneration, given the number of white matter projections originating from this structure (Mesaros et al., 2008). Although slightly lower grey matter volume was noted in patients, groups did not differ significantly in this MRI metric. This finding may reflect a lack of significant secondary disease mechanisms, such as Wallerian degeneration, in the grey matter of this sample, however this conclusion is purely speculative.

\subsection{Group differences in diffusion parameters}

The first aim of this study was to examine differences in white matter integrity between pediatric-onset MS patients and healthy controls, using tract-based spatial statistics (TBSS). This method for examining diffusion tensor imaging data has been validated as a reliable measure for investigating whole brain changes in white matter microstructure (Smith et al., 2006).

In accordance with the study's hypothesis, differences in diffusion parameters were noted between patients and controls, with patients exhibiting indices of lower white matter integrity. Specifically, lower FA in the entire white matter skeleton, as well as in several white matter tracts was noted in patients. FA is the primary index of white matter integrity in DTI analyses, and provides an indirect measure of myelination (Feldman et al., 2010). Indeed, previous studies have noted that the FA of myelinated and non-myelinated fibres differ significantly, with lower FA in lesions compared to normal-appearing white matter (NAWM; Sbardella, Tona, Petsas, \& Pantano, 2013; Werring, Clark, Barker, Thompson, \& Miller, 1999). The findings of this study are consistent with previous literature which has noted decreased FA in pediatric-onset MS patients (Absinta et al., 2010; Akbar et al., 2016; Blaschek et al., 2013; Rocca, Valsasina, et al., 2014; Rocca, Absinta, et al., 2014; Tillema et al., 2012; Vishwas et al., 2013; Vishwas, Chitnis, Pienaar, Healy, \& Grant, 2010). Similar to Akbar et al., (2016) significantly lower FA values were observed in the subfields of the corpus callosum, the posterior thalamic radiation, the sagittal stratum, and the superior/posterior corona radiata. The aforementioned tracts are all major white matter tracts that may be vulnerable to disease pathology, given their proximity to areas of the brain with high lesional probability (e.g., periventricular regions; Yeh et al., 2009). Notably, previous DTI studies have observed that the corpus callosum (i.e., the major white 
matter structure in the brain connecting the cerebral hemispheres) is particularly impacted by MS disease pathology (Akbar, Giorgio, et al., 2016; Blaschek et al., 2013; Rocca, Absinta, et al., 2014; Tillema et al., 2012; Vishwas et al., 2013). Moreover, given that lesions were not removed in these analyses to examine NAWM, the FA values extracted may have been impacted by the presence of lesions in these tracts.

This study noted additional reductions in the FA values of the anterior corona radiata, cerebral peduncle, retrolenticular part of the internal capsule, external capsule, and fornix, suggesting that widespread damage of white matter is present early in the disease course. This is consistent with previous studies which have observed lower FA in more diffuse white matter tracts of the brain, including the posterior limb of the internal capsule, the cerebral peduncle, and association fiber system (including the fornix and the superior longitudinal fasciculus; Vishwas et al., 2010; 2013). The extent of FA reductions observed in patients in this study highlights the sensitivity of the TBSS technique in detecting MS disease pathology.

Consistent with Akbar et al., (2016) greater RD in the entire white matter skeleton was noted in patients, relative healthy controls. In addition, greater AD was observed in patients, contrary to Akbar et al., (2016) who did not note a group difference in this metric. As mentioned previously, higher $\mathrm{AD}$ and $\mathrm{RD}$ values are indicative of axonal loss (Aung, Mar, \& Benzinger, 2013; Song et al., 2002) and demyelination (Klawiter et al., 2011), respectively. The discrepancy between this analysis and that conducted by Akbar et al., (2016) suggests that reduced white matter integrity in this population is not purely explained by demyelination, but that axonal loss is an additional contributing factor. Postmortem examination of the MS brain has noted decreased axonal density in lesioned tissue (Van Waesberghe et al., 1999), though notable exceptions exist (Schmierer et al., 2007).

It is important to acknowledge that of the aforementioned studies, different methods for investigating differences in white matter integrity were employed (i.e., voxel-based morphometry, tractography, and TBSS). Given the heterogeneity in how these different methods analyze DTI data, comparisons between studies employing different measures should be drawn with caution. Further examination of the differences between such methods will be explored in subsequent sections of this discussion. 


\subsubsection{White matter integrity and lesion volume}

It should be further noted that several studies also observed lower FA values in NAWM of pediatric-onset MS patients when compared to healthy controls (Bethune et al., 2011; Longoni et al., 2017; Till et al., 2011; Tillema et al., 2012; Tortorella et al., 2006; Vishwas et al., 2013, 2010a). According to the current results, it appears that differences in white matter integrity between patients and controls may be associated with lesion volume.

Indeed, higher T1- and T2-lesion volume correlated with lower FA in many tracts in which group differences were also noted (though some were trends). These included, the body and splenium of the corpus callosum; the bilateral anterior, posterior, and superior corona radiata; the bilateral posterior thalamic radiation; and the bilateral sagittal stratum. In adult MS, previous studies have co-localized FA results with lesion maps and have noted lower FA in focal white matter lesions defined by T2-weighted images (Bammer et al., 2000; Roosendaal et al., 2009). FA has been noted as a more sensitive metric for detecting tissue damage than MD, as its values have been shown to decrease according to the severity of tissue disruption (Bammer et al., 2000), and FA reductions in the corpus callosum and corona radiata have been associated with T2 lesion volume (Roosendaal et al., 2009). This study noted stronger associations between T1lesion volume and FA, compared to T2 lesion volume. This is consistent with previous research which has noted the most pronounced alterations in diffusion parameters in T1 hypointense lesions, given that they are characterized by more severe tissue injury (Bammer et al., 2000; Droogan et al., 1999; Filippi, 2001; Rocca, Cercignani, Iannucci, Comi, \& Filippi, 2000).

$\mathrm{T} 1$ and T2 lesion volumes were also negatively correlated with FA in the entire white matter skeleton, and showed a positive association with RD, and a trend for a positive association with AD. Indeed, while FA has been shown to be more sensitive to pathological damage than MD ( i.e., an index of myelin loss comprising AD and RD; Scanderbeg et al., 2000; Werring et al., 1999), both FA and MD are impacted by T2 lesions in adult MS (Cercignani et al., 2000; Christiansen et al., 1993; Droogan et al., 1999; Filippi et al., 2000; Larsson, Thomsen, Frederiksen, Stubgaard, \& Henriksen, 1992). Previous research examining diffusion parameters in pediatric-onset MS patients have also noted a relation between lesion burden and DTI abnormality (Bethune et al., 2011). Specifically, greater total T2-lesion volume has been associated with lower FA in the NAWM of the corpus callosum and of the average supratentorial hemispheric NAWM (Bethune et al., 2011). In this study, both T1- and T2-lesion burden appear 
to be linked to the degree of DTI abnormality. This is consistent with previous studies in pediatric-onset MS (Bethune et al., 2011), though notable exceptions exist (Absinta et al., 2010).

In adult MS, it is accepted that lesion volume is poorly correlated with physical disability (Barkhof, 1999; Yeh et al., 2009). These findings reflect the limitations of examining lesion volume in MS. T2-weigthed images are non-specific, and cannot distinguish between lesions caused by inflammation, edema, or axonal loss (Zivadinov \& Bakshi, 2004). Moreover, active demyelination may be identified by contrast enhancement in T1-weighted images; however, such enhancement is not a reliable index of disease progression or permanent injury (Filippi et al., 1997; Lai et al., 1996). A critical limitation to examining lesion volume in MS is also that it is unable to specify whether high lesion volume is indicative of a very large lesion, or multiple diffuse lesions. Indeed, some patients with only modest lesion volumes will present with advanced neurologic disability, whereas patients with many or enlarging lesions may show minimal disability (Thompson et al., 1990). However, given these arguments, when T1 and T2 lesion volume were controlled for, no associations between FA and the cognitive composite scores were observed. As such, it is not likely that lesion volume had a strong effect on this association, but rather that the structural damage observed in our sample was not sufficiently severe to produce a more profound impact on cognition.

\subsection{Indices of white matter integrity not associated with cognitive measures, MRI metrics}

Inconsistent with previous research, this study did not observe any associations between indices of white matter integrity and the cognitive composite scores, though a trend was observed between higher FA of the body of the corpus callosum and higher cognitive efficiency composite in the MS group. In adult MS, several studies have noted that tract injury identified by abnormal diffusion parameters is associated with cognitive dysfunction (Dineen et al., 2009; Hulst et al., 2013; Rovaris et al., 2002; Sbardella et al., 2013; Yu et al., 2012). In pediatric-onset MS, altered diffusion parameters, particularly lower FA in the corpus callosum, have been associated with reduced attentional control (Todorow, DeSouza, Banwell, \& Till, 2014), slower processing speed (Bethune et al., 2011), and poorer math performance (Till et al., 2011). It is worth noting that in two of the aforementioned studies that observed an association between cognitive measures and DTI abnormalities of the corpus callosum, only NAWM was examined (Bethune et al., 2011; Till et al., 2011). In the current study, lesions were included in the analyses, as previous research has demonstrated that the extent of $\mathrm{T} 2$ lesions in white matter 
tracts had more power in identifying neuropsychological impairment, over and above NAWM (Mesaros et al., 2012). Moreover, none of the aforementioned pediatric studies examined white matter integrity via the TBSS technique; rather, tractography and ROI-based methods were employed. However, there is evidence in the adult MS literature of an association between abnormal diffusion parameters analyzed via TBSS and cognitive dysfunction (Dineen et al., 2009; Hulst et al., 2013; Yu et al., 2012). As noted in the pediatric-onset population, the strongest associations have been observed in the posterior thalamic radiation, the sagittal stratum, and the corpus callosum (Yu et al., 2012), with lower FA related to slower processing speed (Dineen et al., 2009; Yu et al., 2012) as well as poorer visual and verbal learning and memory (Dineen et al., 2009).

Based on the literature in adult MS, TBSS appears to be an appropriate technique for identifying associations between white matter integrity and cognitive dysfunction. It should be noted, however, that the sample of MS patients in this study was relatively cognitively intact, which may have also masked any relations between the cognitive outcomes of interest and the diffusion parameters. Notably, Hulst et al., (2013) observed that the FA skeleton in cognitively preserved adult MS patients was affected to a lesser degree than in the cognitively impaired patients (49\% versus $76 \%$ of white matter affected, respectively). In cognitively-preserved pediatric MS patients, the white matter damage may not be sufficiently extensive to demonstrate a relation with cognitive ability. Perhaps if this sample were followed over time, the association would become more pronounced. Schoonheim, Meijer, \& Geurts (2015) propose a hypothesis for the development of cognitive impairment in MS. They note that in the early stages of MS, structural damage is low, allowing for neural networks to continue functioning efficiently. As such, patients remain cognitively intact for some time. However, over time, as structural damage accumulates, networks become less efficient, and eventually, a threshold is reached and it is likely that cognitive impairment ensues (Figure 3). 


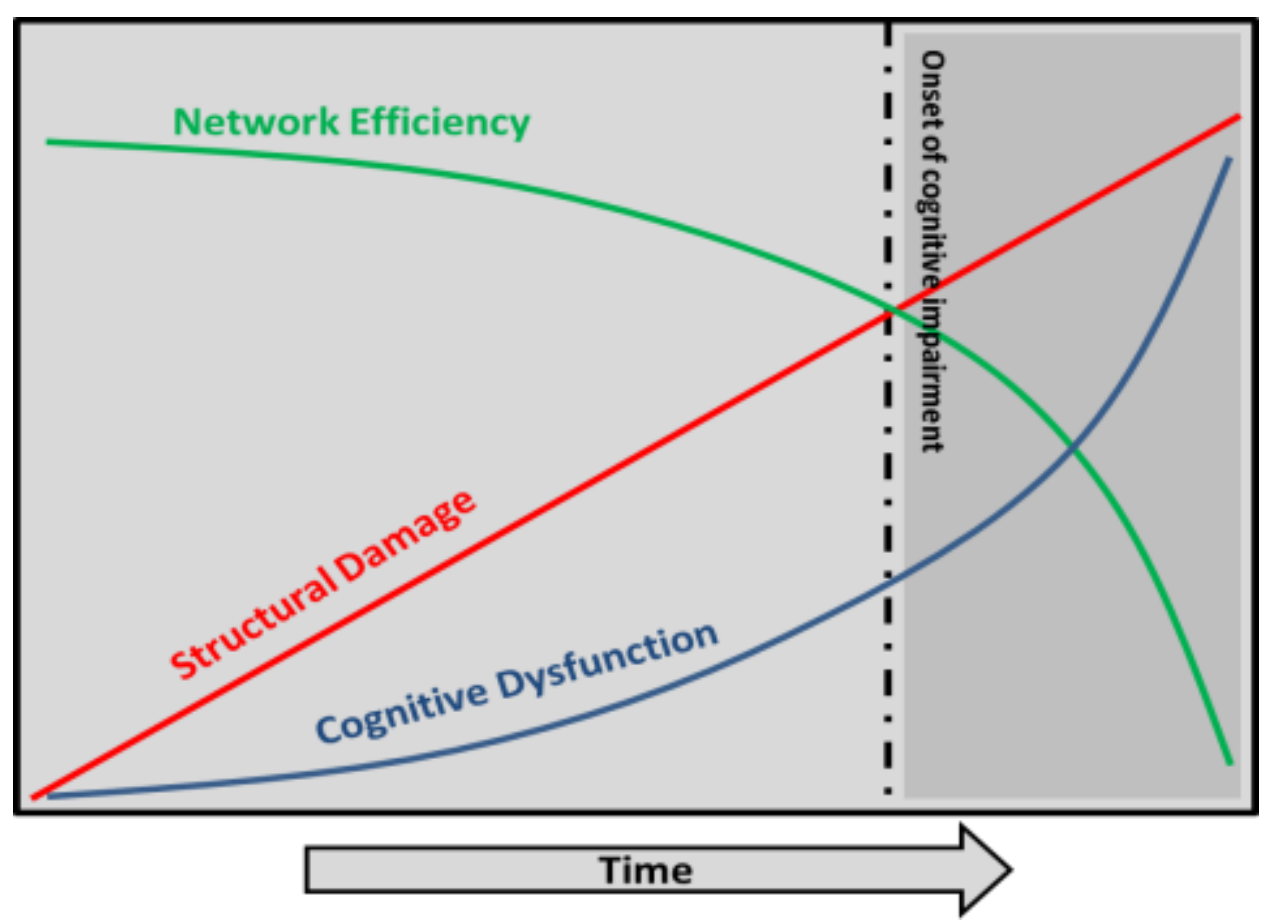

Figure 3. Hypothesis for the development of cognitive impairment in MS. Figure as originally published in Schoonheim, Meijer \& Geurts (2015), Front Neurol, 6:82.

Given that the patients in this sample are early in the stages of MS, it is probable that the structural damage caused by the disease is not yet significant enough to produce any negative impact on cognition. These patients are likely able to compensate for structural damage through the reorganization of neural networks necessary for cognitive processes. Rocca et al. (2015) noted that the structural damage contributing to cognitive impairment in pediatric-onset MS is likely driven by white matter damage, rather than cortical lesion volume or number. Indeed, they observed that while cognitively preserved and cognitively impaired pediatric-onset MS patients did not differ in terms of lesion volume/number, cognitively impaired patients had significantly lower white matter volume. Moreover, in another study, the same group observed that while cognitively preserved pediatric onset MS patients showed no DTI abnormalities compared to controls, the cognitively impaired group (defined by scoring greater than two standard deviations below the mean on two or more neuropsychological tests) demonstrated a diffuse pattern of decreased FA and increased RD in the corpus callosum, cingulum, fornix, and parieto-occipital white matter (Rocca et al., 2014). This study also noted functional connectivity differences between groups, such that cognitively impaired patients showed decreased functional connectivity of the right precuneus, whereas cognitively preserved patients demonstrated 
increased functional connectivity of the right anterior cingulate cortex. This is consistent with Akbar et al. (2016) who noted increased functional connectivity of the precuneus in cognitively preserved patients relative to healthy controls, particularly in connections with the anterior cingulate cortex, frontal medial cortex, and cerebellum, though not all studies have observed differences in resting state functional connectivity between cognitively impaired and cognitively preserved patients (Rocca, Valsasina, et al., 2014).

In line with the hypothesis proposed by Schoonheim, Meijer \& Geurts (2015), these studies suggest that, despite structural damage, cognitively preserved patients may be recruiting compensatory mechanisms to maintain cognitive functioning. Such studies highlight the pivotal role that white matter damage plays in the pathogenesis of cognitive impairment in these patients, and demonstrate that the severity of such impairment may be more associated with posterior regions of the brain, where structural damage is more pronounced. Given these findings, the widespread structural pathology which was observed in this study through DTI may have been counteracted by the reorganization of networks and the use of compensatory mechanisms (e.g., increased functional connectivity). As such, the association between structural pathology and cognition may have been masked, and patients presented as relatively cognitively intact.

Nonetheless, trends were noted in MS patients indicating that lower FA of the bilateral external capsule and the right superior longitudinal fasciculus associated with higher neuropsychological composite scores. A pattern of negative association between other MRI metrics (i.e., grey/white matter volume and brain volume) were observed with FA of the corona radiata and the internal capsule. Indeed, it does not seem logical that increased volume in other MRI metrics should associate with decreased white matter integrity, nor that lower FA would associate with stronger neuropsychological function. However, when interpreting potentially spurious results, it is important to consider the limitations of the TBSS analysis. Of note, TBSS makes use of the FA map and discards orientation information captured in the diffusion data; this creates complications in pathways where different structures merge, such as in the corona radiata (Bach et al., 2014). Moreover, tract specificity of the projection step in the analysis is not fully understood (Bach et al., 2014). Indeed, there is a body of literature that exists which cautions against over-interpretation of associations (especially if they are at the trend level) that are noted with specific tracts identified via TBSS (Bach et al., 2014; Leow et al., 2009; Vos, Jones, 
Jeurissen, Viergever, \& Leemans, 2012). Such limitations may have also contributed to the lack of association observed between self-reported strenuous physical activity and diffusion parameters.

\subsection{Self-reported physical activity is not associated with diffusion parameters, but is correlated to white matter volume}

Contrary to the hypotheses, diffusion parameters did not correlate with self-reported strenuous physical activity in either pediatric-onset MS patients or healthy controls. This is inconsistent with previous literature in healthy children and adolescents which has noted that higher-fit individuals have greater white matter integrity, particularly of the corpus callosum (Chaddock-Heyman et al., 2014). However, even within healthy populations, there is limited research examining the impact of physical activity on white matter integrity in children and adolescents, and most of this research has been conducted in older and aging adults. While the beneficial effects of exercise on white matter integrity have been noted in the adult MS population (Dalgas \& Stenager, 2012; De Giglio et al., 2016; Ibrahim et al., 2011; Prakash, Patterson, Janssen, Abduljalil, \& Boster, 2011; Prosperini et al., 2014; Prosperini, Piattella, Giannì, \& Pantano, 2015; Rasova et al., 2015), little is known with regard to the effect of exercise on the brain in the pediatric population.

Notably, Grover et al. (2015) observed that pediatric-onset MS patients participated in less vigorous physical activity than healthy age- and sex-matched controls, and that increased physical activity in the MS group was associated with decreased lesion volume. In the current study sample, no between group differences in self-reported strenuous physical activity were noted. Moreover, the range of self-reported strenuous physical activity was large in the MS population (0-7 times/week), with no consistent pattern between level of physical activity and FA values. This observation highlights the limitations in conducting a cross-sectional study of this nature. For example, it is unclear whether the MS patients who reported participating in more strenuous physical activity have been doing so for a long time, and as such have higher FA in the tracts of interest, or whether they have just recently begun exercising more regularly, and subsequently still demonstrate lower FA values.

Self-reported strenuous physical activity also did not associate with the cognitive composite scores in either group. Previous research has noted that acute and chronic exercise regimens may exert different effects on executive functioning, given that most research 
examining these constructs have studied the positive effects of acute exercise on higher-level cognitive processes, but very few have investigated the impact of a chronic exercise regimen (Verburgh, Konigs, Scherder, \& Oosterlaan, 2013). Additionally, not all forms of exercise are thought to have equivalent benefits on brain health. For instance, Diamond (2015) notes that for physical activity to have an effect on children's executive functions, it must be cognitivelyengaging (e.g., sports that involve strategy, such as basketball). Moreover, studies examining the effect of exercises that involve character development (e.g., traditional martial arts) or mindfulness (e.g., yoga) are more effective in improving children's executive functions than exercise alone (e.g., running; Lakes \& Hoyt, 2004; Manjunath \& Telles, 2001). The current study did not gather data on the type of physical activity that participants were engaging in. Such information would have utility in future research in evaluating the effectiveness of physical activity on cognition in this population. In addition, physical activity was not associated with decreased lesion volume in this study, contrary to previous research (Grover et al., 2015). Methodological limitations may have been responsible for masking the expected result, such as insensitivity of the physical activity measure, or low power.

However, partial correlations controlling for age revealed a pattern in the corpus callosum in healthy controls that was not evident for MS patients. Specifically, FA of all subfields of the corpus callosum positively associated with greater levels of self-reported strenuous physical activity. Additionally, increased levels of physical activity were associated with lower RD in the entire white matter skeleton. These patterns were not observed in MS patients, suggesting that strenuous physical activity may have a differential effect on brain structure in patients and controls. Trends in the internal capsule were noted for MS patients; however, they demonstrated an inconsistent pattern, with a positive association noted between FA of the right anterior limb and strenuous physical activity, and a negative association with the FA of the right posterior limb. Such inconsistent results call into question the reliability of these findings. It should also be emphasized that these patterns were non-significant, further cautioning against over-interpretation of the results.

In controls, a consistent and significant positive association was noted between physical activity and normalized white matter volume. This relation was observed in MS patients only when analyses were not covaried for age. As such, it appears as though an association between 
physical activity and white matter exists, though it is driven by age in the MS group, and that this association is specific to white matter volume rather than white matter microstructure.

Indeed, the findings of the current study call into question the utility of the TBSS method in identifying associations between diffusion parameters and lifestyle factors. In previous literature, spurious correlations between improvement in motor performance and lower FA in several white matter tracts have been observed (Taubert et al., 2010). Taubert et al. (2010) employed the VBM method for extracting indices of white matter integrity following two sessions of practice on a balancing task. Contrary to expectations, decreases in FA were noted in association with learning-related improvements in motor performance. Upon further investigation, it was observed that the regions which showed such negative correlations were also areas of the brain where evidence for crossing fibers was observed (i.e., left prefrontal and right parietal white matter regions). As such, the negative correlations that were noted may be a product of the limitations of DTI analyses, rather than a representation of a true, meaningful association.

Previous research has noted that FA correlates with other MRI metrics including white matter volume (Kochunov et al., 2007), though there is limited research that has examined this relation. Indeed, there is an even greater literature that critiques DTI as a method, particularly the TBSS technique. Moreover, given that MS is an illness affecting white matter, special consideration into the limitations of this technique, specifically within this clinical population, is warranted. Identifying such limitations may provide insight as to why the expected associations were not observed in this study.

\subsection{Methodological considerations}

\subsubsection{Is DTI an accurate measure of white matter integrity?}

Since its conception, DTI has grown in popularity, particularly for evaluating disease pathology in illnesses of the brain. However, several studies have highlighted the limitations to this technique, the questions it can and cannot answer, and where it fails as a method (Assaf \& Pasternak, 2008; Leow et al., 2009; Sbardella et al., 2013; Vos et al., 2012). While anisotropy measures do change significantly when myelin is damaged or absent (such as in MS), the exact contribution of myelin to the DTI signal cannot be fully determined (Assaf \& Pasternak, 2008). Indeed, FA values will show a similar pattern of reduction in demyelination, edema, gliosis, and inflammation, suggesting that it is a non-specific metric. Moreover, the FA metric is confounded 
by the fact that it is a measure of several factors, including the directionality of individual fibres (which may be influenced by myelin thickness), as well as the number of dominant fibre directions (i.e. the density of fibres; Leow et al., 2009). This begs the question: is myelin a crucial factor for obtaining anisotropy measures from the DTI signal? The answer to this question remains largely unclear.

The assumptions of the DTI model also contribute to its limitations. The major assumption is that a single diffusion tensor is sufficient to characterize each voxel (Assaf \& Pasternak, 2008). In this study, a voxel size of $2 \times 2 \times 3 \mathrm{~mm}^{3}$ was employed. Tens of thousands of axons likely make up each voxel, and it is unlikely that a single diffusion tensor is representative of them all. As a result, the tensor is an average of the fibres in each voxel. Specifically, the DTI model fails in regions where the tensor averages areas where white/grey matter or white matter/CSF are found in the same voxel (termed "tissue partial volume"), or areas where white matter fibres cross in the same voxel (termed "white matter partial volume; Assaf \& Pasternak, 2008; Leow et al., 2009; Vos et al., 2012). Some studies have noted that diffusion parameters are adequate for modelling tracts with a single dominant orientation (e.g., the corpus callosum), but that it is not suitable for more complex fibres (e.g., the corona radiata; Gunnarsson, 2014). This calls into question the interpretability of diffusion parameters in a variety of white matter tracts, especially in clinical populations where white matter is affected, such as MS.

\subsubsection{Are diffusion parameters an accurate index of MS pathology?}

Indeed, diffusivity patterns differ according to MS phenotype and within different lesions and tissue types (Sbardella et al., 2013). A variety of methodological approaches have been employed to study diffusion parameters in MS, and to study different types of tissue damage. DTI has proven to be useful for detecting areas of demyelination in MS that are undetected by conventional MRI (Sbardella et al., 2013), and post-mortem research in MS has confirmed the association between diffusion parameters and histologically-derived measures of tract myelination (Kolasinski et al., 2012; Schmierer et al., 2007). Moreover, many studies have concluded that FA, $\mathrm{AD}$, and $\mathrm{RD}$ are good measures for disease pathology (Prosperini et al., 2014).

As mentioned previously, many studies have examined cortical NAWM, and have detected widespread DTI abnormalities in this tissue type, such as decreased FA and increased MD, in both adult MS (Bammer et al., 2000; Cercignani et al., 2000; O Ciccarelli, Werring, 
Barker, Thompson, \& Miller, 2001; Droogan et al., 1999; Filippi, 2001; Filippi et al., 2000; Guo, Jewells, \& Provenzale, 2001; Rocca et al., 2000), and in pediatric-onset MS (Bethune et al., 2011; Longoni et al., 2017; Till et al., 2011; Tillema et al., 2012; Tortorella et al., 2006; Vishwas et al., 2013, 2010a). Moreover, one study noted an FA gradient in adult MS patients, with lower FA values closer to lesions, and higher values further from the lesions (Guo et al., 2001). Most studies have noted that the degree of damage to NAWM varies according to the severity of pathology and as the disease worsens over time (Sbardella et al., 2013). Notably, Longoni et al., (2017) observed that over a median follow-up period of 4.4 years, pediatric-onset MS patients failed to show age-expected white matter development and a progressive loss of tissue integrity (indexed by decreases in FA and increases in MD of NAWM over time). The current study did not examine diffusion parameters in NAWM, as the impact of lesioned tissue on cognitive ability was of interest. Interestingly, the examination of NAWM in MS has been critiqued, with some suggesting that it may have limited value early on in the disease process, as the effects of the disease may be less pronounced (Sbardella et al., 2013).

Given the consistent results noted in the literature examining diffusion parameters in MS, it appears as though this is a valid method for investigating MS disease pathology. However, these findings do not acknowledge several limitations that arise in DTI analysis. The remainder of this discussion will examine the limitations of DTI analysis in MS, particularly with the TBSS method, which was employed in this study.

\subsubsection{Limitations of TBSS}

TBSS is the most popular approach for performing voxel-wise DTI analyses. This technique was developed to address alignment-related problems associated with voxel-based morphometry (VBM) and tractography, and as a method that could investigate the entire brain (as opposed to pre-delineated tracts of interest) in a fully automated manner (Smith et al., 2006). VBM is limited by alignment inaccuracies and smoothing extent, and tractography methods are limited in terms of finding the principal direction of a tract (i.e., in locations where there are crossing fibers, low signal-to-noise ratio and poor spatial resolution, small non-detectable fibers, and other distortions in the brain; Smith et al., 2006, 2007). TBSS was designed to overcome these problems in order to improve the sensitivity, objectivity, and interpretability of the results.

A major concern of TBSS analysis is the ongoing debate regarding its anatomical specificity (Bach et al., 2014). Since TBSS discards orientation information and makes use only 
of the FA map when projecting onto the FA skeleton, complications arise in terms of distinguishing between adjacent, but differentially oriented fibre bundles with similar FA values; for example, between the body of the corpus callosum and the corona radiata (Bach et al., 2014). Because the TBSS technique discards the orientation information, it becomes difficult to distinguish between such tracts, and as a result, the skeletonization process will cause these fibres to collapse onto one another. Bach et al. (2014) noted significant group differences in FA in regions where voxel misalignment had occurred, and that small tracts were more susceptible to such errors (e.g., the fornix). Given that the threshold-free cluster enhancement (TFCE) correction depends on statistical support from adjacent voxels in order to detect group differences, voxel misalignment that causes structures to collapse onto one another or merge would dramatically alter the significance map. In addition to this, group differences were observed in noise-induced structures which were otherwise anatomically-meaningless, highlighting the risk of false positives in TBSS analyses. Finally, the authors examined the extent to which the results obtained through TBSS analyses depend on user-specified settings. They found that different results were obtained depending on the registration target used. In FSL, the options for registration targets are the FMRIB58 template (provided by the software), or a studyspecific target, where the most representative subject from the group is used as the registration target. The authors noted that a smoother FA map and a clearer depiction of white matter structures were obtained from registration to the FMRIB58 target, rather than the study-specific target. Indeed, a similar observation was noted for the data in the current study, and as a result, the FMRIB58 target was used.

While it has been validated as an accurate measure of white matter microstructure in MS, it is evident that the TBSS technique has its limitations. Additionally, based on these critiques, it is plausible that the spurious associations noted in the current study may have been due to alignment error, or a liberal statistical correction. Indeed, the regions which demonstrated unexpected patterns of association with the cognitive and MRI data are tracts that are smaller (i.e., the external capsule) and where tracts change in orientation (i.e., the corona radiata), or cross other tracts (i.e., the posterior thalamic radiation). Given that several unexpected negative associations were observed in this study, the interpretation of these results should be done with caution. As such, investigating tracts that are more robust against the limitations of TBSS analysis (i.e., corpus callosum, and large association fibres, such as the superior longitudinal 
fasciculus), should be emphasized more than those which are vulnerable to alignment error (i.e., projection fibres, such as the corona radiata and tracts originating from the thalamus). However, the between group results are consistent with the findings in published research that examined the same cohort of MS patients (Akbar et al., 2016). As such, TBSS-derived diffusion parameters identify some aspects of disease pathology, at least in major white matter tracts that are more vulnerable in MS (e.g., corpus callosum). It is also noteworthy that employing the TBSS technique provided information on whole brain white matter microstructure, and our analyses were not limited to specific tracts or voxels of interest (as in VBM and tractography analyses). Given the limitations outlined in this section, the group differences and associations with average $\mathrm{FA}, \mathrm{AD}$, and $\mathrm{RD}$ of the entire white matter skeleton are likely the most informative results of this study. Nevertheless, using multiple methods for measuring white matter pathology along with global white matter volume may provide a clearer understanding of the effect of MS on white matter microstructure, and its association with cognitive ability.

\subsubsection{White matter volume}

Indeed, the recent popularity in DTI analyses has been promising; however, each of the DTI techniques (i.e., VBM, tractography, TBSS) have several limitations which have been highlighted in the literature. In this study, the only significant association with physical activity was noted with white matter volume. Although some trends were noted with diffusion parameters in the healthy controls, this result should not be over-interpreted. While white matter volume is not as sensitive to the microstructural changes that occur early in the MS disease process, associations with white matter volume are less ambiguous than those with the diffusion parameters.

As described in the Methods Section, the SIENAX software was used to estimate normalized whole-brain grey matter and white matter volumes (Smith et al., 2002). This method defines brain size with respect to skull size, and is normalized to the MNI152 standard template. This procedure reduces within-group variability, making cross-sectional comparisons more sensitive (Smith et al., 2002). Additionally, the segmentation procedure estimates the partial volume effects at all voxels (i.e., to what extent a particular voxel contains more white or grey matter in areas of overlap), providing greater accuracy in identifying the different tissue types. This method has been validated, and the mean error in segmentation was determined to be $0.4 \%$ of brain volume, while the mean error in normalized brain volume (including white/grey matter 
volume) was $1 \%$ (Smith et al., 2002). As such, estimates of normalized white matter volume in this study are likely reliable and valid. Indeed, it appears as though a direct measure of white matter (such as white matter volume) may be more easily interpreted than diffusion parameters, which provide an indirect measure of white matter integrity.

The relation between white matter volume and white matter microstructure (i.e., diffusion parameters) remains to be fully described. As mentioned, while a positive association between FA and white matter volume has been noted (Kochunov et al., 2007), there is limited research that has examined this relation. Notably, Tamnes et al. (2010) observed that there were fewer and less significant correlations between white matter volume and diffusion parameters, than between white matter volume and cortical thickness. This study further noted that for half of the queried regions in the brain, white matter volume and white matter microstructure showed unique associations with age (i.e., brain maturation). In other words, these measures are not totally overlapping and may reflect different maturational processes in the brain. As such, examining both white matter volume and white matter microstructure is warranted.

Ultimately, while diffusion parameters were sensitive to disease pathology in our sample, their association with cognitive outcomes and physical activity were inconsistent, and thus difficult to interpret. A moderate association between white matter volume and physical activity was noted, highlighting the utility of including more direct measures of white matter in this population. In future studies, multiple methods for measuring white matter microstructure should be employed, in order to optimize the interpretability of the results. Such methods include other diffusion MRI techniques, as well as novel neuroimaging techniques for measuring white matter in the brain.

\subsubsection{Complementary MRI techniques for measuring white matter}

It may be of utility to compare diffusion parameters with other measures of myelin content in the future, such as myelin water imaging; an in-vivo technique which measures water content to identify different tissue types (Mackay \& Laule, 2012). This technique has been validated in post-mortem studies as accurately identifying myelinated axons in the brain (Mackay \& Laule, 2012). It has further been identified as an appropriate technique in MS research, and is able to distinguish between lesions based on age, as well as differentiate between NAWM in healthy controls versus adult MS patients (Laule et al., 2008). The utility of myelin water imaging should be investigated in pediatric-onset MS, as it may provide a complementary 
method to DTI analysis for distinguishing white matter abnormalities in this population. Moreover, other diffusion MRI techniques which estimate the microstructural features of dendrites and axons, such as neurite orientation dispersion and density imaging (NODDI) should be conducted in conjunction with standard DTI analyses. NODDI is a novel technique which estimates the orientation and density of neurites, both of which are contributing factors to FA (Zhang, Schneider, Wheeler-Kingshott, \& Alexander, 2012). As such, this method allows for density and orientation to be analyzed individually, and thus provides a more specific marker of brain integrity than standard DTI analyses. The combination of various indices of white matter integrity should be considered for future research in pediatric-onset MS.

\subsection{Limitations and Future Directions}

Despite the limitations of DTI analyses cited in this discussion, further investigation into its utility in pediatric-onset MS is warranted, specifically in terms of TBSS. As mentioned above, the use of multiple measures of white matter integrity should be employed in future research (i.e., myelin water imaging, NODDI). The combination of such measures of white matter microstructure, in addition to standard DTI analysis, may have utility in identifying early signs of demyelination, and may be more sensitive to disease pathology.

This study was further limited by the sample being relatively cognitively intact in comparison to previously-studied pediatric-onset MS cohorts. Further research investigating the association between white matter abnormalities, cognitive ability, and physical activity are warranted in this population. A longitudinal design is needed in the future to investigate the longterm effect of exercise on cognition and white matter. Moreover, collection of the physical activity measure was limited in that total minutes for each bout of exercise were not collected. Future research should ensure that this information is gathered in order to estimate the amount of time that participants are engaging in physical activity. Furthermore, a direct and objective measure of physical fitness is warranted in future research. As self-report questionnaires are prone to bias, complementing this data with measures such as $\mathrm{VO}_{2}$-max or accelerometry would provide a more accurate estimate of fitness.

\subsection{Conclusions}

The proposed study aimed to elucidate the relationship between physical activity, white matter integrity, and cognitive efficiency in patients with pediatric-onset MS. 
This study noted that diffusion parameters were sensitive to disease pathology, as MS patients consistently demonstrated lower indices of white matter integrity compared to healthy controls. Moreover, the white matter tracts which significantly differed between patients and controls also demonstrated strong associations with both T1 and T2 lesion volumes. This study did not confirm the hypotheses that diffusion parameters would be associated with cognitive outcomes and self-reported strenuous physical activity. It was noted, however, that white matter volume was positively associated with the physical activity measure in controls, and trended for a positive association in MS. The potential limitations in the sensitivity of TBSS-derived diffusion parameters to identify associations with lifestyle and cognitive outcomes was discussed. Notably, this study has highlighted the inconsistencies that exist regarding the methods employed for conducting DTI analyses to evaluate such associations. Future research should aim to use multiple methods for evaluating indices white matter integrity and their associations with cognition and physical activity.

Ultimately, physical activity was noted to be associated with white matter volume. This relation, as well as relations with other indices of white matter integrity should continue to be investigated in both healthy children and adolescents, as well as in the pediatric-onset MS population, as little research exists in this regard. Moreover, longitudinal studies may provide insight as to whether physical activity may be associated with greater white matter microstructure and contribute to improved cognitive efficiency in pediatric-onset MS patients. Demyelination resulting from MS occurs during a critical time when neural networks are developing in children. Given the young age of pediatric-onset MS patients, impairment in cognition may be due to the abnormal development of major white matter pathways that are implicated in cognitive ability. As such, continued investigation of modifiable lifestyle factors (such as physical activity) that may moderate disease-related neuroinflammation and cognitive decline is warranted. 


\subsection{References}

Absinta, M., Rocca, M. a, Moiola, L., Ghezzi, A., Milani, N., Veggiotti, P., ... Filippi, M. (2010). Brain macro- and microscopic damage in patients with paediatric MS. Journal of Neurology, Neurosurgery, and Psychiatry, 81(12), 1357-1362.

http://doi.org/10.1136/jnnp.2010.205682

Akbar, N., Giorgio, A., Till, C., Sled, J. G., Doesburg, S. M., De Stefano, N., \& Banwell, B. (2016). Alterations in functional and structural connectivity in pediatric-onset multiple sclerosis. PLoS ONE, 11(1), 1-14. http://doi.org/10.1371/journal.pone.0145906

Akbar, N., Till, C., Sled, J. G., Binns, M. a, Doesburg, S. M., Aubert-Broche, B., ... Banwell, B. (2016). Altered resting-state functional connectivity in cognitively preserved pediatric-onset MS patients and relationship to structural damage and cognitive performance. Multiple Sclerosis (Houndmills, Basingstoke, England), 22(6), 792-800.

http://doi.org/10.1177/1352458515602336

Amato, M., Goretti, B., Ghezzi, A., Lori, S., Zipoli, V., Moiola, L., ... Trojano, M. (2009). Cognitive and psychosocial features of childhood and juvenile MS. Neurology, 72(13), 1189.

Amato, M. P., Goretti, B., Ghezzi, A., Lori, S., Zipoli, V., Moiola, L., ... Multiple Sclerosis Study Group of the Italian Neurological, S. (2010). Cognitive and psychosocial features in childhood and juvenile MS: two-year follow-up. Neurology, 75(13), 1134-1140. http://doi.org/10.1212/WNL.0b013e3181f4d821; 10.1212/WNL.0b013e3181f4d821

Archer, T., Svensson, K., \& Alricsson, M. (2012). Physical exercise ameliorates deficits induced by traumatic brain injury. Acta Neurologica Scandinavica, 125(5), 293-302. http://doi.org/10.1111/j.1600-0404.2011.01638.x

Asato, M. R., Terwilliger, R., Woo, J., \& Luna, B. (2010). White matter development in adolescence: A DTI study. Cerebral Cortex, 20(9), 2122-2131. http://doi.org/10.1093/cercor/bhp282

Assaf, Y., \& Pasternak, O. (2008). Diffusion tensor imaging (DTI)-based white matter mapping in brain research: A review. Journal of Molecular Neuroscience, 34(1), 51-61. http://doi.org/10.1007/s12031-007-0029-0

Aubert-Broche, B., Fonov, V., Narayanan, S., Arnold, D. L., Araujo, D., Fetco, D., ... Collins, D. L. (2014). Onset of multiple sclerosis before adulthood leads to failure of age-expected 
brain growth. Neurology, 83(23), 2140-2146.

http://doi.org/10.1212/WNL.0000000000001045

Aung, W. Y., Mar, S., \& Benzinger, T. L. (2013). Diffusion tensor MRI as a biomarker in axonal and myelin damage. Imaging in Medicine, 5(5), 427-440. http://doi.org/10.2217/iim.13.49

Babyak, M., Blumenthal, J. a, Herman, S., Khatri, P., Doraiswamy, M., Moore, K., ... Krishnan, K. R. (2000). Exercise treatment for major depression: maintenance of therapeutic benefit at 10 months. Psychosomatic Medicine, 62(21), 633-638. http://doi.org/00006842200009000-00006

Bach, M., Laun, F. B., Leemans, A., Tax, C. M. W., Biessels, G. J., Stieltjes, B., \& Maier-Hein, K. H. (2014). Methodological considerations on tract-based spatial statistics (TBSS). NeuroImage, 100, 358-369. http://doi.org/10.1016/j.neuroimage.2014.06.021

Bammer, R., Augustin, M., Strasser-Fuchs, S., Seifert, T., Kapeller, P., Stollberger, R., ... Fazekas, F. (2000). Magnetic resonance diffusion tensor imaging for characterizing diffuse and focal white matter abnormalities in multiple sclerosis. Magnetic Resonance in Medicine, 44(4), 583-591. http://doi.org/10.1002/1522-2594(200010)44:4<583::AIDMRM12>3.0.CO;2-O

Banwell, B., Ghezzi, A., Bar-Or, A., Mikaeloff, Y., \& Tardieu, M. (2007). Multiple sclerosis in children: clinical diagnosis, therapeutic strategies, and future directions. The Lancet Neurology, 6(10), 887-902. http://doi.org/10.1016/S1474-4422(07)70242-9

Banwell, B. L., \& Anderson, P. E. (2005). The cognitive burden of multiple sclerosis in children. Neurology, 64, 891-894. http://doi.org/10.1212/01.WNL.0000152896.35341.51

Barkhof, F. (1999). MRI in multiple sclerosis: correlation with expanded disability status scale (EDSS). Multiple Sclerosis (Houndmills, Basingstoke, England), 5(4), 283-286. http://doi.org/10.1191/135245899678846221

Barnea-Goraly, N. (2005). White Matter Development During Childhood and Adolescence: A Cross-sectional Diffusion Tensor Imaging Study. Cerebral Cortex, 15(12), 1848-1854. http://doi.org/10.1093/cercor/bhi062

Barratt, W. (2005). The Barratt Simplified Measure of Social Status (BSMSS): Measuring SES. Terre Haute: Department of Educational Leadership, Administration, and Foundations, Indiana State University.

Benedict, R., Duquin, J., Jurgensen, S., Rudick, R., Feitcher, J., Munschauer, F., ... Weinstock- 
Guttman, B. (2008). Repeated assessment of neuropsychological deficits in multiple sclerosis using the Symbol Digit Modalities Test and the MS Neuropsychological Screening Questionnaire. Multiple Sclerosis (Houndmills, Basingstoke, England), 14(7), 940-6. http://doi.org/10.1177/1352458508090923

Bergendal, G., Fredrikson, S., \& Almkvist, O. (2007). Selective decline in information processing in subgroups of multiple sclerosis: an 8-year longitudinal study. European Neurology, 57(4), 193-202. http://doi.org/10.1159/000099158

Bethune, a., Tipu, V., Sled, J. G., Narayanan, S., Arnold, D. L., Mabbott, D., ... Banwell, B. (2011). Diffusion tensor imaging and cognitive speed in children with multiple sclerosis. Journal of the Neurological Sciences, 309(1-2), 68-74. http://doi.org/10.1016/j.jns.2011.07.019

Bitar, R., Leung, G., Perng, R., Tadros, S., Moody, A. R., Sarrazin, J., ... Roberts, T. P. (2006). MR Pulse Sequences: What Every Radiologist Wants to Know but Is Afraid to Ask. RadioGraphics, 26(2), 513-537. http://doi.org/10.1148/rg.262055063

Blaschek, a, Keeser, D., Muller, S., Koerte, I. K., Sebastian Schroder, A., Muller-Felber, W., ... Ertl-Wagner, B. (2013). Early White Matter Changes in Childhood Multiple Sclerosis: A Diffusion Tensor Imaging Study. AJNR Am J Neuroradiol, 34(10), 2015-2020. http://doi.org/10.3174/ajnr.A3581

Blumenthal, J., Babyak, M. A., Doraiswamy, P. M., Hoffman, B. M., Barbour, K. A., Herman, S., ... Sherwood, A. (2009). Exercise and pharmacotherapy in the treatment of major depressive disorder. Psychosomatic Medicine, 69(7), 587-596. http://doi.org/10.1097/PSY.0b013e318148c19a.Exercise

Briken, S., Gold, S. M., Patra, S., Vettorazzi, E., Harbs, D., Tallner, A., ... Heesen, C. (2014). Effects of exercise on fitness and cognition in progressive MS: a randomized, controlled pilot trial. Multiple Sclerosis (Houndmills, Basingstoke, England), 20(3), 382-90. http://doi.org/10.1177/1352458513507358

Bunge, S. A., Dudukovic, N. M., Thomason, M. E., Vaidya, C. J., \& Gabrieli, J. D. E. (2002). Immature frontal lobe contributions to cognitive control in children: Evidence from fMRI. Neuron, 33(2), 301-311. http://doi.org/10.1016/S0896-6273(01)00583-9

Bush, G., Luu, P., \& Posner, M. (2000). Cognitive and emotional influences in anterior cingulate cortex. Trends in Cognitive Sciences, 4(6), 215-222. http://doi.org/10.1016/S1364- 
6613(00)01483-2

Cardoso, M., Olmo, N. R., \& Fragoso, Y. D. (2015). Systematic Review of Cognitive Dysfunction in Pediatric and Juvenile Multiple Sclerosis. Pediatric Neurology, 53(4), 287292. http://doi.org/10.1016/j.pediatrneurol.2015.06.007

Cercignani, M., Iannucci, G., Rocca, M. a, Comi, G., Horsfield, M. a, \& Filippi, M. (2000). Pathologic damage in MS assessed by diffusion-weighted and magnetization transfer MRI. Neurology, 54(5), 1139-44. http://doi.org/10.1212/WNL.54.5.1139

Chaddock-Heyman, L., Erickson, K. I., Chappell, M. A., Johnson, C. L., Kienzler, C., Knecht, A., ... Kramer, A. F. (2016). Aerobic fitness is associated with greater hippocampal cerebral blood flow in children. Developmental Cognitive Neuroscience, Accepted M. http://doi.org/10.1016/j.den.2016.07.001

Chaddock-Heyman, L., Erickson, K. I., Holtrop, J. L., Voss, M. W., Pontifex, M. B., Raine, L. B., ... Kramer, A. F. (2014). Aerobic fitness is associated with greater white matter integrity in children. Frontiers in Human Neuroscience, 8(August), 584.

http://doi.org/10.3389/fnhum.2014.00584

Chaddock, L., Erickson, K. I., Prakash, R. S., Kim, J. S., Voss, M. W., Vanpatter, M., ... Kramer, A. F. (2010). A neuroimaging investigation of the association between aerobic fitness, hippocampal volume, and memory performance in preadolescent children. Brain Research, 1358, 172-183. http://doi.org/10.1016/j.brainres.2010.08.049

Chaddock, L., Pontifex, M. B., Hillman, C. H., \& Kramer, A. F. (2011). A Review of the Relation of Aerobic Fitness and Physical Activity to Brain Structure and Function in Children. Journal of the International Neuropsychological Society, 17(6), 975-985. http://doi.org/Doi 10.1017/S1355617711000567

Chard, D. T., Griffin, C. M., McLean, M. a, Kapeller, P., Kapoor, R., Thompson, a J., \& Miller, D. H. (2002). Brain metabolite changes in cortical grey and normal-appearing white matter in clinically early relapsing-remitting multiple sclerosis. Brain : A Journal of Neurology, 125(Pt 10), 2342-2352. http://doi.org/10.1002/ana.1123

Chiaravalloti, N. D., \& DeLuca, J. (2008). Cognitive impairment in multiple sclerosis. The Lancet Neurology, 7(12), 1139-1151. http://doi.org/10.1016/S1474-4422(08)70259-X Chitnis, T. (2013). Paediatric MS is the same disease as adult MS: No. Multiple Sclerosis Journal, 19(10), 1255-1256. http://doi.org/10.1177/1352458513488842 
Christiansen, P., Gideon, P., Thomsen, C., Stubgaard, M., Henriksen, O., \& Larsson, H. B. W. (1993). Increased water self???diffusion in chronic plaques and in apparently normal white matter in patients with multiple sclerosis. Acta Neurologica Scandinavica, 87(3), 195-199. http://doi.org/10.1111/j.1600-0404.1993.tb04100.x

Ciccarelli, O., Werring, D. J., Barker, G. J., Griffin, C. M., Wheeler-Kingshott, C. A. M., Miller, D. H., \& Thompson, A. J. (2003). A study of the mechanisms of normal-appearing white matter damage in multiple sclerosis using diffusion tensor imaging: Evidence of Wallerian degeneration. Journal of Neurology, 250(3), 287-292. http://doi.org/10.1007/s00415-0030992-5

Ciccarelli, O., Werring, D. J., Barker, G. J., Thompson, A. J., \& Miller, D. H. (2001). Investigation of MS normal-appearing brain using diffusion tensor MRI with clinical correlations. Neurology, 56, 319-320.

Compston, A., \& Coles, A. (2008). Multiple sclerosis. Lancet, 372(9648), 1502-17. http://doi.org/10.1016/S0140-6736(08)61620-7

Dalgas, U., \& Stenager, E. (2012). Exercise and disease progression in multiple sclerosis: can exercise slow down the progression of multiple sclerosis? Therapeutic Advances in Neurological Disorders, 5(2), 81-95. http://doi.org/10.1177/1756285611430719

De Giglio, L., Upadhyay, N., De Luca, F., Prosperini, L., Tona, F., Petsas, N., .. Pantano, P. (2016). Corpus callosum microstructural changes associated with Kawashima Nintendo Brain Training in patients with multiple sclerosis. Journal of the Neurological Sciences, 370, 211-213. http://doi.org/10.1016/j.jns.2016.09.041

DeLuca, J., Chelune, G. J., Tulsky, D. S., Lengenfelder, J., \& Chiaravalloti, N. D. (2004). Is speed of processing or working memory the primary information processing deficit in multiple sclerosis? Journal of Clinical and Experimental Neuropsychology, 26(4), 550-562. http://doi.org/10.1080/13803390490496641

Demaree, H. a, DeLuca, J., Gaudino, E. a, \& Diamond, B. J. (1999). Speed of information processing as a key deficit in multiple sclerosis: implications for rehabilitation. Journal of Neurology, Neurosurgery, and Psychiatry, 67(5), 661-663.

http://doi.org/10.1136/jnnp.67.5.661

Denney, D. R., Lynch, S. G., Parmenter, B. a, \& Horne, N. (2004). Cognitive impairment in relapsing and primary progressive multiple sclerosis: mostly a matter of speed. Journal of 
the International Neuropsychological Society, 10(7), 948-956.

http://doi.org/10.1017/S1355617704107030

Desouza, D. D., Hodaie, M., \& Davis, K. D. (2014). Abnormal trigeminal nerve microstructure and brain white matter in idiopathic trigeminal neuralgia. Pain, 155(1), 37-44.

http://doi.org/10.1016/j.pain.2013.08.029

Diamond, A. (2015). Effects of Physical Exercise on Executive Functions: Going beyond Simply Moving to Moving with Thought. Ann Sports Med Res, 2(1), 1011-1017. http://doi.org/10.1038/nbt.3121.ChIP-nexus

Dineen, R. A., Vilisaar, J., Hlinka, J., Bradshaw, C. M., Morgan, P. S., Constantinescu, C. S., \& Auer, D. P. (2009). Disconnection as a mechanism for cognitive dysfunction in multiple sclerosis. Brain, 132(1), 239-249. http://doi.org/10.1093/brain/awn275

Droogan, A. G., Clark, C. A., Werring, D. J., Barker, G. J., McDonald, W. I., \& Miller, D. H. (1999). Comparison of multiple sclerosis clinical subgroups using navigated spin echo diffusion-weighted imaging. Magnetic Resonance Imaging, 17(5), 653-661. http://doi.org/10.1016/S0730-725X(99)00011-9

Egan, M. F., Kojima, M., Callicott, J. H., Goldberg, T. E., Kolachana, B. S., Bertolino, A., ... Weinberger, D. R. (2003). The BDNF val66met polymorphism affects activity-dependent secretion of BDNF and human memory and hippocampal function. Cell, 112(2), 257-269. http://doi.org/10.1016/S0092-8674(03)00035-7

Erickson, K. I., Leckie, R. L., \& Weinstein, A. M. (2014). Physical activity, fitness, and gray matter volume. Neurobiology of Aging, 35(SUPPL.2), S20-S28. http://doi.org/10.1016/j.neurobiolaging.2014.03.034

Faulstich, M. E., Carey, M. P., Ruggiero, L., Enyart, P., \& Gresham, F. (1986). Assessment of depression in childhood and adolescence: an evaluation of the Center for Epidemiological Studies Depression Scale for Children (CES-DC). Am J Psychiatry, 143(8), 1024-1027.

Feldman, H. M., Yeatman, J. D., Lee, E. S., Barde, L. H. F., \& Gaman-Bean, S. (2010). Diffusion tensor imaging: a review for pediatric researchers and clinicians. Journal of Developmental and Behavioral Pediatrics : JDBP, 31(4), 346-356. http://doi.org/10.1097/DBP.0b013e3181dcaa8b

Fields, R. D. (2008). White matter in learning, cognition and psychiatric disorders. Trends in Neurosciences, 31(7), 361-70. http://doi.org/10.1016/j.tins.2008.04.001 
Filippi, M. (2001). Magnetic resonance imaging findings predicting subsequent disease course in patients at presentation with clinically isolated syndromes suggestive of multiple sclerosis. Neurological Sciences, 22(SUPPL. 2). http://doi.org/10.1007/s100720100033

Filippi, M., Iannucci, G., Cercignani, M., Assunta Rocca, M., Pratesi, A., \& Comi, G. (2000). A quantitative study of water diffusion in multiple sclerosis lesions and normal-appearing white matter using echo-planar imaging. Arch Neurol, 57(7), 1017-1021. http://doi.org/noc90075 [pii]

Filippi, M., Rossi, P., Campi, A., Colombo, B., Pereira, C., \& Comi, G. (1997). Serial contrastenhanced MR in patients with multiple sclerosis and varying levels of disability. American Journal of Neuroradiology, 18(8), 1549-1556.

Geurts, J. J., \& Barkhof, F. (2008). Grey matter pathology in multiple sclerosis. The Lancet Neurology, 7(9), 841-851. http://doi.org/10.1016/S1474-4422(08)70191-1

Godin, G. (2011). The Godin-Shephard Leisure-Time Physical Activity Questionnaire. Health \& Fitness Journal of Canada, 4(1), 18-22.

Gorman, M. P., Healy, B. C., Polgar-Turcsanyi, M., \& Chitnis, T. (2009a). Increased Relapse Rate in Pediatric-Onset Compared With Adult-Onset Multiple Sclerosis. Archives of Neurology, 66(1), 54-59. http://doi.org/10.1001/archneurol.2008.505

Gorman, M. P., Healy, B. C., Polgar-Turcsanyi, M., \& Chitnis, T. (2009b). Increased relapse rate in pediatric-onset compared with adult-onset multiple sclerosis. Archives of Neurology, 66(1), 54-59. http://doi.org/10.1001/archneurol.2008.505

Grassi-Oliveira, R., Stein, L. M., Lopes, R. P., Teixeira, A. L., \& Bauer, M. E. (2008). Low Plasma Brain-Derived Neurotrophic Factor and Childhood Physical Neglect Are Associated with Verbal Memory Impairment in Major Depression-A Preliminary Report. Biological Psychiatry, 64(4), 281-285. http://doi.org/10.1016/j.biopsych.2008.02.023

Griesbach, G. S. (2011). Exercise After Traumatic Brain Injury: Is it a Double-Edged Sword? PMRJ, 3(6), S64-S72. http://doi.org/10.1016/j.pmrj.2011.02.008

Grover, A., Aubert-Broche, B., Fetco, D., Collins Louis, D., Arnold, L., Finlayson, M., ... Yeh Ann, E. (2015). Lower physical activity is associated with higher disease burden in pediatric multiple sclerosis. Neurology, 85(19), 1663.

Gunnarsson, S. (2014). Non-Gaussian Diffusion Imaging : A comparison of the bi-Gaussian and the fourth-order tensor models to diffusion tensor imaging. 
Guo, A. C., Jewells, V. L., \& Provenzale, J. M. (2001). Analysis of normal-appearing white Matter in multiple sclerosis: Comparison of diffusion tensor MR imaging and magnetization transfer imaging. American Journal of Neuroradiology, 22(10), 1893-1900.

Hartline, D. K. (2008). What is myelin? Neuron Glia Biology, 4(2), 153-163. http://doi.org/10.1017/S1740925X09990263

Hosseini, B., Flora, D. B., Banwell, B. L., \& Till, C. (2014). Age of onset as a moderator of cognitive decline in pediatric-onset multiple sclerosis. Journal of the International Neuropsychological Society : JINS, 20(8), 796-804. http://doi.org/10.1017/S1355617714000642

Hulst, H. E., Steenwijk, M. D., Versteeg, A., Pouwels, P. J. W., Vrenkan, H., Uitdehaag, B. M. J., ... Barkhof, F. (2013). Cognitive impairment in MS: Imapct of white matter integrity, gray matter volume, and lesions. Neurology, 80, 1025-1032. http://doi.org/10.1212/WNL.0b013e31828726cc

Hurford, J. R. (1991). The evolution of the critical period for language acquisition. Cognition, 40(3), 159-201. http://doi.org/10.1016/0010-0277(91)90024-X

Ibrahim, I., Tintera, J., Skoch, A., Jirů, F., Hlustik, P., Martinkova, P., ... Rasova, K. (2011). Fractional anisotropy and mean diffusivity in the corpus callosum of patients with multiple sclerosis: The effect of physiotherapy. Neuroradiology, 53(11), 917-926. http://doi.org/10.1007/s00234-011-0879-6

Jacobs, D. R., Ainsworth, B. E., Hartman, T. J., \& Leon, A. S. (1993). A simultaneous evaluation of 10 commonly used physical activity questionnaires. Medicine and Science in Sports and Exercise, 25(1), 81-91. http://doi.org/10.1017/CBO9781107415324.004

Jerstad, S. J., Boutelle, K. N., Ness, K. K., \& Stice, E. (2010). Prospective reciprocal relations between physical activity and depression in female adolescents. Journal of Consulting and Clinical Psychology, 78(2), 268-72. http://doi.org/10.1037/a0018793

Kail, R. (2000). Speed of information processing: Developmental change and links to intelligence. Journal of School Psychology, 38(1), 51-61. http://doi.org/10.1016/S00224405(99)00036-9

Kail, R., \& Salthouse, T. a. (1994). Processing speed as a mental capacity. Acta Psychologica, 86(2-3), 199-225. http://doi.org/10.1016/0001-6918(94)90003-5

Kerbrat, A., Aubert-Broche, B., Fonov, V., Narayanan, S., Sled, J. G., Arnold, D. A., ... Collins, 
D. L. (2012). Reduced head and brain size for age and disproportionately smaller thalami in child-onset MS. Neurology, 78(3), 194-201.

http://doi.org/10.1212/WNL.0b013e318240799a

Klawiter, E. C., Schmidt, R. E., Trinkaus, K., Liang, H. F., Budde, M. D., Naismith, R. T., ...

Benzinger, T. L. (2011). Radial diffusivity predicts demyelination in ex vivo multiple sclerosis spinal cords. NeuroImage, 55(4), 1454-1460.

http://doi.org/10.1016/j.neuroimage.2011.01.007

Klingberg, T., Vaidya, C. J., Gabrieli, J. D., Moseley, M. E., \& Hedehus, M. (1999). Myelination and organization of the frontal white matter in children: a diffusion tensor MRI study. Neuroreport, 10(13), 2817-2821. http://doi.org/10.1097/00001756-199909090-00022

Koch-Henriksen, N., \& Sørensen, P. S. (2010). The changing demographic pattern of multiple sclerosis epidemiology. The Lancet Neurology, 9(5), 520-532.

http://doi.org/10.1016/S1474-4422(10)70064-8

Kochunov, P., Thompson, P. M., Lancaster, J. L., Bartzokis, G., Smith, S., Coyle, T., ... Fox, P. T. (2007). Relationship between white matter fractional anisotropy and other indices of cerebral health in normal aging: Tract-based spatial statistics study of aging. NeuroImage, 35(2), 478-487. http://doi.org/10.1016/j.neuroimage.2006.12.021

Kolasinski, J., Stagg, C. J., Chance, S. A., Deluca, G. C., Esiri, M. M., Chang, E.-H., ... Johansen-Berg, H. (2012). A combined post-mortem magnetic resonance imaging and quantitative histological study of multiple sclerosis pathology. Brain : A Journal of Neurology, 135(Pt 10), 2938-51. http://doi.org/10.1093/brain/aws242

Krogsrud, S. K., Fjell, A. M., Tamnes, C. K., Grydeland, H., Mork, L., Due-Tønnessen, P., ... Walhovd, K. B. (2016). Changes in white matter microstructure in the developing brain-A longitudinal diffusion tensor imaging study of children from 4 to 11 years of age. NeuroImage, 124, 473-486. http://doi.org/10.1016/j.neuroimage.2015.09.017

Kurtzke, J. F. (1983). Rating neurologic impairment in multiple sclerosis: an expanded disability status scale (EDSS). Neurology, 33(11), 1444-1452. http://doi.org/10.1212/WNL.33.11.1444

Lai, M., Hodgson, T., Gawne-Cain, M., Webb, S., MacManus, D., McDonald, W. I., ... Miller, D. H. (1996). A preliminary study into the sensitivity of disease activity detection by serial weekly magnetic resonance imaging in multiple sclerosis. J.Neurol.Neurosurg.Psychiatry, 
60(3), 339-341.

Lakes, K. D., \& Hoyt, W. T. (2004). Promoting self-regulation through school-based martial arts training. Journal of Applied Developmental Psychology, 25(3), 283-302. http://doi.org/10.1016/j.appdev.2004.04.002

Larsson, H. B., Thomsen, C., Frederiksen, J., Stubgaard, M., \& Henriksen, O. (1992). In vivo magnetic resonance diffusion measurement in the brain of patients with multiple sclerosis. Magnetic Resonance Imaging, 10(1), 7-12.

Laule, C., Kozlowski, P., Leung, E., Li, D. K. B., MacKay, A. L., \& Moore, G. R. W. (2008). Myelin water imaging of multiple sclerosis at 7??T: Correlations with histopathology. NeuroImage, 40(4), 1575-1580. http://doi.org/10.1016/j.neuroimage.2007.12.008

Lebel, C., \& Beaulieu, C. (2011). Longitudinal development of human brain wiring continues from childhood into adulthood. The Journal of Neuroscience, 31(30), 10937-10947. http://doi.org/10.1523/JNEUROSCI.5302-10.2011

Leow, A. D., Zhan, L., Zhu, S., Hageman, N., Chiang, M. C., Barysheva, M., .. Thompson, P. M. (2009). White matter integrity measured by fractional anisotropy correlates poorly with actual individual fiber anisotropy. Proceedings - 2009 IEEE International Symposium on Biomedical Imaging: From Nano to Macro, ISBI 2009, (March 2017), 622-625. http://doi.org/10.1109/ISBI.2009.5193124

Levin, H. S., Mendelsohn, D., Lilly, M. A., Yeakley, J., Song, J., Scheibel, R. S., .. Bruce, D. (1997). Magnetic resonance imaging in relation to functional outcome of pediatric closed head injury: A test of the Ommaya-Gennarelli model. Neurosurgery, 40(3), 432-441. http://doi.org/10.1097/00006123-199703000-00002

Longoni, G., Brown, R. A., Momayyezsiahkal, P., Elliott, C., Narayanan, S., Bar-Or, A., ... Arnold, D. L. (2017). White matter changes in paediatric multiple sclerosis and monophasic demyelinating disorders. Brain, 1-16. http://doi.org/10.1093/brain/awx041

Lublin, F. D., Reingold, S. C., Cohen, J. a, Cutter, G. R., Thompson, A. J., Wolinsky, J. S., ... Lubetzki, C. (2014). Defining the clinical course of multiple sclerosis : The 2013 revisions Defining the clinical course of multiple sclerosis The 2013 revisions, $1-10$. http://doi.org/10.1212/WNL.0000000000000560

MacAllister, W. S., Boyd, J. R., Holland, N. J., Milazzo, M. C., \& Krupp, L. B. (2007). The psychosocial consequences of pediatric multiple sclerosis. Neurology, 68(16 SUPPL. 2). 
http://doi.org/10.1212/01.wnl.0000259420.54635.63

MacAllister, W. S., Christodoulou, C., Milazzo, M., \& Krupp, L. B. (2007). Longitudinal neuropsychological assessment in pediatric multiple sclerosis. Developmental Neuropsychology, 32(2), 625-644. http://doi.org/10.1080/87565640701375872

MacAllister, W. S., Christodoulou, C., Troxell, R., Milazzo, M., Block, P., Preston, T. E., ... Krupp, L. B. (2009). Fatigue and quality of life in pediatric multiple sclerosis. Multiple Sclerosis (Houndmills, Basingstoke, England), 15(12), 1502-1508. http://doi.org/10.1177/1352458509345902

MacAllister WS Milazzo M, Weisbrot DM, Christodoulou C, Scherl WF, Preston TE, Cianciulli C, Krupp LB., B. A. L. (2005). Cognitive functioning in children and adolescents with multiple sclerosis. Neurology, 64(8), 1422-1425. http://doi.org/10.1212/01.WNL.0000158474.24191.BC

Mackay, A. L., \& Laule, C. (2012). Chapter 28 Myelin Water Imaging. In G. M. Bydder, G. D. Fullerton, \& I. R. Young (Eds.), MRI of Tissues with Short T2s or T2*s (pp. 359-376). John Wiley \& Sons, Ltd.

Manjunath, N. K., \& Telles, S. (2001). Improved performance in the Tower of London test following yoga. Indian Journal of Physiology and Pharmacology, 45(3), 351-354.

Marrie, R. A. (2004). Environmental risk factors in multiple sclerosis aetiology. The Lancet Neurology, 3(12), 709-718. http://doi.org/10.1016/S1474-4422(04)00933-0

Mathiowetz, V., Weber, K., Kashman, N., \& Volland, G. (1985). Adult norms for the Nine Hole Peg Test of finger dexterity. The Occupational Therapy Journal of Research, 5(1), 24-38. http://doi.org/10.1177/153944928500500102

Mesaros, S., Rocca, M.A., Kacar, K., Kostic, J., Copetti, M., Stosic-Opincal, T., Preziosa, P., Sala, AS., Riccitelli, G., Horsfield, M.A., Drulovic, J., Comi, G., Filippi, M. (2012). Diffusion tensor MRI tractography and cognitive impairment in multiple sclerosis, 969975.

Mesaros, S., Rocca, M. A., Absinta, M., Ghezzi, A., Milani, N., Moiola, L., ... Filippi, M. (2008). Evidence of thalamic gray matter loss in pediatric multiple sclerosis. Neurology, 70(13 PART 2), 1107-1112. http://doi.org/10.1212/01.wnl.0000291010.54692.85

Miles, L., Grossman, R. I., Johnson, G., Babb, J. S., Diller, L., \& Inglese, M. (2008). Short-term DTI predictors of cognitive dysfunction in mild traumatic brain injury. Brain Injury : [BI], 
22(2), 115-122. http://doi.org/10.1080/02699050801888816

Milo, R., \& Kahana, E. (2010). Autoimmunity Reviews Multiple sclerosis : Geoepidemiology, genetics and the environment. Autoimmunity Reviews, 9(5), A387-A394. http://doi.org/10.1016/j.autrev.2009.11.010

Mori, S., \& Zhang, J. (2006). Principles of Diffusion Tensor Imaging and Its Applications to Basic Neuroscience Research. Neuron, 51(5), 527-539. http://doi.org/10.1016/j.neuron.2006.08.012

Motl, R. W., \& Sandroff, B. M. (2015). Benefits of Exercise Training in Multiple Sclerosis. Current Neurology and Neuroscience Reports, 15(9), 62. http://doi.org/10.1007/s11910015-0585-6

Neeper, S., Gomez-Pinilla, F., Choi, J., \& Cotman, C. (1995). Exercise and Brain Neurotrophins. Nature, 373, 109.

Paus, T., Zijdenbos, A., Worsley, K., Collins, D. L., Blumenthal, J., Giedd, J. N., ... Evans, A. C. (1999). Structural maturation of neural pathways in children and adolescents: in vivo study. Science (New York, N.Y.), 283(5409), 1908-1911. http://doi.org/10.1126/science.283.5409.1908

Pereira, A. C., Huddleston, D. E., Brickman, A. M., Sosunov, A. A., Hen, R., McKhann, G. M., ... Brown, T. R. (2007). An in vivo correlate of exercise-induced neurogenesis in the adult dentate gyrus. Proceedings of the National Academy of Sciences of the United States of America, 104(13), 5638-43. http://doi.org/10.1073/pnas.0611721104

Polman, C. H., Reingold, S. C., Banwell, B., Clanet, M., Cohen, J. A., Filippi, M., ... Connor, P. O. (2011). Diagnostic Criteria for Multiple Sclerosis : 2010 Revisions to the McDonald Criteria. Annals of Neurology, 69(2), 292-302. http://doi.org/10.1002/ana.22366

Portaccio, E., Goretti, B., Lori, S., Zipoli, V., Centorrino, S., Ghezzi, A., ... Amato, M. P. (2009). The brief neuropsychological battery for children: a screening tool for cognitive impairment in childhood and juvenile multiple sclerosis. Multiple Sclerosis (Houndmills, Basingstoke, England), 15(November 2008), 620-626. http://doi.org/10.1177/1352458508101950

Portaccio, E., Goretti, B., Zipoli, V., Hakiki, B., Giannini, M., Pastò, L., ... Amato, M. P. (2010). Cognitive rehabilitation in children and adolescents with multiple sclerosis. Neurological Sciences : Official Journal of the Italian Neurological Society and of the Italian Society of 
Clinical Neurophysiology, 31(Suppl 2), S275-8. http://doi.org/10.1007/s10072-010-0377-3

Prakash, R. S., Patterson, B., Janssen, A., Abduljalil, A., \& Boster, A. (2011). Physical Activity Associated with Increased Resting-State Functional Connectivity in Multiple Sclerosis. Journal of the International Neuropsychological Society, 17, 986-997. http://doi.org/10.1017/S1355617711001093

Prakash, R. S., Snook, E. M., Motl, R. W., \& Kramer, A. F. (2010). Aerobic fitness is associated with gray matter volume and white matter integrity in multiple sclerosis. Brain Research, 1341, 41-51. http://doi.org/10.1016/j.brainres.2009.06.063

Prosperini, L., Fanelli, F., Petsas, N., Sbardella, E., Tona, F., Raz, E., \& Pantano, P. (2014). Multiple Sclerosis: Changes in Microarchitecture of White Matter Tracts after Training Title. Radiology, 273(2), 529-538.

Prosperini, L., Piattella, M. C., Giannì, C., \& Pantano, P. (2015). Functional and Structural Brain Plasticity Enhanced by Motor and Cognitive Rehabilitation in Multiple Sclerosis. Neural Plasticity, 2015, 481574. http://doi.org/10.1155/2015/481574

Rasova, K., Prochazkova, M., Tintera, J., Ibrahim, I., Zimova, D., \& Stetkarova, I. (2015). Motor programme activating therapy influences adaptive brain functions in multiple sclerosis. International Journal of Rehabilitation Research, 38(1), 49-54. http://doi.org/10.1097/MRR.0000000000000090

Raz, E., Cercignani, M., Sbardella, E., Totaro, P., Pozzilli, C., Bozzali, M., \& Pantano, P. (2010). Clinically isolated syndrome suggestive of multiple sclerosis: voxelwise regional investigation of white and gray matter. Radiology, 254(1), 227-234. http://doi.org/10.1148/radiol.2541090817

Renoux, C., Vukusic, S., Mikaeloff, Y., Edan, G., Clanet, M., Dubois, B., ... Confavreux, C. (2007). Natural history of multiple sclerosis with childhood onset. The New England Journal of Medicine, 356(25), 2603-2613. http://doi.org/10.1056/NEJMoa067597

Rocca, M. A., Cercignani, M., Iannucci, G., Comi, G., \& Filippi, M. (2000). Weekly diffusionweighted imaging of normal-appearing white matter in MS. Neurology. http://doi.org/10.1212/WNL.55.6.882

Rocca, M. a., Valsasina, P., Absinta, M., Moiola, L., Ghezzi, A., Veggiotti, P., ... Filippi, M. (2014). Intranetwork and internetwork functional connectivity abnormalities in pediatric multiple sclerosis. Human Brain Mapping, 35(8), 4180-4192. 
http://doi.org/10.1002/hbm.22469

Rocca, M. a, Absinta, M., Amato, M. P., Moiola, L., Ghezzi, A., Veggiotti, P., ... Filippi, M. (2014). Posterior brain damage and cognitive impairment in pediatric multiple sclerosis. Neurology, 82(15), 1314-21. http://doi.org/10.1212/WNL.0000000000000309

Rocca, M. a, Amato, M. P., De Stefano, N., Enzinger, C., Geurts, J. J., Penner, I.-K., ... Filippi, M. (2015). Clinical and imaging assessment of cognitive dysfunction in multiple sclerosis. The Lancet. Neurology, 14(3), 302-317. http://doi.org/10.1016/S1474-4422(14)70250-9

Rocca, M., De Meo, E., Amato, M., Copetti, M., Moiola, L., Ghezzi, A., ... Filippi, M. (2015). Cognitive impairment in paediatric multiple sclerosis patients is not related to cortical lesions. Multiple Sclerosis (Houndmills, Basingstoke, England), 21(7), 956-959. http://doi.org/10.1177/1352458514557303

Roosendaal, S. D., Geurts, J. J. G., Vrenken, H., Hulst, H. E., Cover, K. S., Castelijns, J. A., .. Barkhof, F. (2009). Regional DTI differences in multiple sclerosis patients. NeuroImage, 44(4), 1397-1403. http://doi.org/10.1016/j.neuroimage.2008.10.026

Rovaris, M., Iannucci, G., Falautano, M., Possa, F., Martinelli, V., Comi, G., \& Filippi, M. (2002). Cognitive dysfunction in patients with mildly disabling relapsing-remitting multiple sclerosis: an exploratory study with diffusion tensor MR imaging. J Neurol Sci, 195(2), 103-109. http://doi.org/S0022510X01006906 [pii]

Rypma, B., Berger, J. S., Prabhakaran, V., Martin Bly, B., Kimberg, D. Y., Biswal, B. B., \& D’Esposito, M. (2006). Neural correlates of cognitive efficiency. NeuroImage, 33, 969-979. http://doi.org/10.1016/j.neuroimage.2006.05.065

Sallis, J. F., Buono, M. J., Roby, J. J., Micale, F. G., \& Nelson, J. A. (1993). Seven-day recall and other physical activity self-reports in children and adolescents. Medicine and Science in Sports and Exercise, 25(1), 99-108. http://doi.org/10.1249/00005768-199301000-00014

Sbardella, E., Tona, F., Petsas, N., \& Pantano, P. (2013). Review Article. DTI Measurements in Multiple Sclerosis : Evaluation of Brain Damage and Clinical Implications. Multiple Sclerosis International, 2013, 11.

Scanderbeg, A. C., Tomaiuolo, F., Sabatini, U., Nocentini, U., Grasso, M. G., \& Caltagirone, C. (2000). Demyelinating plaques in relapsing-remitting and secondary-progressive multiple sclerosis: Assessment with diffusion MR imaging. American Journal of Neuroradiology, $21(5), 862-868$. 
Schmierer, K., Wheeler-Kingshott, C. A. M., Boulby, P. A., Scaravilli, F., Altmann, D. R., Barker, G. J., .. Miller, D. H. (2007). Diffusion tensor imaging of post mortem multiple sclerosis brain. NeuroImage, 35(2), 467-477. http://doi.org/10.1016/j.neuroimage.2006.12.010

Schmithorst, V. J., \& Yuan, W. (2010). White matter development during adolescence as shown by diffusion MRI. Brain and Cognition, 72(1), 16-25. http://doi.org/10.1016/j.bandc.2009.06.005

Schoonheim, M. M., Meijer, K. A., \& Geurts, J. J. G. (2015). Network collapse and cognitive impairment in multiple sclerosis. Frontiers in Neurology, 6(82), 1-5. http://doi.org/10.3389/fneur.2015.00082

Schrank, F. A. (2010). Woodcock-Johnson III Tests of Cognitive Abilities. Handbook of Pediatric Neuropsychology, 371-401.

Schrank, F. A., \& Woodcock, R. W. (2001). Woodcock-Johnson III, (2).

Singleton, D. (2005). The Critical Period Hypothesis : A coat of many colours. IRAL, 43, 269285.

Smith, A. (1968). The Symbol-Digit Modalities Test: A neuropsychologic test for economic screening of learning and other cerebral disorders. Learning Disorders, 3, 83-91.

Smith, S. M., Jenkinson, M., Johansen-Berg, H., Rueckert, D., Nichols, T. E., Mackay, C. E., ... Behrens, T. E. J. (2006). Tract-based spatial statistics: Voxelwise analysis of multi-subject diffusion data. NeuroImage, 31(4), 1487-1505. http://doi.org/10.1016/j.neuroimage.2006.02.024

Smith, S. M., Smith, S. M., Johansen-berg, H., Johansen-berg, H., Jenkinson, M., Jenkinson, M., ... Behrens, T. E. J. (2007). Acquisition and voxelwise analysis of multi-subject diffusion data with tract-based spatial statistics. Nature Protocols, 2(3), 499-503. http://doi.org/10.1038/nprot.2007.45

Smith, S. M., Zhang, Y., Jenkinson, M., Chen, J., Matthews, P. M., Federico, A., \& De Stefano, N. (2002). Accurate, robust, and automated longitudinal and cross-sectional brain change analysis. NeuroImage, 17(1), 479-89. http://doi.org/10.1006/nimg.2002.1040

Song, S.-K., Sun, S.-W., Ramsbottom, M. J., Chang, C., Russell, J., \& Cross, A. H. (2002). Dysmyelination Revealed through MRI as Increased Radial (but Unchanged Axial) Diffusion of Water. NeuroImage, 17(3), 1429-1436. http://doi.org/10.1006/nimg.2002.1267 
Strien, J. W. Van. (1992). Classificatie van links- en rechtshandige proefpersonen (Classification of left- and right-handed subjects.). Nederlands Tijdschrift Voor de Psychologie En Haar Grensgebieden, 47, 88-92.

Szuhany, K. L., Bugatti, M., \& Otto, M. W. (2015). A meta-analytic review of the effects of exercise on brain-derived neurotrophic factor. Journal of Psychiatric Research, 60, 56-64. http://doi.org/10.1016/j.jpsychires.2014.10.003

Tamnes, C. K., Ostby, Y., Fjell, A. M., Westlye, L. T., Due-Tønnessen, P., \& Walhovd, K. B. (2010). Brain maturation in adolescence and young adulthood: regional age-related changes in cortical thickness and white matter volume and microstructure. Cereb Cortex, 20(3), 534-548. http://doi.org/10.1093/cercor/bhp118

Taubert, M., Draganski, B., Anwander, A., Muller, K., Horstmann, A., Villringer, A., \& Ragert, P. (2010). Dynamic properties of human brain structure: learning-related changes in cortical areas and associated fiber connections 3. J Neurosci., 30(1529-2401 (Electronic)), 1167011677. http://doi.org/10.1523/JNEUROSCI.2567-10.2010

Thompson, A. J., Kermode, A. G., MacManus, D. G., Kendall, B. E., Kingsley, D. P., Moseley, I. F., \& McDonald, W. I. (1990). Patterns of disease activity in multiple sclerosis: clinical and magnetic resonance imaging study. BMJ, 300(6725), 631-634.

http://doi.org/10.1136/bmj.300.6725.631

Till, C., Deotto, A., Tipu, V., Sled, J., Bethune, A., Narayanan, S., .. Banwell, B. (2011). White matter integrity and math performance in pediatric multiple sclerosis: a diffusion tensor imaging study. Neuroreport, 22(18), 1005-1009. http://doi.org/10.1097/WNR.0b013e32834dc301

Till, C., Ghassemi, R., Aubert-Broche, B., Kerbrat, a, Collins, D. L., Narayanan, S., ... Banwell, B. L. (2011). MRI correlates of cognitive impairment in childhood-onset multiple sclerosis. Neuropsychology, 25(3), 319-332. http://doi.org/10.1037/a0022051

Till, C., Ho, C., Dudani, a, Garcia-Lorenzo, D., Collins, D. L., \& Banwell, B. L. (2012). Magnetic resonance imaging predictors of executive functioning in patients with pediatriconset multiple sclerosis. Arch Clin Neuropsychol, 27(5), 495-509. http://doi.org/acs058 [pii] \n10.1093/arclin/acs058

Till, C., Racine, N., Araujo, D., Narayanan, S., Collins, D. L., Aubert-Broche, B., ... Banwell, B. (2013a). Changes in cognitive performance over a 1-year period in children and adolescents 
with multiple sclerosis. Neuropsychology, 27(2), 210-9. http://doi.org/10.1037/a0031665

Till, C., Racine, N., Araujo, D., Narayanan, S., Collins, D. L., Aubert-Broche, B., ... Banwell, B. (2013b). Changes in cognitive performance over a 1-year period in children and adolescents with multiple sclerosis. Neuropsychology, 27(2), 210-9. http://doi.org/10.1037/a0031665

Tillema, J. M., Leach, J., \& Pirko, I. (2012). Non-lesional white matter changes in pediatric multiple sclerosis and monophasic demyelinating disorders. Multiple Sclerosis (Houndmills, Basingstoke, England), 18(12), 1754-9. http://doi.org/10.1177/1352458512447527

Todorow, M., DeSouza, J. F., Banwell, B. L., \& Till, C. (2014). Interhemispheric cooperation in global-local visual processing in pediatric multiple sclerosis. Journal of Clinical and Experimental Neuropsychology, 36(0), 111-26.

http://doi.org/10.1080/13803395.2013.867013

Tortorella, P., Rocca, M. A., Mezzapesa, D. M., Ghezzi, A., Lamantia, L., Comi, G., \& Filippi, M. (2006). MRI quantification of gray and white matter damage in patients with early-onset multiple sclerosis. Journal of Neurology, 253(7), 903-907. http://doi.org/10.1007/s00415006-0129-8

Tsang, J. M., Dougherty, R. F., Deutsch, G. K., Wandell, B. a, \& Ben-Shachar, M. (2009). Frontoparietal white matter diffusion properties predict mental arithmetic skills in children. Proceedings of the National Academy of Sciences of the United States of America, 106(52), 22546-22551. http://doi.org/10.1073/pnas.0906094106

van Eimeren, L., Grabner, R. H., Koschutnig, K., Reishofer, G., Ebner, F., \& Ansari, D. (2010). Structure-function relationships underlying calculation: A combined diffusion tensor imaging and fMRI study. NeuroImage, 52(1), 358-363. http://doi.org/10.1016/j.neuroimage.2010.04.001

Van Hecke, W., Nagels, G., Leemans, A., Vandervliet, E., Sijbers, J., \& Parizel, P. M. (2010). Correlation of cognitive dysfunction and diffusion tensor MRI measures in patients with mild and moderate multiple sclerosis. Journal of Magnetic Resonance Imaging, 31(6), 1492-1498. http://doi.org/10.1002/jmri.22198

Van Waesberghe, J. H. T. M., Kamphorst, W., De Groot, C. J. A., Van Walderveen, M. A. A., Castelijns, J. A., Ravid, R., ... Barkhof, F. (1999). Axonal loss in multiple sclerosis lesions: Magnetic resonance imaging insights into substrates of disability. Annals of Neurology, 46(5), 747-754. http://doi.org/10.1002/1531-8249(199911)46:5<747::AID- 


\section{ANA10>3.0.CO;2-4}

Varni, J. W., Seid, M., \& Rode, C. a. (1999). The PedsQL: measurement model for the pediatric quality of life inventory. Medical Care, 37(2), 126-39. http://doi.org/10.1186/1477-7525$11-47$

Verburgh, L., Konigs, M., Scherder, E. J. A., \& Oosterlaan, J. (2013). Physical exercise and executive functions in preadolescent children, adolescents and young adults: a metaanalysis. British Journal of Sports Medicine, 48(12), 973-979.

http://doi.org/10.1136/bjsports-2012-091441

Verger, K., Junqué, C., Levin, H. S., Jurado, M. a, Pérez-Gómez, M., Bartrés-Faz, D., ... Mercader, J. M. (2001). Correlation of atrophy measures on MRI with neuropsychological sequelae in children and adolescents with traumatic brain injury. Brain Injury: [BI], 15(3), 211-221. http://doi.org/10.1080/02699050010004059

Verhey, L. H., Branson, H. M., Shroff, M. M., Callen, D. J. A., Sled, J. G., Narayanan, S., ... Bar-or, A. (2011). MRI parameters for prediction of multiple sclerosis diagnosis in children with acute CNS demyelination : a prospective national cohort study. The Lancet Neurology, 10(12), 1065-1073. http://doi.org/10.1016/S1474-4422(11)70250-2

Vishwas, M. S., Chitnis, T., Pienaar, R., Healy, B. C., \& Grant, P. E. (2010a). Tract-based analysis of callosal, projection, and association pathways in pediatric patients with multiple sclerosis: A preliminary study. American Journal of Neuroradiology, 31(1), 121-128. http://doi.org/10.3174/ajnr.A1776

Vishwas, M. S., Chitnis, T., Pienaar, R., Healy, B. C., \& Grant, P. E. (2010b). Tract-Based Analysis of Callosal, Projection, and Association Pathways in Pediatric Patients with Multiple Sclerosis: A Preliminary Study. American Journal of Neuroradiology, 31(1), 121128. http://doi.org/10.3174/ajnr.A1776

Vishwas, M. S., Healy, B. C., Pienaar, R., Gorman, M. P., Grant, P. E., \& Chitnis, T. (2013). Diffusion tensor analysis of pediatric multiple sclerosis and clinically isolated syndromes. AJNR. American Journal of Neuroradiology, 34(2), 417-23. http://doi.org/10.3174/ajnr.A3216

Vos, S. B., Jones, D. K., Jeurissen, B., Viergever, M. A., \& Leemans, A. (2012). The influence of complex white matter architecture on the mean diffusivity in diffusion tensor MRI of the human brain. NeuroImage, 59(3), 2208-2216. 
http://doi.org/10.1016/j.neuroimage.2011.09.086

Voss, M. W., Vivar, C., Kramer, A. F., \& van Praag, H. (2013). Bridging animal and human models of exercise-induced brain plasticity. Trends in Cognitive Sciences, 17(10), 525-544. http://doi.org/10.1016/j.tics.2013.08.001

Wang, Z., Sadovnick, A. D., Traboulsee, A. L., Zhang, S., Wang, Z., Sadovnick, A. D., ... Encarnacion, M. (2016). Nuclear Receptor NR1H3 in Familial Multiple Report Nuclear Receptor NR1H3 in Familial Multiple Sclerosis, 948-954. http://doi.org/10.1016/j.neuron.2016.04.039

Wechsler, D. (2013). WASI -II: Wechsler abbreviated scale of intelligence - second edition. Journal of Psychoeducational Assessment, 31(3), 337-41. http://doi.org/10.1177/0734282912467756

Werring, D. J., Clark, C. A., Barker, G. J., Thompson, A. J., \& Miller, D. H. (1999). Diffusion tensor imaging of lesions and normal-appearing white matter in multiple sclerosis. Neurology, 52(8), 1626-1626. http://doi.org/10.1212/WNL.52.8.1626

Wozniak, J. R., Krach, L., Ward, E., Mueller, B. A., Muetzel, R., Schnoebelen, S., ... Lim, K. O. (2007). Neurocognitive and neuroimaging correlates of pediatric traumatic brain injury: A diffusion tensor imaging (DTI) study. Archives of Clinical Neuropsychology, 22(5), 555568. http://doi.org/10.1016/j.acn.2007.03.004

Yeh, E. A., Chitnis, T., Krupp, L., Ness, J., Chabas, D., Kuntz, N., \& Waubant, E. (2009). Pediatric multiple sclerosis. Nature Reviews Neurology, 5(11), 621-631. http://doi.org/10.1038/nrneurol.2009.158

Yeh, E. A., Kinnett-Hopkins, D., Grover, S. A., \& Motl, R. W. (2015). Physical activity and pediatric multiple sclerosis: Developing a research agenda. Multiple Sclerosis, 21(13), 1618-1625. http://doi.org/10.1177/1352458515606526

Yeh, E. A., Weinstock-Guttman, B., Ramanathan, M., Ramasamy, D. P., Willis, L., Cox, J. L., \& Zivadinov, R. (2009). Magnetic resonance imaging characteristics of children and adults with paediatric-onset multiple sclerosis. Brain, 132(12), 3392-3400. http://doi.org/10.1093/brain/awp278

Yu, H. J., Christodoulou, C., Bhise, V., Greenblatt, D., Patel, Y., Serafin, D., ... Wagshul, M. E. (2012). Multiple white matter tract abnormalities underlie cognitive impairment in RRMS. NeuroImage, 59(4), 3713-3722. http://doi.org/10.1016/j.neuroimage.2011.10.053 
Zahr, N., Rohlfing, T., Pfefferbaum, A., \& Sullivan, E. (2008). Quantitative fiber tracking in the normal aging brain: neuropsychological correlates. In Proceedings 16th Scientific Meeting, International Society for Magnetic Resonance in Medicine (Vol. Toronto, p. 2098).

Zhang, H., Schneider, T., Wheeler-Kingshott, C. A., \& Alexander, D. C. (2012). NODDI: Practical in vivo neurite orientation dispersion and density imaging of the human brain. NeuroImage, 61(4), 1000-1016. http://doi.org/10.1016/j.neuroimage.2012.03.072

Zivadinov, R., \& Bakshi, R. (2004). Central nervous system atrophy and clinical status in multiple sclerosis. Journal of Neuroimaging : Official Journal of the American Society of Neuroimaging, 14(3 Suppl), 27S-35S. http://doi.org/10.1177/1051228404266266 


\subsection{APPENDIX A: CASE HISTORY FORM}

CASE HISTORY FORM

\section{This information is confidential and for professional use only.}

Date of MRI and cognitive testing: Time:

Subject ID number:

Name:

Date of Birth: (mm/dd/yy)

Current age:

Sex: $F$

Current address:

Current home phone number:

Alternative phone number:

Name of current/past school:

Current or highest grade level completed:

Mother's age:__ Mother's occupation:

Highest level of school completed by mother:

Father's age: ___ Father's occupation:

Highest level of school completed by father:

Current marital status of parents:

[] Married/Common-law [] Divorced [] Separated [] Widowed

City and Country of Birth:

Duration living in birth country:

If not from Canada, has your family been in Canada 5 years or more? [] yes [] no

First language spoken:

Most common language spoken at home: [] English [] Other:

[] Combination of English and other language

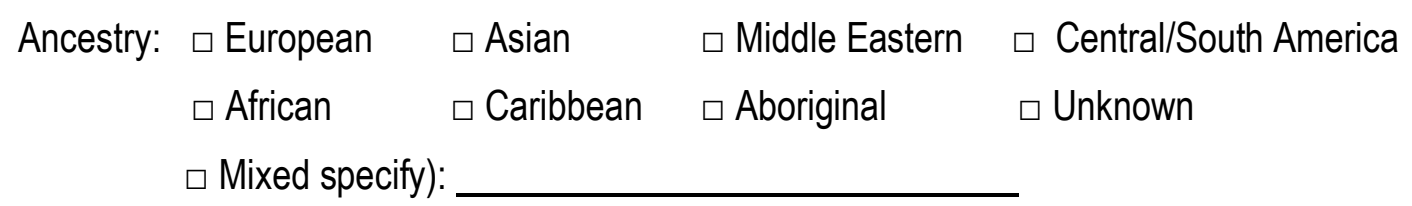


Race: $\square$ Caucasion $\square$ Oriental $\square$ Black $\square$ Unknown $\square$ Mixed:

\section{Medical History}

If you have MS, when was your last MS related attack?

Have you received any corticosteroid / IVlg treatment for an attack at least 6 weeks prior to participating in this research study? [] Yes [] No

Prescribed Medication at time of testing (including disease-modifying treatment, birth control) *used for more than 14 days within 3 months prior to study visit

\begin{tabular}{|l|l|l|l|l|}
\hline List & Dose & Ongoing $(\mathrm{Y} / \mathrm{N})$ & Duration & Reason for use \\
\hline & & & & \\
\hline & & & & \\
\hline & & & & \\
\hline
\end{tabular}

Supplements / Vitamins (including allergy, herbal or alternative meds)

*used for more than 14 days within 3 months prior to study visit

\begin{tabular}{|l|l|l|l|l|}
\hline List & Dose & Ongoing $(\mathrm{Y} / \mathrm{N})$ & Duration & Reason for use \\
\hline & & & & \\
\hline & & & & \\
\hline & & & & \\
\hline
\end{tabular}

Were you born full term? (>37 weeks) [] Yes [] No

Have you ever had a head injury? [ ] Yes [] No If yes, at what age?

Did you lose consciousness? [] Yes [] No For how long?

Do you wear [] Glasses? [] Contact lenses? Date of last vision test:

Reason for corrective lenses? (e.g. for distance, for reading):

Do you drink alcohol? [ ] No [ ] Yes

If yes how many drinks per week?

Do you use any illicit drugs (e.g. cannabis)? [ ] No [ ] Yes

If yes, which ones? and how often? 
Have you ever had or been diagnosed with the following:

Kidney disease

Epilepsy/seizures

Febrile convulsion

Respiratory disease

Heart disease

Hematological problems

Thyroid disease

Digestive problems

Diabetes

Hypertension

Circulatory disease

Cancer

Other
[] no

[] no

[] no

[] no

[] no

[] no

[] no

[] no

[] no

[] no

[] no

[] no

[] no
[ ] yes - explain:

[ ] yes - explain:

[] yes - explain:

[ ] yes - explain:

[ ] yes - explain:

[ ] yes - explain:

[ ] yes - explain:

[ ] yes - explain:

[ ] yes - explain:

[ ] yes - explain:

[ ] yes - explain:

[ ] yes - explain:

[ ] yes - explain:

\section{Behavioural and Mental Health History}

Have you ever been assessed by a psychologist or psychiatrist? [] No [] Yes

Reason and date:

Have you repeated a grade? [] No [] Yes

Which grade(s)?

Are you currently placed in a special education program? [] No [] Yes

Have you been placed in a special education program in the past? [] No [] Yes

Regular classroom? [] Yes [] No Modified classroom: [] Yes [] No

\section{Have you ever had or been diagnosed with the following:}

\begin{tabular}{|c|c|c|}
\hline Attention Deficit Disorder & [] no & [ ] yes - when: \\
\hline Attention Deficit/Hyperactivity Disorder & [] no & [ ] yes - when: \\
\hline Anxiety & [] no & [ ] yes - when: \\
\hline Autism Spectrum Disorder & [] no & [ ] yes - wher \\
\hline Depression & [] no & [] yes - when: \\
\hline Any psychiatric disorder & [] no & [] yes - wh \\
\hline
\end{tabular}


Learning Disability (LD)

[] no

[ ] yes - when:

Language Disorder

[] no

[] yes - when:

Giftedness

[] no

[ ] yes - when:

Please provide any additional comments you think might be helpful. 


\subsection{APPENDIX B. Supplementary Tables}

Supplementary Table 1. Correlations ${ }^{a}$ of average diffusion parameters (i.e., entire white matter skeleton) and disease characteristics in MS patients $(\mathrm{N}=23)$

\begin{tabular}{|l|l|l|l|}
\hline Clinical Characteristic & Fractional Anisotropy & Axial Diffusivity & Radial Diffusivity $^{\text {b }}$ \\
\hline Disease Duration & 0.114 & $-0.448^{*}$ & -0.262 \\
\hline Age of Onset & -0.116 & $0.444^{*}$ & 0.270 \\
\hline Number of relapses & -0.075 & -0.253 & -0.016 \\
\hline EDSS $^{c}$ Score & $-0.419^{*}$ & $0.423^{*}$ & $0.443^{*}$ \\
\hline Fatigue $^{\mathrm{d}}$ & 0.005 & 0.080 & -0.016 \\
\hline
\end{tabular}

$* p \leq 0.1$ (only trends shown)

${ }^{a}$ r-value reported

${ }^{b}$ Log-transformed

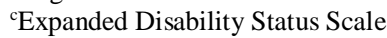

${ }^{\mathrm{d}}$ Based on the Pediatric Quality of Life Inventory Multidimensional Fatigue Scale 
Supplementary Table 2. Correlations ${ }^{\mathrm{a}}$ of diffusion parameters and select cognitive measures in MS

\begin{tabular}{|c|c|c|c|}
\hline & $\begin{array}{l}\text { SDMT } \\
\text { (z-score) }\end{array}$ & $\begin{array}{l}\text { WJ-III } \\
\text { Decision } \\
\text { Speed } \\
\text { (z-score) }\end{array}$ & $\begin{array}{l}\text { Dominant } \\
\text { Hand 9- } \\
\text { HPT (z- } \\
\text { score) }\end{array}$ \\
\hline \multicolumn{4}{|l|}{ Fractional Anisotropy } \\
\hline Entire white matter skeleton & 0.098 & 0.264 & 0.267 \\
\hline Corpus Callosum - Genu ${ }^{\mathrm{b}}$ & $0.414 *$ & 0.260 & 0.165 \\
\hline Corpus Callosum - Body & 0.127 & 0.381 & 0.193 \\
\hline Corpus Callosum - Splenium & -0.055 & 0.172 & 0.269 \\
\hline Cerebral Peduncle - Right ${ }^{\mathrm{b}}$ & -0.085 & 0.074 & 0.216 \\
\hline Cerebral Peduncle - Left ${ }^{\mathrm{b}}$ & -0.326 & -0.093 & -0.145 \\
\hline Internal Capsule - Right Anterior Limb & -0.074 & 0.259 & 0.155 \\
\hline Internal Capsule - Right Posterior Limb & $-0.367 *$ & -0.271 & -0.116 \\
\hline Internal Capsule - Left Posterior Limb & -0.176 & -0.163 & -0.186 \\
\hline Internal Capsule - Right Retrolenticular Part & 0.017 & 0.057 & -0.037 \\
\hline Internal Capsule - Left Retrolenticular Part & -0.089 & -0.126 & 0.015 \\
\hline Corona Radiata - Right Anterior & 0.199 & 0.127 & 0.170 \\
\hline Corona Radiata - Left Anterior & 0.246 & 0.345 & 0.324 \\
\hline Corona Radiata - Right Superior & -0.102 & -0.049 & 0.072 \\
\hline Corona Radiata - Left Superior & -0.063 & 0.028 & 0.071 \\
\hline Corona Radiata - Right Posterior & -0.110 & 0.104 & 0.053 \\
\hline Corona Radiata - Left Posterior & -0.007 & 0.061 & 0.003 \\
\hline Posterior Thalamic Radiation - Right $^{\mathrm{b}}$ & 0.086 & 0.256 & $0.378 *$ \\
\hline Posterior Thalamic Radiation - Left ${ }^{\mathrm{b}}$ & 0.113 & 0.197 & 0.344 \\
\hline Saggital Stratum - Right & -0.104 & 0.045 & -0.063 \\
\hline Saggital Stratum - Left & 0.007 & 0.199 & 0.234 \\
\hline External Capsule - Right & $-0.441 *$ & -0.298 & $-0.399 *$ \\
\hline External Capsule - Left & $-0.372 *$ & -0.202 & $-0.407 *$ \\
\hline Fornix - Right & -0.111 & -0.074 & -0.287 \\
\hline Fornix - Left & 0.106 & 0.064 & 0.077 \\
\hline Superior Longitudinal Fasciculus - Right & -0.084 & -0.225 & -0.028 \\
\hline Superior Longitudinal Fasciculus - Left ${ }^{\mathrm{b}}$ & -0.059 & -0.035 & 0.049 \\
\hline \multicolumn{4}{|l|}{ Axial Diffusivity } \\
\hline Entire White Matter Skeleton & 0.058 & -0.165 & -0.092 \\
\hline \multicolumn{4}{|l|}{ Radial Diffusivity } \\
\hline Entire White Matter Skeleton ${ }^{\mathrm{b}}$ & -0.253 & -0.282 & -0.124 \\
\hline
\end{tabular}

${ }^{a}$ r-value reported

$* p \leq 0.1$ (trend); $* * p<0.01$

bLog-transformed 